\title{
Belief and Possibility Belief Interval-Valued N-Soft Set and Their Applications in Multi-Attribute Decision-Making Problems
}

\author{
Shahbaz Ali ${ }^{1}$, Muneeba Kousar ${ }^{1}$, Qin Xin ${ }^{2}$, Dragan Pamučar ${ }^{3, *(\mathbb{D}, \text { Muhammad Shazib Hameed }}{ }^{1}$ \\ and Rabia Fayyaz 4
}

Citation: Ali, S.; Kousar, M.; Qin, X.; Pamucar, D.; Hameed, M.S.; Fayyaz, R. Belief and Possibility Belief Interval-Valued N-Soft Set and Their Applications in Multi-Attribute Decision-Making Problems. Entropy 2021, 23, 1498. https://doi.org/ $10.3390 / \mathrm{e} 23111498$

Academic Editor: Diego Oliva

Received: 11 October 2021

Accepted: 9 November 2021

Published: 13 November 2021

Publisher's Note: MDPI stays neutral with regard to jurisdictional claims in published maps and institutional affiliations.

Copyright: (c) 2021 by the authors. Licensee MDPI, Basel, Switzerland. This article is an open access article distributed under the terms and conditions of the Creative Commons Attribution (CC BY) license (https:/ / creativecommons.org/licenses/by/ $4.0 /)$.
1 Department of Mathematics, Khwaja Fareed University of Engineering \& Information Technology, Rahim Yar Khan 64200, Pakistan; shahbaz.ali@kfueit.edu.pk (S.A.); math202701001@kfueit.edu.pk (M.K.); shazib.hameed@kfueit.edu.pk (M.S.H.)

2 Faculty of Science and Technology, University of the Faroe Islands, Vestarabryggja 15, FO 100 Torshavn, Faroe Islands, Denmark; qinx@setur.fo

3 Department of Logistics, Military Academy, University of Defence in Belgrade, 11000 Belgrade, Serbia

4 Department of Mathematics, COMSATS University Islamabad, Islamabad 44000, Pakistan; rabia_fayyaz@comsats.edu.pk

* Correspondence: dragan.pamucar@va.mod.gov.rs

\begin{abstract}
In this research article, we motivate and introduce the concept of possibility belief intervalvalued N-soft sets. It has a great significance for enhancing the performance of decision-making procedures in many theories of uncertainty. The $\mathrm{N}$-soft set theory is arising as an effective mathematical tool for dealing with precision and uncertainties more than the soft set theory. In this regard, we extend the concept of belief interval-valued soft set to possibility belief interval-valued N-soft set (by accumulating possibility and belief interval with $\mathrm{N}$-soft set), and we also explain its practical calculations. To this objective, we defined related theoretical notions, for example, belief interval-valued $\mathrm{N}$-soft set, possibility belief interval-valued $\mathrm{N}$-soft set, their algebraic operations, and examined some of their fundamental properties. Furthermore, we developed two algorithms by using max-AND and min-OR operations of possibility belief interval-valued $\mathrm{N}$-soft set for decision-making problems and also justify its applicability with numerical examples.
\end{abstract}

Keywords: belief interval-valued soft set; belief interval-valued N-soft set; possibility belief intervalvalued $\mathrm{N}$-soft set; algorithms and applications for decision-making

\section{Introduction}

In real life, the limitation of precise research is progressively being recognized in various fields such as economics, social sciences, medical sciences, computer sciences, physical sciences, environmental sciences, management sciences, and engineering. It is familiar that the real world is full of vagueness, imprecision, and uncertainty, so research on these areas is of great significance. The solutions to such problems engaged the use of mathematical principles on the basis of imprecision and uncertainty. This article expands the scope of applications of one of the theories that can be used to deal with these attributes or characteristics, namely soft set theory.

In this unrealistic environment, there are many problems related to uncertainty [1-4]. However, the maximum mathematical tools that are in existence are crisp [5]. Numerous theories have been introduced to explore uncertainty in an efficient way. For instance, Bayesian network [6], evidence theory [7-9], fuzzy set theory [10,11], intuitionistic fuzzy set (IFS) [12,13], and gray prediction model [14]. Meanwhile, numerous properties of these theories have also been studied broadly [14]. In [15], Molodtsov indicates that there is a difficulty in the fuzzy set and intuitionistic fuzzy set theory, that is, the level of the membership defined by the individual regarded depends on the knowledge received by 
the individual, in consequence, vulnerable to subjective factors. Additionally, different attributes in one problem need to be thought about in an integrated manner. A soft set computing model was developed by Molodtsov [15] to address these restrictions. A problem considering multiple attributes is a virtue of the soft set theory and it has very good potential to solve problems and plays a very significant role in various fields [16,17]. Therefore, for soft set theory, many researchers are introducing methods and operations for it. For instance, the fuzzy soft set theory is an extension of the soft set proposed by $\mathrm{Xu}$ [18].

By accumulating soft set theory with the fuzzy set theory, Maji [19] introduced the notation of FSS (fuzzy soft set), and this theory was used to address decision-making problems. Generalized fuzzy soft sets were proposed by Majumdar and Samanta [20]; their properties were studied and used to solve problems of uncertainty. Maji [21,22] also introduced intuitionistic fuzzy soft sets by integrating IFS with the soft set. Dinda [23] introduced the generalized intuitionistic fuzzy soft sets, belief interval-valued soft sets [24], generalized belief interval-valued soft sets [25], interval-valued intuitionistic fuzzy soft sets [26], interval-valued picture fuzzy soft sets [27], interval-valued neutrosophic soft sets [28], and generalized picture fuzzy soft sets [29]. Further, there are many extension models of the soft set theory rapidly developed; for instance, possibility fuzzy soft set [30], possibility m-polar fuzzy soft sets [31], possibility Pythagorean fuzzy soft sets [32], possibility neutrosophic soft sets [33], possibility multi-fuzzy soft sets [34], and possibility belief interval-valued soft sets [35].

The belief theory was proposed by Dempster and Shafer $[35,36]$. This theory has been applied in various fields. For instance, uncertainty modeling [37], uncertainty reasoning [14,38,39], decision-making [40,41], information fusion [42,43], and other fields [44]. Fatimah [45] extended the soft set model under a non-binary evaluation environment and introduced the concept of N-soft set (NSS) and explained the significance of ordered grades in the practical problems. Furthermore, they also developed decision-making procedures for the N-soft set. Later on, Akram [46] proposed a novel hybrid model known as hesitant $\mathrm{N}$-soft set (HNSS) by accumulating hesitancy and N-soft set. Meanwhile, in [47], they also introduced the concept of fuzzy N-soft set (FNSS) by accumulating a fuzzy set with an $\mathrm{N}$-soft set. Many problems related to decision-making are discussed by using different kinds of environments in [48-58]. The developed model gives a more flexible decisionmaking method for dealing with uncertainties referring to which specific level is allocated to objects in the parameterizations by attributes.

In this article, we present the concept of a possibility belief interval-valued $\mathrm{N}$-soft set, which can be viewable as a possibility belief interval-valued N-soft model. In Section 2, we review the basic idea concerning the Dempster-Shafer theory and in addition, soft set, belief interval-valued soft set (BIVSS) and N-soft set are briefly reminded of with examples. In Section 3, we propose the model of the belief interval-valued N-soft set (BIVNSS). In Section 4, we discuss some algebraic operations (for instance, restricted intersection, restricted union, extended intersection, extended union, complement, top complement, bottom complement, max-AND, and min-OR) on the belief interval-valued $\mathrm{N}$-soft set and many fundamental properties of these operations are introduced. In Section 5, we proposed the model of possibility belief interval-valued N-soft set (PBIVNSS). In Section 6, we introduce many algebraic operations (for example, restricted intersection, restricted union, extended intersection, extended union, complement, top complement, bottom complement, max-AND, and min-OR) on possibility belief interval-valued $\mathrm{N}$-soft set, and various fundamental properties on these operations are also discussed. In Section 7, we develop algorithms on max-AND and min-OR operations of possibility belief intervalvalued N-soft sets for decision making. Then in Section 8, we present the applications on decision-making problems that yield the optimum solution. While in Section 9, we conclude the article. 


\section{Preliminaries}

In this section, a short review of basic definitions and relevant theories are given, which we used to develop the methods introduced in this paper. There are several problems related to uncertainty in this real-life [59-61]. The Dempster-Shafer theory has been broadly used in dealing with the uncertain problems $[62,63]$. The Dempster-Shafer theory is a generalized scheme for demonstrating uncertainty. Dempster proposed a belief measure theory that developed lower and upper probabilities of a system while Shafer provided a thorough belief function explanation.

Definition 1. Let $\mathcal{Y}$ be a finite set of frame of discernment (hypotheses), $2^{\mathcal{Y}}$ be the set of all subsets of $\mathcal{Y}$ and $\hat{\mathcal{Y}} \subseteq \mathcal{Y}$. The belief structure of Dempster-Shafer is associated with a mapping $\mathcal{E}: 2^{\mathcal{Y}} \rightarrow[0,1]$ such that

$$
\mathcal{E}(\Phi)=0, \sum_{\hat{\mathcal{Y}} \in 2^{\mathcal{Y}}} \mathcal{E}(\hat{\mathcal{Y}})=1
$$

is the basic probability assignment function, where $\mathcal{E}(\hat{\mathcal{Y}})$ indicates the belief values of $\mathcal{Y}$. For which subsets of $\mathcal{Y}$ mapping allot non-zero values are known as focal elements [24].

Basic probability assignment has various operations for instance divergence [64], entropy function [65-67], and others [68].

Definition 2 ([35]). The measure of belief function associated with $\mathcal{E}$ is determined as a mapping Bel : $2^{\mathcal{Y}} \rightarrow[0,1]$ such that for any subset $\hat{\mathcal{Z}}$ of $\mathcal{Y}$,

$$
\operatorname{Bel}(\hat{\mathcal{Z}})=\sum_{\Phi \neq \hat{\mathcal{Y}} \subseteq \hat{\mathcal{Z}}} \mathcal{E}(\hat{\mathcal{Y}})
$$

Definition 3 ([35]). The measure of plausibility function associated with $\mathcal{E}$ is determined as a mapping $\mathrm{Pl}: 2^{\mathcal{Y}} \rightarrow[0,1]$ such that for any subset $\hat{\mathcal{Z}}$ of $\mathcal{Y}$,

$$
\operatorname{Pl}(\hat{\mathcal{Z}})=\sum_{\hat{\mathcal{Y}} \cap \hat{\mathcal{Z}} \neq \Phi} \mathcal{E}(\hat{\mathcal{Y}}) .
$$

Obviously, $\operatorname{Bel}(\hat{\mathcal{Z}}) \leq \operatorname{Pl}(\hat{\mathcal{Z}})$. The interval $[\operatorname{Bel}(\hat{\mathcal{Z}}), \operatorname{Pl}(\hat{\mathcal{Z}})]$ is called belief interval (BI) [69].

Definition 4. Let $U$ be the non-empty universal set of objects and $E$ be the set of attributes, for any non-empty set $K \subseteq E$. A pair $(\mathcal{A}, K)$ is called soft set over $U$ if there is a mapping $\mathcal{A}: K \rightarrow 2^{U}$ where $2^{U}$ denotes the set of all subsets of $U$.

Thus, the soft set is a parametric family of the subsets of a universal set. For each $k_{j} \in K$, we can interpret $\mathcal{A}\left(k_{j}\right)$ as a subset of universal set $U$. We can also consider $\mathcal{A}\left(k_{j}\right)$ as a mapping $\mathcal{A}\left(k_{j}\right): U \rightarrow\{0,1\}$ and then $\mathcal{A}\left(k_{j}\right)\left(u_{i}\right)=1$ equivalent to $u_{i} \in \mathcal{A}\left(k_{j}\right)$, otherwise $\mathcal{A}\left(k_{j}\right)\left(u_{i}\right)=$ 0 [45]. Molodtsov considered many examples in [15] to illustrate the soft set.

Definition 5. Let $U$ be the non-empty universal set of objects. Let $B I^{U}$ denote the collection of all belief interval-valued subsets of $U$ and $E$ be the set of attributes, for any non-empty set $K \subseteq E$. A pair $(\mathcal{B}, K)$ is called a belief interval-valued soft set over U (in short BIVSS) if there is a mapping $\mathcal{B}: K \rightarrow B I^{U}$. It is represented as:

$$
\mathcal{B}\left(k_{j}\right)=\left\{\frac{u_{i}}{B I_{\mathcal{B}\left(k_{j}\right)}\left(u_{i}\right)} \mid u_{i} \in U \wedge k_{j} \in K\right\}
$$

where $B I_{\mathcal{B}\left(k_{j}\right)}\left(u_{i}\right)=\left[B e l_{\mathcal{B}\left(k_{j}\right)}\left(u_{i}\right), P l_{\mathcal{B}\left(k_{j}\right)}\left(u_{i}\right)\right], B e l_{\mathcal{B}\left(k_{j}\right)}\left(u_{i}\right) \in[0,1], P l_{\mathcal{B}\left(k_{j}\right)}\left(u_{i}\right) \in[0,1]$, and $0 \leq B e l_{\mathcal{B}\left(k_{j}\right)}\left(u_{i}\right) \leq P l_{\mathcal{B}\left(k_{j}\right)}\left(u_{i}\right) \leq 1$ for all $u_{i} \in U$. 
Example 1. Let $U=\left\{u_{1}, u_{2}, u_{3}, u_{4}, u_{5}\right\}$ be the set of universe, $E=\left\{k_{1}, k_{2}, k_{3}, k_{4}\right\}$ be the set of attributes, and $K \subseteq E$ such that $K=\left\{k_{1}, k_{2}\right\}$. Then BIVSS over $U$ is:

$$
\mathcal{B}\left(k_{1}\right)=\left\{\frac{u_{1}}{[0.5,1.0]}, \frac{u_{2}}{[0.4,0.7]}, \frac{u_{3}}{[0.2,0.4]}\right\}, \mathcal{B}\left(k_{2}\right)=\left\{\frac{u_{1}}{[0.2,0.7]}, \frac{u_{2}}{[0.1,0.6]}, \frac{u_{3}}{[0.3,0.9]}\right\} .
$$

Definition 6. Let $U$ be the non-empty universal set of objects. Let $2^{U}$ denote the set of all subsets of $U$ and let $R=\{0,1,2, \ldots, N-1\}$ be a set of ordered grades where $N \in\{2,3,4, \ldots\}$ and $E$ are the set of attributes, for any non-empty set $K \subseteq E$. A triple $(\mathcal{C}, K, N)$ is called $N$-soft set over $U$ if there is a mapping $\mathcal{C}: K \rightarrow 2^{U \times R}$, with the property that for each $k_{j} \in K$ there exists a unique $\left(u_{i}, r_{i j}\right) \in(U \times R)$ such that $\left(u_{i}, r_{i j}\right) \in \mathcal{C}\left(k_{j}\right), k_{j} \in K, u_{i} \in U$ and $r_{i j} \in R$, where $2^{U \times R}$ is the collection of all soft sets over $U \times R$ [45].

Example 2. Let $U=\left\{u_{1}, u_{2}, u_{3}\right\}$ be the set of students, $E=\left\{k_{1}, k_{2}, k_{3}, k_{4}, k_{5}\right\}$ be the set of attributes evaluations of students by skills, and $K \subseteq E$ such that

$K=\left\{k_{1}=\right.$ communication skills, $k_{3}=$ collaboration skills, $k_{5}=$ critical thinking $\}$ and let $R=\{0,1,2,3,4,5\}$ be the set of grade evaluation. Then, $(\mathcal{C}, K, 6)$ is the 6 -soft set as follows:

$$
\begin{aligned}
& \mathcal{C}\left(k_{1}\right)=\left\{\left(u_{1}, 4\right),\left(u_{2}, 2\right),\left(u_{3}, 3\right)\right\}, \mathcal{C}\left(k_{3}\right)=\left\{\left(u_{1}, 2\right),\left(u_{2}, 1\right),\left(u_{3}, 5\right)\right\}, \text { and } \mathcal{C}\left(k_{5}\right)= \\
& \left\{\left(u_{1}, 5\right),\left(u_{2}, 3\right),\left(u_{3}, 0\right)\right\} . \\
& \quad \text { It can also be represented in tabular form as follows: }
\end{aligned}
$$

\begin{tabular}{cccc}
\hline$(\mathcal{C}, K, 6)$ & $k_{1}$ & $k_{3}$ & $k_{5}$ \\
\hline$u_{1}$ & 4 & 2 & 5 \\
$u_{2}$ & 2 & 1 & 3 \\
$u_{3}$ & 3 & 5 & 0 \\
\hline
\end{tabular}

For illustration, the above table is of a 6 -soft set $(\mathcal{C}, K, 6)$ established on communication skills, collaboration skills, and critical thinking of the students. Where in the top left cell 4 is the ordered grade $\left(r_{11}\right)$ of the student $u_{1}$ with respect to $k_{1}=$ communication skills. Similarly, in the bottom right cell, 0 is the ordered grade $\left(r_{35}\right)$ of the student $u_{3}$ with respect to $k_{5}=$ critical thinking. Here, 0 is the lowest grade; it does not mean that there is no evaluation or incomplete information. There are many examples to illustrate the $\mathrm{N}$-soft set in [45].

\section{Belief Interval-Valued N-Soft Set (BIVNSS)}

In this section, we derive some basic concepts of a new extended model of a belief interval-valued $\mathrm{N}$-soft set with examples from real practice.

Definition 7. Let $U$ be the non-empty universal set of objects and $E$ be the set of attributes, for any non-empty set $K \subseteq E$ and let $B I^{U}$ denote the collection of all belief interval-valued subsets of $U$ and $R=\{0,1,2, \ldots, N-1\}$ be a set of ordered grades where $N \in\{2,3,4, \ldots\}$. A triple $(A, K, N)$ is called a belief interval valued $N$-soft set over $U$ if there is a mapping $A: K \rightarrow B I^{U \times R}$, where $B I^{U \times R}$ is the collection of all belief interval-valued soft sets over $U \times R$. It is represented as:

$$
A\left(k_{j}\right)=\left\{\frac{\left(u_{i}, r_{i j}\right)}{B I_{A\left(k_{j}\right)}\left(u_{i}, r_{i j}\right)} \mid\left(u_{i}, r_{i j}\right) \in(U \times R)\right\}, \forall k_{j} \in K \subseteq E,
$$

where,

$$
B I_{A\left(k_{j}\right)}\left(u_{i}, r_{i j}\right)=\left[\operatorname{Bel}_{A\left(k_{j}\right)}\left(u_{i}, r_{i j}\right), P l_{A\left(k_{j}\right)}\left(u_{i}, r_{i j}\right)\right], \operatorname{Bel}_{A\left(k_{j}\right)}\left(u_{i}, r_{i j}\right) \in[0,1]
$$


and

$$
P l_{A\left(k_{j}\right)}\left(u_{i}, r_{i j}\right) \in[0,1], 0 \leq \operatorname{Bel}_{A\left(k_{j}\right)}\left(u_{i}, r_{i j}\right) \leq P l_{A\left(k_{j}\right)}\left(u_{i}, r_{i j}\right) \leq 1 ; \forall u_{i} \in U \text { and } r_{i j} \in R .
$$

Example 3. Let $U=\left\{u_{1}, u_{2}, u_{3}\right\}$ be the universe of gardens, $R=\{0,1,2,3,4\}$ be the set of grade evaluation, $E=\left\{k_{1}=\right.$ Rose, $k_{2}=$ Tulip, $k_{3}=$ Jasmine, $k_{4}=$ Daffodils $\}$ be the set of attributes (evaluation of gardens by flowers), and $K \subseteq E$ such that $K=\left\{k_{1}, k_{3}, k_{4}\right\}$.

Thus, $(A, K, 5)$ is the belief interval of 5-soft set as follow:

$(A, K, 5)=\left\{\left(k_{1},\left(\frac{\left(u_{1}, 3\right)}{[0.4,0.8]}, \frac{\left(u_{2}, 0\right)}{[0.1,0.7]}, \frac{\left(u_{3}, 4\right)}{[0.5,0.8]}\right)\right),\left(k_{3},\left(\frac{\left(u_{1}, 2\right)}{[0.3,0.5]}, \frac{\left(u_{2}, 4\right)}{[0.8,0.9]}, \frac{\left(u_{3}, 1\right)}{[0.2,0.4]}\right)\right),\left(k_{4},\left(\frac{\left(u_{1}, 1\right)}{[0.7,0.9]}, \frac{\left(u_{2}, 3\right)}{[0.3,0.8]}, \frac{\left(u_{3}, 2\right)}{[0.4,0.7]}\right)\right)\right\}$.

\section{Operations on BIVNSS}

In this section, we discussed some algebraic operations on belief interval-valued $\mathrm{N}$-soft set and their properties.

Definition 8. Let $U$ be the non-empty universal set of objects. Given that $(B, K, M)$ and $(C, L, N)$ are two BIVNSS on $U$, their restricted intersection is defined as:

$$
(D, T, O)=(B, K, M) \cap_{R}(C, L, N),
$$

where $D=B \cap_{R} C, T=K \cap L \neq \Phi$ and $O=\min (M, N)$, i.e., $\forall t_{j} \in T \wedge u_{i}=U$,

$$
\begin{aligned}
\frac{\left(u_{i}, r_{i j}\right)}{B I_{D\left(t_{j}\right)}\left(u_{i}, r_{i j}\right)} \in D\left(t_{j}\right) \Leftrightarrow & r_{i j}=\min \left(r_{i j}^{1}, r_{i j}^{2}\right), \\
& B I_{D\left(t_{j}\right)}\left(u_{i}, r_{i j}\right)=\left[B e l_{D\left(t_{j}\right)}\left(u_{i}, r_{i j}\right), P l_{D\left(t_{j}\right)}\left(u_{i}, r_{i j}\right)\right]
\end{aligned}
$$

where,

$$
\begin{aligned}
{\left[\operatorname{Bel}_{D\left(t_{j}\right)}\left(u_{i}, r_{i j}\right), P l_{D\left(t_{j}\right)}\left(u_{i}, r_{i j}\right)\right]=} & {\left[\min \left(\operatorname{Bel}_{D\left(t_{j}\right)}\left(u_{i}, r_{i j}^{1}\right), B e l_{D\left(t_{j}\right)}\left(u_{i}, r_{i j}^{2}\right)\right),\right.} \\
& \left.\min \left(P l_{D\left(t_{j}\right)}\left(u_{i}, r_{i j}^{1}\right), P l_{D\left(t_{j}\right)}\left(u_{i}, r_{i j}^{2}\right)\right)\right] .
\end{aligned}
$$

If $\frac{\left(u_{i}, r_{i j}^{1}\right)}{\left[B e l_{D\left(t_{j}\right)}\left(u_{i}, r_{i j}^{1}\right), P l_{D\left(t_{j}\right)}\left(u_{i}, r_{i j}^{1}\right)\right]} \in B\left(t_{j}^{1}\right)$ and $\frac{\left(u_{i}, r_{i j}^{2}\right)}{\left[\operatorname{BelD}\left(t_{j}\right)\left(u_{i}, r_{i j}^{2}\right), P l_{D\left(t_{j}\right)}\left(u_{i}, r_{i j}^{2}\right)\right]} \in C\left(t_{j}^{2}\right)$ with $t_{j}^{1} \in$ $K$ and $t_{j}^{2} \in L$.

Definition 9. Let $U$ be the non-empty universal set of objects. Given that $(B, K, M)$ and $(C, L, N)$ are two BIVNSS on $U$, their restricted union is defined as:

$$
(E, T, P)=(B, K, M) \cup_{R}(C, L, N),
$$

where $E=B \cup_{R} C, T=K \cap L$ and $P=\max (M, N)$, i.e., $\forall t_{j} \in T$ and $u_{i}=U$,

$$
\begin{aligned}
\frac{\left(u_{i}, r_{i j}\right)}{B I_{E\left(t_{j}\right)}\left(u_{i}, r_{i j}\right)} \in E\left(t_{j}\right) \Leftrightarrow & r_{i j}=\max \left(r_{i j}^{1}, r_{i j}^{2}\right), \\
& B I_{E\left(t_{j}\right)}\left(u_{i}, r_{i j}\right)=\left[\operatorname{Bel}_{E\left(t_{j}\right)}\left(u_{i}, r_{i j}\right), P l_{E\left(t_{j}\right)}\left(u_{i}, r_{i j}\right)\right],
\end{aligned}
$$


where,

$$
\begin{aligned}
& {\left[\operatorname{Bel}_{E\left(t_{j}\right)}\left(u_{i}, r_{i j}\right), P l_{E\left(t_{j}\right)}\left(u_{i}, r_{i j}\right)\right]=\left[\max \left(\operatorname{Bel}_{E\left(t_{j}\right)}\left(u_{i}, r_{i j}^{1}\right), \operatorname{Bel}_{E\left(t_{j}\right)}\left(u_{i}, r_{i j}^{2}\right)\right),\right.} \\
& \left.\max \left(P l_{E\left(t_{j}\right)}\left(u_{i}, r_{i j}^{1}\right), P l_{E\left(t_{j}\right)}\left(u_{i}, r_{i j}^{2}\right)\right)\right] . \\
& \text { If } \frac{\left(u_{i}, r_{i j}^{1}\right)}{\left[\operatorname{Bel}_{E\left(t_{j}\right)}\left(u_{i}, r_{i j}^{1}\right), P l_{E\left(t_{j}\right)}\left(u_{i}, r_{i j}\right)\right]} \in B\left(t_{j}^{1}\right) \text { and } \frac{\left(u_{i}, r_{i j}^{2}\right)}{\left[\operatorname{BelE}\left(t_{j}\right)\left(u_{i}, r_{i j}^{2}\right), P l_{E\left(t_{j}\right)}\left(u_{i}, r_{i j}^{2}\right)\right]} \in C\left(t_{j}^{2}\right) \text { with } t_{j}^{1} \in
\end{aligned}
$$
$K$ and $t_{j}^{2} \in L$.

Definition 10. Let $U$ be the non-empty universal set of objects. Given that $(B, K, M)$ and $(C, L, N)$ are two BIVNSS on $U$, their extended intersection is defined as:

$$
(F, S, P)=(B, K, M) \cap_{E}(C, L, N),
$$

where $F=B \cap_{E} C, S=K \cup L$ and $P=\max (M, N)$, i.e., $\forall s_{j} \in S, u_{i}=U$ with $s_{j}^{1} \in$ $K$, and $s_{j}^{2} \in L$,

$$
F\left(s_{j}\right)= \begin{cases}B\left(s_{j}^{1}\right) & , \text { if } s_{j} \in K-L, \\ C\left(s_{j}^{2}\right) & , \text { if } s_{j} \in L-K, \\ B\left(s_{j}^{1}\right) \cap_{R} C\left(s_{j}^{2}\right) & , \text { if } s_{j} \in K \cap L .\end{cases}
$$

Definition 11. Let $U$ be the non-empty universal set of objects. Given that $(B, K, M)$ and $(C, L, N)$ are two BIVNSS on $U$, their extended union is defined as:

$$
(H, S, P)=(B, K, M) \cup_{E}(C, L, N),
$$

where $H=B \cup_{E} C, S=K \cup L$ and $P=\max (M, N)$, i.e., $\forall s_{j} \in S, u_{i}=U$ with $s_{j}^{1} \in$ K and $s_{j}^{2} \in L$,

$$
H\left(s_{j}\right)= \begin{cases}B\left(s_{j}^{1}\right) & , \text { if } s_{j} \in K-L, \\ C\left(s_{j}^{2}\right) & , \text { if } s_{j} \in L-K, \\ B\left(s_{j}^{1}\right) \cup_{R} C\left(s_{j}^{2}\right) & , \text { if } s_{j} \in L \cap K .\end{cases}
$$

Example 4. Let $U=\left\{u_{1}, u_{2}, u_{3}\right\}$ be the set of Covid-19 patients, $E=\left\{k_{1}=\right.$ tiredness, $k_{2}=$ skin rashes, $k_{3}=$ dry cough, $k_{4}=$ shortness of breath $\}$ be the set of attributes and $K, L \subseteq E$ such that $K=\left\{k_{1}, k_{3}, k_{4}\right\}, L=\left\{k_{1}, k_{3}\right\}$. The BIVNSS are defined as follows:

$$
\begin{aligned}
&(B, K, 5)=\left\{\left(k_{1},\left(\frac{\left(u_{1}, 1\right)}{[0.3,0.8]}, \frac{\left(u_{2}, 4\right)}{[0.1,0.5]}, \frac{\left(u_{3}, 2\right)}{[0.8,0.9]}\right)\right),\left(k_{3},\left(\frac{\left(u_{1}, 3\right)}{[0.1,0.5]}, \frac{\left(u_{2}, 1\right)}{[0.3,0.4]}, \frac{\left(u_{3}, 4\right)}{[0.5,0.9]}\right)\right),\right. \\
&\left.\left(k_{4},\left(\frac{\left(u_{1}, 2\right)}{[0.3,0.7]}, \frac{\left(u_{2}, 4\right)}{[0.4,0.6]}, \frac{\left(u_{3}, 3\right)}{[0.3,0.8]}\right)\right)\right\} . \\
&(C, L, 4)=\left\{\left(k_{1},\left(\frac{\left(u_{1}, 0\right)}{[0.3,0.7]}, \frac{\left(u_{2}, 1\right)}{[0.1,0.4]}, \frac{\left(u_{3}, 3\right)}{[0.7,0.8]}\right)\right),\left(k_{3},\left(\frac{\left(u_{1}, 2\right)}{[0.5,0.8]}, \frac{\left(u_{2}, 2\right)}{[0.4,0.9]}, \frac{\left(u_{3}, 0\right)}{[0.7,0.8]}\right)\right)\right\} .
\end{aligned}
$$

Then their restricted intersection is:

$$
(D, T, 4)=\left\{\left(k_{1},\left(\frac{\left(u_{1}, 0\right)}{[0.3,0.7]}, \frac{\left(u_{2}, 1\right)}{[0.1,0.4]}, \frac{\left(u_{3}, 2\right)}{[0.7,0.8]}\right)\right),\left(k_{3},\left(\frac{\left(u_{1}, 2\right)}{[0.1,0.5]}, \frac{\left(u_{2}, 1\right)}{[0.3,0.4]}, \frac{\left(u_{3}, 0\right)}{[0.5,0.8]}\right)\right)\right\} .
$$

their restricted union is:

$$
(E, T, 5)=\left\{\left(k_{1},\left(\frac{\left(u_{1}, 1\right)}{[0.3,0.8]}, \frac{\left(u_{2}, 4\right)}{[0.1,0.5]}, \frac{\left(u_{3}, 3\right)}{[0.8,0.9]}\right)\right),\left(k_{3},\left(\frac{\left(u_{1}, 3\right)}{[0.5,0.8]}, \frac{\left(u_{2}, 2\right)}{[0.4,0.9]}, \frac{\left(u_{3}, 4\right)}{[0.7,0.9]}\right)\right)\right\} .
$$


their extended intersection is:

$$
\begin{aligned}
(F, S, 5)=\{ & \left(k_{1},\left(\frac{\left(u_{1}, 0\right)}{[0.3,0.7]}, \frac{\left(u_{2}, 1\right)}{[0.1,0.4]}, \frac{\left(u_{3}, 2\right)}{[0.7,0.8]}\right)\right),\left(k_{3},\left(\frac{\left(u_{1}, 2\right)}{[0.1,0.5]}, \frac{\left(u_{2}, 1\right)}{[0.3,0.4]}, \frac{\left(u_{3}, 0\right)}{[0.5,0.8]}\right)\right), \\
& \left.\left(k_{4},\left(\frac{\left(u_{1}, 2\right)}{[0.3,0.7]}, \frac{\left(u_{2}, 4\right)}{[0.4,0.6]}, \frac{\left(u_{3}, 3\right)}{[0.3,0.8]}\right)\right)\right\} .
\end{aligned}
$$

their extended union is:

$$
\begin{aligned}
(H, S, 5)=\{ & \left(k_{1},\left(\frac{\left(u_{1}, 1\right)}{[0.3,0.8]}, \frac{\left(u_{2}, 4\right)}{[0.1,0.5]}, \frac{\left(u_{3}, 3\right)}{[0.8,0.9]}\right)\right),\left(k_{3},\left(\frac{\left(u_{1}, 3\right)}{[0.5,0.8]}, \frac{\left(u_{2}, 2\right)}{[0.4,0.9]}, \frac{\left(u_{3}, 4\right)}{[0.7,0.9]}\right)\right), \\
& \left.\left(k_{4},\left(\frac{\left(u_{1}, 2\right)}{[0.3,0.7]}, \frac{\left(u_{2}, 4\right)}{[0.4,0.6]}, \frac{\left(u_{3}, 3\right)}{[0.3,0.8]}\right)\right)\right\} .
\end{aligned}
$$

Definition 12. Let $(A, K, N)$ be a BIVNSS on a non-empty universe $U$. Then a weak belief interval-valued complement is denoted by $\left(A^{c}, K, N\right)$ where $A^{c}\left(k_{j}\right) \cap A\left(k_{j}\right)=\Phi ; \forall k_{j} \in K$ and $A^{c}\left(k_{j}\right)$ is defined as:

$$
A^{c}\left(k_{j}\right)=\left\{\frac{\left(u_{i}, r_{i j}\right)}{B I_{A^{c}\left(k_{j}\right)}\left(u_{i}, r_{i j}\right)} \mid\left(u_{i}, r_{i j}\right) \in U \times R\right\} .
$$

where, $B I_{A^{c}\left(k_{j}\right)}\left(u_{i}, r_{i j}\right)=\left[1-P l_{A\left(k_{j}\right)}\left(u_{i}, r_{i j}\right), 1-B e l_{A\left(k_{j}\right)}\left(u_{i}, r_{i j}\right)\right]$.

Definition 13. For any BIVNSS $(A, K, N)$ on $U$. The bottom weak belief interval-valued complement $\left(A^{<}, K, N\right)$ of $(A, K, N)$ is defined as: $\left(A^{<}, K, N\right)=A\left(k_{j}\right)=$

$$
\left\{\begin{array}{cl}
\frac{\left(u_{i}, 0\right)}{\left[1-P l_{A\left(k_{j}\right)}\left(u_{i}, r_{i j}\right), 1-B e l_{A\left(k_{j}\right)}\left(u_{i}, r_{i j}\right)\right]} & , \text { if } r_{i j}>0, \\
\frac{\left(u_{i}, N-1\right)}{\left[1-P l_{A\left(k_{j}\right)}\left(u_{i}, r_{i j}\right), 1-B e l_{A\left(k_{j}\right)}\left(u_{i}, r_{i j}\right)\right]} & , \text { if } r_{i j}=0 .
\end{array}\right.
$$

Definition 14. For any BIVNSS $(A, K, N)$ on $U$. The top weak belief interval-valued complement $\left(A^{>}, K, N\right)$ of $(A, K, N)$ is defined as: $\left(A^{>}, K, N\right)=A\left(k_{j}\right)=$

$$
\left\{\begin{array}{cl}
\frac{\left(u_{i}, N-1\right)}{\left[1-P l_{A\left(k_{j}\right)}\left(u_{i}, r_{i j}\right), 1-B e l_{A\left(k_{j}\right)}\left(u_{i}, r_{i j}\right)\right]} & , \text { if } r_{i j}<N-1, \\
\frac{\left(u_{i}, 0\right)}{\left[1-P l_{A\left(k_{j}\right)}\left(u_{i}, r_{i j}\right), 1-B e l_{A\left(k_{j}\right)}\left(u_{i}, r_{i j}\right)\right]} & , \text { if } r_{i j}=N-1 .
\end{array}\right.
$$

Example 5. Consider $(A, K, 5)$ as described in Example 3 then its weak belief interval-valued complement is:

$$
\begin{aligned}
&\left(A^{c}, K, 5\right)=\left\{\left(k_{1},\left(\frac{\left(u_{1}, 3\right)}{[0.2,0.6]}, \frac{\left(u_{2}, 0\right)}{[0.3,0.9]}, \frac{u_{3}, 4}{[0.2,0.5]}\right)\right),\left(k_{3},\left(\frac{\left(u_{1}, 2\right)}{[0.5,0.7]}, \frac{\left(u_{2}, 4\right)}{[0.1,0.2]}, \frac{u_{3}, 1}{[0.6,0.8]}\right)\right),\right. \\
&\left.\left(k_{4},\left(\frac{\left(u_{1}, 1\right)}{[0.1,0.3]}, \frac{\left(u_{2}, 3\right)}{[0.2,0.7]}, \frac{u_{3}, 2}{[0.3,0.6]}\right)\right)\right\} .
\end{aligned}
$$

its bottom weak belief interval valued complement is:

$$
\begin{aligned}
\left(A^{<}, K, 5\right)=\{ & \left(k_{1},\left(\frac{\left(u_{1}, 0\right)}{[0.2,0.6]}, \frac{\left(u_{2}, 4\right)}{[0.3,0.9]}, \frac{\left(u_{3}, 0\right)}{[0.2,0.5]}\right)\right),\left(k_{3},\left(\frac{\left(u_{1}, 0\right)}{[0.5,0.7]}, \frac{\left(u_{2}, 0\right)}{[0.1,0.2]}, \frac{\left(u_{3}, 0\right)}{[0.6,0.8]}\right)\right), \\
& \left.\left(k_{4},\left(\frac{\left(u_{1}, 0\right)}{[0.1,0.3]}, \frac{\left(u_{2}, 0\right)}{[0.2,0.7]}, \frac{\left(u_{3}, 0\right)}{[0.3,0.6]}\right)\right)\right\} .
\end{aligned}
$$


its top weak belief interval-valued complement is:

$$
\begin{aligned}
\left(A^{>}, K, 5\right)=\{ & \left(k_{1},\left(\frac{\left(u_{1}, 4\right)}{[0.2,0.6]}, \frac{\left(u_{2}, 4\right)}{[0.3,0.9]}, \frac{\left(u_{3}, 0\right)}{[0.2,0.5]}\right)\right),\left(k_{3},\left(\frac{\left(u_{1}, 4\right)}{[0.5,0.7]}, \frac{\left(u_{2}, 0\right)}{[0.1,0.2]}, \frac{\left(u_{3}, 4\right)}{[0.6,0.8]}\right)\right), \\
& \left.\left(k_{4},\left(\frac{\left(u_{1}, 4\right)}{[0.1,0.3]}, \frac{\left(u_{2}, 4\right)}{[0.2,0.7]}, \frac{\left(u_{3}, 4\right)}{[0.3,0.6]}\right)\right)\right\} .
\end{aligned}
$$

Definition 15. Soft max-AND operation of two BIVNSS $(B, K, M)$ and $(C, L, N)$ (where $B$ : $K \rightarrow B I^{U \times R}$ and $\left.C: L \rightarrow B I^{U \times R}\right)$ defined as:

$$
(B, K, M) \wedge(C, L, N)=(G, K \times L, P),
$$

where, $G: K \times L \rightarrow B I^{U \times R} ; \forall\left(k_{s^{\prime}}, l_{t^{\prime}}\right) \in(K \times L), s^{\prime}, t^{\prime} \in \Lambda$, and $P=\max (M, N)$,

$$
\begin{aligned}
G\left(k_{s^{\prime}}, l_{t^{\prime}}\right) \in & \frac{\left(u_{i}, r_{i\left(s^{\prime}, t^{\prime}\right)}\right)}{B I_{G\left(k_{s^{\prime}}, l_{t^{\prime}}\right)}\left(u_{i}, r_{i\left(s^{\prime}, t^{\prime}\right)}\right)} \Leftrightarrow r_{i\left(s^{\prime}, t^{\prime}\right)}=\max \left(r_{i\left(s^{\prime}, t^{\prime}\right)}^{1}, r_{i\left(s^{\prime}, t^{\prime}\right)}^{2}\right), \\
& B I_{G\left(k_{s^{\prime}}, l_{t^{\prime}}\right)}\left(u_{i}, r_{i\left(s^{\prime}, t^{\prime}\right)}\right)=\left[B e l_{G\left(k_{s^{\prime}}, l_{t^{\prime}}\right)}\left(u_{i}, r_{i\left(s^{\prime}, t^{\prime}\right)}\right), P l_{G\left(k_{s^{\prime}}, l_{t^{\prime}}\right)}\left(u_{i}, r_{i\left(s^{\prime}, t^{\prime}\right)}\right)\right] .
\end{aligned}
$$

where, $\left[\operatorname{Bel}_{G\left(k_{s^{\prime}}, l_{t^{\prime}}\right)}\left(u_{i}, r_{i\left(s^{\prime}, t^{\prime}\right)}\right), P l_{G\left(k_{s^{\prime}}, l_{t^{\prime}}\right)}\left(u_{i}, r_{i\left(s^{\prime}, t^{\prime}\right)}\right)\right]=$

$$
\begin{aligned}
& {\left[\frac{2}{3}\left(\operatorname{Bel}_{B\left(k_{s^{\prime}}\right)}\left(u_{i}, r_{i\left(s^{\prime}, t^{\prime}\right)}\right)+\operatorname{Bel}_{C\left(l_{t^{\prime}}\right)}\left(u_{i}, r_{i\left(s^{\prime}, t^{\prime}\right)}\right)\right)-\right.} \\
& \frac{1}{3} \max \left\{\operatorname{Bel}_{B\left(k_{s^{\prime}}\right)}\left(u_{i}, r_{i\left(s^{\prime}, t^{\prime}\right)}\right), \operatorname{Bel}_{C\left(l_{t^{\prime}}\right)}\left(u_{i}, r_{i\left(s^{\prime}, t^{\prime}\right)}\right)\right\}, \\
& \frac{2}{3}\left(P l_{B\left(k_{s^{\prime}}\right)}\left(u_{i}, r_{i\left(s^{\prime}, t^{\prime}\right)}\right)+P l_{C\left(l_{t^{\prime}}\right)}\left(u_{i}, r_{i\left(s^{\prime}, t^{\prime}\right)}\right)\right)- \\
& \left.\frac{1}{3} \max \left\{P l_{B\left(k_{s^{\prime}}\right)}\left(u_{i}, r_{i\left(s^{\prime}, t^{\prime}\right)}\right), P l_{C\left(l_{t^{\prime}}\right)}\left(u_{i}, r_{i\left(s^{\prime}, t^{\prime}\right)}\right)\right\}\right]
\end{aligned}
$$

with $\left(u_{i}, r_{i\left(s^{\prime}, t^{\prime}\right)}^{1}\right) \in B(K)$ and $\left(u_{i}, r_{i\left(s^{\prime}, t^{\prime}\right)}^{2}\right) \in C(L)$.

Definition 16. Soft min-OR operation of two BIVNSS $(B, K, M)$ and $(C, L, N)$ (where $B: K \rightarrow$ $B I^{U \times R}$ and $\left.C: L \rightarrow B I^{U \times R}\right)$ defined as:

$$
(B, K, M) \vee(C, L, N)=(Q, K \times L, O),
$$

where, $Q: K \times L \rightarrow B I^{U \times R} ; \forall\left(k_{s^{\prime}}, l_{t^{\prime}}\right) \in(K \times L), s^{\prime}, t^{\prime} \in \Lambda$, and $O=\min (M, N)$

$$
\begin{aligned}
Q\left(k_{s^{\prime}}, l_{t^{\prime}}\right) \in & \frac{\left(u_{i}, r_{i\left(s^{\prime}, t^{\prime}\right)}\right)}{B I_{Q\left(k_{s^{\prime}}, l_{t^{\prime}}\right)}\left(u_{i}, r_{i\left(s^{\prime}, t^{\prime}\right)}\right)} \Leftrightarrow r_{i\left(s^{\prime}, t^{\prime}\right)}=\min \left(r_{i\left(s^{\prime}, t^{\prime}\right)}^{1}, r_{i\left(s^{\prime}, t^{\prime}\right)}^{2}\right), \\
B I_{Q\left(k_{s^{\prime}}, l_{t^{\prime}}\right)}\left(u_{i}, r_{i\left(s^{\prime}, t^{\prime}\right)}\right) & =\left[B e l_{Q\left(k_{s^{\prime}}, l_{t^{\prime}}\right)}\left(u_{i}, r_{i\left(s^{\prime}, t^{\prime}\right)}\right), P l_{Q\left(k_{s^{\prime}}, l_{t^{\prime}}\right)}\left(u_{i}, r_{i\left(s^{\prime}, t^{\prime}\right)}\right)\right] .
\end{aligned}
$$

where,

$$
\begin{aligned}
{\left[\operatorname{Bel}_{Q\left(k_{s^{\prime}}, l_{t^{\prime}}\right)}\left(u_{i}, r_{i\left(s^{\prime}, t^{\prime}\right)}\right), P l_{Q\left(k_{s^{\prime}}, l_{t^{\prime}}\right)}\right.} & \left.\left(u_{i}, r_{i\left(s^{\prime}, t^{\prime}\right)}\right)\right]= \\
& {\left[\frac{2}{3}\left(B e l_{B\left(k_{s^{\prime}}\right)}\left(u_{i}, r_{i\left(s^{\prime}, t^{\prime}\right)}\right)+\operatorname{Bel}_{C\left(l_{t^{\prime}}\right)}\left(u_{i}, r_{i\left(s^{\prime}, t^{\prime}\right)}\right)\right)-\right.} \\
& \frac{1}{3} \min \left\{B e l_{B\left(k_{s^{\prime}}\right)}\left(u_{i}, r_{i\left(s^{\prime}, t^{\prime}\right)}\right), B e l_{C\left(l_{t^{\prime}}\right)}\left(u_{i}, r_{i\left(s^{\prime}, t^{\prime}\right)}\right)\right\}, \\
& \frac{2}{3}\left(P l_{B\left(k_{s^{\prime}}\right)}\left(u_{i}, r_{i\left(s^{\prime}, t^{\prime}\right)}\right)+P l_{C\left(l_{t^{\prime}}\right)}\left(u_{i}, r_{i\left(s^{\prime}, t^{\prime}\right)}\right)\right)- \\
& \left.\frac{1}{3} \min \left\{P l_{B\left(k_{s^{\prime}}\right)}\left(u_{i}, r_{i\left(s^{\prime}, t^{\prime}\right)}\right), P l_{C\left(l_{t^{\prime}}\right)}\left(u_{i}, r_{i\left(s^{\prime}, t^{\prime}\right)}\right)\right\}\right]
\end{aligned}
$$


with $\left(u_{i}, r_{i\left(s^{\prime}, t^{\prime}\right)}^{1}\right) \in B(K)$ and $\left(u_{i}, r_{i\left(s^{\prime}, t^{\prime}\right)}^{2}\right) \in C(L)$.

Example 6. Consider $(B, K, 5)$ and $(C, L, 4)$ as described in Example 4, then their soft maxAND is:

$$
\begin{aligned}
(G, K \times L, 5)=\{ & \left(\left(k_{1}, k_{1}\right),\left(\frac{\left(u_{1}, 1\right)}{[0.30,0.73]}, \frac{\left(u_{2}, 40\right)}{[0.10,0.43]}, \frac{\left(u_{3}, 3\right)}{[0.73,0.83]}\right)\right), \\
& \left(\left(k_{1}, k_{3}\right),\left(\frac{\left(u_{1}, 2\right)}{[0.37,0.80]}, \frac{\left(u_{2}, 4\right)}{[0.20,0.63]}, \frac{\left(u_{3}, 2\right)}{[0.73,0.83]}\right)\right), \\
& \left(\left(k_{4}, k_{1}\right),\left(\frac{\left(u_{1}, 2\right)}{[0.30,0.70]}, \frac{\left(u_{2}, 4\right)}{[0.20,0.47]}, \frac{\left(u_{3}, 3\right)}{[0.43,0.80]}\right)\right), \\
& \left(\left(k_{4}, k_{3}\right),\left(\frac{\left(u_{1}, 2\right)}{[0.37,0.73]}, \frac{\left(u_{2}, 4\right)}{[0.40,0.70]}, \frac{\left(u_{3}, 3\right)}{[0.43,0.80]}\right)\right), \\
& \left(\left(k_{3}, k_{1}\right),\left(\frac{\left(u_{1}, 3\right)}{[0.17,0.57]}, \frac{\left(u_{2}, 1\right)}{[0.17,0.40]}, \frac{\left(u_{3}, 4\right)}{[0.57,0.83]}\right)\right), \\
& \left.\left(\left(k_{3}, k_{3}\right),\left(\frac{\left(u_{1}, 3\right)}{[0.23,0.60]}, \frac{\left(u_{2}, 2\right)}{[0.30,0.57]}, \frac{\left(u_{3}, 4\right)}{[0.57,0.83]}\right)\right)\right\} .
\end{aligned}
$$

their soft min-OR is:

$$
\begin{aligned}
(Q, K \times L, 4)=\{ & \left(\left(k_{1}, k_{1}\right),\left(\frac{\left(u_{1}, 0\right)}{[0.30,0.76]}, \frac{\left(u_{2}, 1\right)}{[0.10,0.46]}, \frac{\left(u_{3}, 2\right)}{[0.76,0.86]}\right)\right), \\
& \left(\left(k_{1}, k_{3}\right),\left(\frac{\left(u_{1}, 1\right)}{[0.43,0.80]}, \frac{\left(u_{2}, 2\right)}{[0.30,0.76]}, \frac{\left(u_{3}, 0\right)}{[0.76,0.86]}\right)\right), \\
& \left(\left(k_{4}, k_{1}\right),\left(\frac{\left(u_{1}, 0\right)}{[0.30,0.70]}, \frac{\left(u_{2}, 1\right)}{[0.30,0.53]}, \frac{\left(u_{3}, 3\right)}{[0.56,0.80]}\right)\right), \\
& \left(\left(k_{4}, k_{3}\right),\left(\frac{\left(u_{1}, 2\right)}{[0.43,0.76]}, \frac{\left(u_{2}, 2\right)}{[0.40,0.80]}, \frac{\left(u_{3}, 0\right)}{[0.56,0.80]}\right)\right), \\
& \left(\left(k_{3}, k_{1}\right),\left(\frac{\left(u_{1}, 0\right)}{[0.23,0.63]}, \frac{\left(u_{2}, 1\right)}{[0.23,0.40]}, \frac{\left(u_{3}, 3\right)}{[0.63,0.86]}\right)\right), \\
& \left.\left(\left(k_{3}, k_{3}\right),\left(\frac{\left(u_{1}, 2\right)}{[0.36,0.70]}, \frac{\left(u_{2}, 1\right)}{[0.36,0.73]}, \frac{\left(u_{3}, 0\right)}{[0.63,0.86]}\right)\right)\right\} .
\end{aligned}
$$

Proposition 1. Given that $(B, K, M),(C, L, N)$, and $(D, T, O)$ are any three BIVNSS on $U$, then the commutative and associative properties are held:
(1) $(B, K, M) \cap_{E}(B, K, M)=(B, K, M)$,
(2) $(B, K, M) \cap_{R}(B, K, M)=(B, K, M)$,
(3) $(B, K, M) \cup_{E}(B, K, M)=(B, K, M)$,
(4) $(B, K, M) \cup_{R}(B, K, M)=(B, K, M)$,
(5) $(B, K, M) \cap_{E}(C, L, N)=(C, L, N) \cap_{E}(B, K, M)$,
(6) $(B, K, M) \cap_{R}(C, L, N)=(C, L, N) \cap_{R}(B, K, M)$,
(7) $(B, K, M) \cup_{E}(C, L, N)=(C, L, N) \cup_{E}(B, K, M)$,
(8) $(B, K, M) \cup_{R}(C, L, N)=(C, L, N) \cup_{R}(B, K, M)$,
(9) $(B, K, M) \cap_{E}\left((C, L, N) \cap_{E}(D, T, O)\right)=\left((B, K, M) \cap_{E}(C, L, N)\right) \cap_{E}(D, T, O)$,
(10) $(B, K, M) \cap_{R}\left((C, L, N) \cap_{R}(D, T, O)\right)=\left((B, K, M) \cap_{R}(C, L, N)\right) \cap_{R}(D, T, O)$,
(11) $(B, K, M) \cup_{E}\left((C, L, N) \cup_{E}(D, T, O)\right)=\left((B, K, M) \cup_{E}(C, L, N)\right) \cup_{E}(D, T, O)$,
(12) $(B, K, M) \cup_{R}\left((C, L, N) \cup_{R}(D, T, O)\right)=\left((B, K, M) \cup_{R}(C, L, N)\right) \cup_{R}(D, T, O)$.

Proof. (1)-(8) follows from the definition. (9) Let $(C, L, N)$ and $(D, T, O)$ (where $C: L \rightarrow$ $B I^{U \times R}$ and $\left.D: T \rightarrow B I^{U \times R}\right)$ be two BIVNSS. By the definition of extended intersection we have $(S, V, Z)$ (where $\left.S: V \rightarrow B I^{U \times R}\right)$ such that,

$$
(S, V, Z)=(C, L, N) \cap_{E}(D, T, O),
$$


where $S=C \cap_{E} D, V=L \cup T, Z=\max (N, O) ; \forall v_{j} \in V$ with $v_{j}^{1} \in L$ and $v_{j}^{2} \in T$,

$$
S\left(v_{j}\right)= \begin{cases}C\left(v_{j}^{1}\right) & , \text { if } v_{j} \in L-T, \\ D\left(v_{j}^{2}\right) & , \text { if } v_{j} \in T-L, \\ C\left(v_{j}^{1}\right) \cap_{R} D\left(v_{j}^{2}\right) & , \text { if } v_{j} \in L \cap T .\end{cases}
$$

As, $(B, K, M) \cap_{E}\left((C, L, N) \cap_{E}(D, T, O)\right)=(B, K, M) \cap_{E}(S, V, Z)$. Suppose that $(B, K, M)$ $\cap_{E}(S, V, Z)=(G, W, X)$ such that $G: W \rightarrow B I^{U \times R}$, where $G=B \cap_{E} S, W=K \cup V=$ $K \cup L \cup T, X=\max (M, Z) ; \forall w_{j} \in W$ with $w_{j}^{1} \in K, w_{j}^{2} \in L$ and $w_{j}^{3} \in T$,

$$
G\left(w_{j}\right)= \begin{cases}B\left(w_{j}^{1}\right) & , \text { if } w_{j} \in K-L-T, \\ C\left(w_{j}^{2}\right) & , \text { if } w_{j} \in L-K-T, \\ D\left(w_{j}^{3}\right) & , \text { if } w_{j} \in T-K-L, \\ C\left(w_{j}^{2}\right) \cap_{R} D\left(w_{j}^{3}\right) & , \text { if } w_{j} \in L \cap T-K, \\ B\left(w_{j}^{1}\right) \cap_{R} C\left(w_{j}^{2}\right) & , \text { if } w_{j} \in K \cap L-T, \\ B\left(w_{j}^{1}\right) \cap_{R} D\left(w_{j}^{3}\right) & , \text { if } w_{j} \in K \cap T-L, \\ B\left(w_{j}^{1}\right) \cap_{R} C\left(w_{j}^{2}\right) \cap_{R} D\left(w_{j}^{3}\right) & , \text { if } w_{j} \in K \cap L \cap T .\end{cases}
$$

Again, let $(B, K, M)$ and $(C, L, N)$ (where $B: K \rightarrow B I^{U \times R}$ and $C: L \rightarrow B I^{U \times R}$ ) be two $B I V N S S$. By the definition of extended intersection we have $(F, S, P)$ where $(F: S \rightarrow$ $B I^{U \times R}$ ) such that,

$$
(F, S, P)=(B, K, M) \cap_{E}(C, L, N),
$$

where $F=B \cap_{E} C, S=K \cup L, P=\max (M, N) ; \forall s_{j} \in S$ with $s_{j}^{1} \in K$ and $s_{j}^{2} \in L$,

$$
F\left(s_{j}\right)= \begin{cases}B\left(s_{j}^{1}\right) & , \text { if } s_{j} \in K-L, \\ C\left(s_{j}^{2}\right) & , \text { if } s_{j} \in L-K, \\ B\left(s_{j}^{1}\right) \cap_{R} C\left(s_{j}^{2}\right) & , \text { if } s_{j} \in K \cap L .\end{cases}
$$

As, $\left((B, K, M) \cap_{E}(C, L, N)\right) \cap_{E}(D, T, O)=(F, S, P) \cap_{E}(D, T, O)$. Suppose that $(F, S, P) \cap_{E}$ $(D, T, O)=(I, W, X)$ such that $I: W \rightarrow B I^{U \times R}$, where $I=F \cap_{E} D, W=S \cup T=$ $K \cup L \cup T, X=\max (P, O) ; \forall w_{j} \in W$ with $w_{j}^{1} \in K, w_{j}^{2} \in L$ and $w_{j}^{3} \in T$,

$$
I\left(w_{j}\right)= \begin{cases}B\left(w_{j}^{1}\right) & , \text { if } w_{j} \in K-L-T, \\ C\left(w_{j}^{2}\right) & , \text { if } w_{j} \in L-K-T, \\ D\left(w_{j}^{3}\right) & , \text { if } w_{j} \in T-K-L, \\ C\left(w_{j}^{2}\right) \cap_{R} D\left(w_{j}^{3}\right) & , \text { if } w_{j} \in L \cap T-K, \\ B\left(w_{j}^{1}\right) \cap_{R} C\left(w_{j}^{2}\right) & , \text { if } w_{j} \in K \cap L-T, \\ B\left(w_{j}^{1}\right) \cap_{R} D\left(w_{j}^{3}\right) & , \text { if } w_{j} \in K \cap T-L, \\ B\left(w_{j}^{1}\right) \cap_{R} C\left(w_{j}^{2}\right) \cap_{R} D\left(w_{j}^{3}\right) & , \text { if } w_{j} \in K \cap L \cap T .\end{cases}
$$

Then $G\left(w_{j}\right)=I\left(w_{j}\right), \forall w_{j} \in W$. Hence, (9) is held, (10)-(12) are similar to (9).

Proposition 2. Given that $(B, K, M)$ and $(C, L, N)$ are any two BIVNSS on $U$, then the following results hold:

(1) $\left(\left(B^{c}\right)^{c}, K, M\right)=(B, K, M)$,

(2) $\left(B^{c}, K, M\right) \cup_{E}\left(C^{c}, L, N\right)=\left(\left(B \cap_{E} C\right)^{c},(K \cup L), \max (M, N)\right)$,

(3) $\left(B^{c}, K, M\right) \cap_{E}\left(C^{c}, L, N\right)=\left(\left(B \cup_{E} C\right)^{c},(K \cup L), \max (M, N)\right)$,

(4) $\left(B^{c}, K, M\right) \cup_{R}\left(C^{c}, L, N\right)=\left(\left(B \cap_{R} C\right)^{c},(K \cap L), \max (M, N)\right)$,

(5) $\quad\left(B^{c}, K, M\right) \cap_{R}\left(C^{c}, L, N\right)=\left(\left(B \cup_{R} C\right)^{c},(K \cap L), \min (M, N)\right)$. 
Proof. (1) is straight-forward. We start from (2), let $(B, K, M)$ and $(C, L, N)$ (where $B$ : $\left.K \rightarrow B I^{U \times R} C: L \rightarrow B I^{U \times R}\right)$ be two BIVNSS on $U$. Then by the definition of weak belief interval-valued complement and extended union we have,

$$
\left(B^{c}, K, M\right) \cup_{E}\left(C^{c}, L, N\right)=(J, S, P),
$$

where $J=B^{c} \cup_{E} C^{c}, S=K \cup L, P=\max (M, N) ; \forall s_{j} \in S$ with $s_{j}^{1} \in K$ and $s_{j}^{2} \in L$,

$$
J\left(s_{j}\right)= \begin{cases}B^{c}\left(s_{j}^{1}\right) & , \text { if } s_{j} \in K-L, \\ C^{c}\left(s_{j}^{2}\right) & , \text { if } s_{j} \in L-K, \\ B^{c}\left(s_{j}^{1}\right) \cup_{E} C^{c}\left(s_{j}^{2}\right), & \text { if } s_{j} \in K \cap L .\end{cases}
$$

Again by the definition of extended intersection we have $(F, S, P)=\left(B \cap_{E} C\right), S=$ $K \cup L, P=\max (M, N) ; \forall s_{j} \in S$ with $s_{j}^{1} \in K$ and $s_{j}^{2} \in L$,

$$
F\left(s_{j}\right)= \begin{cases}B\left(s_{j}^{1}\right) & , \text { if } s_{j} \in K-L, \\ C\left(s_{j}^{2}\right) & , \text { if } s_{j} \in L-K, \\ B\left(s_{j}^{1}\right) \cap_{E} C\left(s_{j}^{2}\right) & , \text { if } s_{j} \in K \cap L .\end{cases}
$$

Thus, by the definition of weak complement we have,

$$
F^{c}\left(s_{j}\right)= \begin{cases}B^{c}\left(s_{j}^{1}\right) & , \text { if } s_{j} \in K-L, \\ C^{c}\left(s_{j}^{2}\right) & , \text { if } s_{j} \in L-K, \\ B^{c}\left(s_{j}^{1}\right) \cup_{E} C^{c}\left(s_{j}^{2}\right), & \text { if } s_{j} \in K \cap L .\end{cases}
$$

Then, $J\left(s_{j}\right)=F^{c}\left(s_{j}\right) ; \forall s_{j} \in S$. Hence, (2) holds, and (3)-(5) are similar to (2).

\section{Possibility Belief Interval-Valued N-Soft Set (PBIVNSS)}

In this section, we defined the notion of possibility belief interval-valued $N$-soft set.

Definition 17. Assume that $B I^{U}$ is the set of all belief interval-valued subsets of $U$ and $E$ is the set of attributes, for any non-empty set $K \subseteq E$. The pair $(U, K)$ is called a soft universe, and $R=\{0,1,2, \ldots, N-1\}$ is a set of ordered grades where $N \in\{2,3,4, \ldots\}$. Let $B: K \rightarrow B I^{U \times R}$, and $b$ is a belief interval-valued subsets of $K$, i.e., $b: K \rightarrow B I^{U \times R}$ where $B I^{U \times R}$ is the collection of all belief interval-valued soft sets over $U \times R$. A triple $\left(B_{b}, K, N\right)$ is called a possibility belief interval-valued $N$-soft set over $(U, K)$ if there is a mapping $B_{b}: K \rightarrow B I^{U \times R} \times B I^{U \times R}$. It is represented as:

$$
B_{b}\left(k_{j}\right)\left(u_{i}, r_{i j}\right)=\left\{\left[B e l_{B\left(k_{j}\right)}\left(u_{i}, r_{i j}\right), P l_{B\left(k_{j}\right)}\left(u_{i}, r_{i j}\right)\right],\left[B e l_{b\left(k_{j}\right)}\left(u_{i}, r_{i j}\right), P l_{b\left(k_{j}\right)}\left(u_{i}, r_{i j}\right)\right]\right\}
$$

such that $\left(u_{i}, r_{i j}\right) \in U \times R$ for all $k_{j} \in K \subseteq E$.

Example 7. Let $U=\left\{u_{1}, u_{2}, u_{3}\right\}, R=\{0,1,2,3,4,5,6\}, E=\left\{k_{1}, k_{2}, k_{3}, k_{4}\right\}$ and $K \subseteq E$ such that $K=\left\{k_{1}, k_{2}, k_{3}\right\}$. Then, $\left(B_{b}, K, 7\right)$ is the possibility belief interval of 7 -soft set as follows:

$$
\begin{aligned}
\left(B_{b}, K, 7\right)=\{ & \left(k_{1},\left(\frac{\left(u_{1}, 1\right)}{[0.3,0.8],[0.3,0.7]}, \frac{\left(u_{2}, 5\right)}{[0.3,0.4],[0.1,0.5]}, \frac{\left(u_{3}, 2\right)}{[0.4,0.6],[0.3,0.5]}\right)\right), \\
& \left(k_{2},\left(\frac{\left(u_{1}, 4\right)}{[0.6,0.7],[0.5,0.7]}, \frac{\left(u_{2}, 0\right)}{[0.1,0.8],[0.7,0.9]}, \frac{\left(u_{3}, 6\right)}{[0.6,0.8],[0.4,0.7]}\right)\right), \\
& \left.\left(k_{3},\left(\frac{\left(u_{1}, 6\right)}{[0.1,0.2],[0.4,0.5]}, \frac{\left(u_{2}, 3\right)}{[0.8,0.9],[0.7,0.8]}, \frac{\left(u_{3}, 5\right)}{[0.8,0.9],[0.3,0.6]}\right)\right)\right\} .
\end{aligned}
$$




\section{Operations on PBIVNSS}

In this section, we discussed some algebraic operations on a possibility belief interval valued $\mathrm{N}$-soft set and their fundamental properties.

Definition 18. Let $U$ be the non-empty universal set of objects. Given that $\left(B_{b}, K, M\right)$ and $\left(Y_{y}, L, N\right)$ are two PBIVNSS on $U$, their restricted intersection is defined as:

$$
\left(D_{d}, T, O\right)=\left(B_{b}, K, M\right) \sqcap_{R}\left(Y_{y}, L, N\right),
$$

where $D=B \cap_{R} Y, d=b \cap_{R} y, T=K \cap L \neq \Phi$ and $O=\min (M, N)$, i.e., $\forall t_{j} \in$ $T$ and $u_{i}=U$,

$$
\begin{aligned}
\frac{\left(u_{i}, r_{i j}\right)}{B I_{D\left(t_{j}\right)}\left(u_{i}, r_{i j}\right), B I_{d\left(t_{j}\right)}\left(u_{i}, r_{i j}\right)} \in D_{d}\left(t_{j}\right) & \Leftrightarrow r_{i j}=\min \left(r_{i j}^{1}, r_{i j}^{2}\right), \\
& B I_{D\left(t_{j}\right)}\left(u_{i}, r_{i j}\right)=\left[\operatorname{Bel}_{D\left(t_{j}\right)}\left(u_{i}, r_{i j}\right), P l_{D\left(t_{j}\right)}\left(u_{i}, r_{i j}\right)\right], \\
& B I_{d\left(t_{j}\right)}\left(u_{i}, r_{i j}\right)=\left[\operatorname{Bel}_{d\left(t_{j}\right)}\left(u_{i}, r_{i j}\right), P l_{d\left(t_{j}\right)}\left(u_{i}, r_{i j}\right)\right] .
\end{aligned}
$$

where,

$$
\begin{aligned}
{\left[\operatorname{Bel}_{D\left(t_{j}\right)}\left(u_{i}, r_{i j}\right), P l_{D\left(t_{j}\right)}\left(u_{i}, r_{i j}\right)\right]=} & {\left[\min \left(B e l_{D\left(t_{j}\right)}\left(u_{i}, r_{i j}^{1}\right), B e l_{D\left(t_{j}\right)}\left(u_{i}, r_{i j}^{2}\right)\right),\right.} \\
& \left.\min \left(P l_{D\left(t_{j}\right)}\left(u_{i}, r_{i j}^{1}\right), P l_{D\left(t_{j}\right)}\left(u_{i}, r_{i j}^{2}\right)\right)\right]
\end{aligned}
$$

and

$$
\begin{aligned}
& {\left[\operatorname{Bel}_{d\left(t_{j}\right)}\left(u_{i}, r_{i j}\right), P l_{d\left(t_{j}\right)}\left(u_{i}, r_{i j}\right)\right]=} {\left[\min \left(B e l_{d\left(t_{j}\right)}\left(u_{i}, r_{i j}^{1}\right), B e l_{d\left(t_{j}\right)}\left(u_{i}, r_{i j}^{2}\right)\right),\right.} \\
&\left.\min \left(P l_{d\left(t_{j}\right)}\left(u_{i}, r_{i j}^{1}\right), P l_{d\left(t_{j}\right)}\left(u_{i}, r_{i j}^{2}\right)\right)\right] . \\
& \text { If }\left(\left(u_{i}, r_{i j}^{1}\right),\left[B e l_{D\left(t_{j}\right)}\left(u_{i}, r_{i j}^{1}\right), P l_{D\left(t_{j}\right)}\left(u_{i}, r_{i j}^{1}\right)\right],\left[B e l_{d\left(t_{j}\right)}\left(u_{i}, r_{i j}^{1}\right), P l_{d\left(t_{j}\right)}\left(u_{i}, r_{i j}^{1}\right)\right]\right) \in B_{b}\left(t_{j}^{1}\right) \text { and } \\
&\left(\left(u_{i}, r_{i j}^{2}\right),\left[\operatorname{Bel}_{D\left(t_{j}\right)}\left(u_{i}, r_{i j}^{2}\right), P l_{D\left(t_{j}\right)}\left(u_{i}, r_{i j}^{2}\right)\right],\left[\operatorname{Bel}_{d\left(t_{j}\right)}\left(u_{i}, r_{i j}^{2}\right), P l_{d\left(t_{j}\right)}\left(u_{i}, r_{i j}^{2}\right)\right]\right) \in Y_{y}\left(t_{j}^{2}\right), \text { with } \\
& t_{j}^{1} \in K \text { and } t_{j}^{2} \in L .
\end{aligned}
$$

Definition 19. Let $U$ be the non-empty universal set of objects. Given that $\left(B_{b}, K, M\right)$ and $\left(Y_{y}, L, N\right)$ are two PBIVNSS on $U$, their restricted union is defined as:

$$
\left(E_{e}, T, P\right)=\left(B_{b}, K, M\right) \sqcup_{R}\left(Y_{y}, L, N\right)
$$

where $E=B \cup_{R} Y, e=b \cup_{R} y, T=K \cap L$ and $P=\max (M, N)$, i.e., $\forall t_{j} \in T$ and $u_{i}=U$,

$$
\begin{aligned}
\frac{\left(u_{i}, r_{i j}\right)}{B I_{E\left(t_{j}\right)}\left(u_{i}, r_{i j}\right), B I_{e\left(t_{j}\right)}\left(u_{i}, r_{i j}\right)} \in E_{e}\left(t_{j}\right) \Leftrightarrow r_{i j}=\max \left(r_{i j}^{1}, r_{i j}^{2}\right), \\
\\
B I_{E\left(t_{j}\right)}\left(u_{i}, r_{i j}\right)=\left[\operatorname{Bel}_{E\left(t_{j}\right)}\left(u_{i}, r_{i j}\right), P l_{E\left(t_{j}\right)}\left(u_{i}, r_{i j}\right)\right], \\
B I_{e\left(t_{j}\right)}\left(u_{i}, r_{i j}\right)=\left[\operatorname{Bel}_{e\left(t_{j}\right)}\left(u_{i}, r_{i j}\right), P l_{e\left(t_{j}\right)}\left(u_{i}, r_{i j}\right)\right] .
\end{aligned}
$$


where,

$$
\begin{aligned}
{\left[\operatorname{Bel}_{E\left(t_{j}\right)}\left(u_{i}, r_{i j}\right), P l_{E\left(t_{j}\right)}\left(u_{i}, r_{i j}\right)\right]=} & {\left[\max \left(\operatorname{Bel}_{E\left(t_{j}\right)}\left(u_{i}, r_{i j}^{1}\right), \operatorname{Bel} l_{E\left(t_{j}\right)}\left(u_{i}, r_{i j}^{2}\right)\right),\right.} \\
& \left.m a x\left(P l_{E\left(t_{j}\right)}\left(u_{i}, r_{i j}^{1}\right), P l_{E\left(t_{j}\right)}\left(u_{i}, r_{i j}^{2}\right)\right)\right]
\end{aligned}
$$

and

$$
\begin{aligned}
{\left[\operatorname{Bel}_{e\left(t_{j}\right)}\left(u_{i}, r_{i j}\right), P l_{e\left(t_{j}\right)}\left(u_{i}, r_{i j}\right)\right]=} & {\left[\max \left(\operatorname{Bel}_{e\left(t_{j}\right)}\left(u_{i}, r_{i j}^{1}\right), \operatorname{Bel}_{e\left(t_{j}\right)}\left(u_{i}, r_{i j}^{2}\right)\right),\right.} \\
& \left.\max \left(P l_{e\left(t_{j}\right)}\left(u_{i}, r_{i j}^{1}\right), P l_{e\left(t_{j}\right)}\left(u_{i}, r_{i j}^{2}\right)\right)\right] .
\end{aligned}
$$

If $\left(\left(u_{i}, r_{i j}^{1}\right),\left[B e l_{E\left(t_{j}\right)}\left(u_{i}, r_{i j}^{1}\right), P l_{E\left(t_{j}\right)}\left(u_{i}, r_{i j}^{1}\right)\right],\left[B e l_{e\left(t_{j}\right)}\left(u_{i}, r_{i j}^{1}\right), P l_{e\left(t_{j}\right)}\left(u_{i}, r_{i j}^{1}\right)\right]\right) \in B_{b}\left(t_{j}^{1}\right)$ and $\left(\left(u_{i}, r_{i j}^{2}\right),\left[B e l_{E\left(t_{j}\right)}\left(u_{i}, r_{i j}^{2}\right), P l_{E\left(t_{j}\right)}\left(u_{i}, r_{i j}^{2}\right)\right],\left[B e l_{e\left(t_{j}\right)}\left(u_{i}, r_{i j}^{2}\right), P l_{e\left(t_{j}\right)}\left(u_{i}, r_{i j}^{2}\right)\right]\right) \in Y_{y}\left(t_{j}^{2}\right)$, with $t_{j}^{1} \in K$ and $t_{j}^{2} \in L$.

Definition 20. Let $U$ be the non-empty universal set of objects. Given that $\left(B_{b}, K, M\right)$ and $\left(Y_{y}, L, N\right)$ are two PBIVNSS on $U$, their extended intersection is defined as:

$$
\left(F_{f}, S, P\right)=\left(B_{b}, K, M\right) \sqcap_{E}\left(Y_{y}, L, N\right)
$$

where $F=B \cap_{E} Y, f=b \cap_{E} y, S=K \cup L$ and $P=\max (M, N)$, i.e., $\forall s_{j} \in S, u_{i}=$ $U$ with $s_{j}^{1} \in K$ and $s_{j}^{2} \in L$,

$$
F_{f}\left(s_{j}\right)= \begin{cases}B_{b}\left(s_{j}^{1}\right) & , \text { if } s_{j} \in K-L, \\ Y_{y}\left(s_{j}^{2}\right) & , \text { if } s_{j} \in L-K, \\ B_{b}\left(s_{j}^{1}\right) \sqcap_{R} Y_{y}\left(s_{j}^{2}\right) & , \text { if } s_{j} \in L \cap K .\end{cases}
$$

Definition 21. Let $U$ be the non-empty universal set of objects. Given that $\left(B_{b}, K, M\right)$ and $\left(Y_{y}, L, N\right)$ are two PBIVNSS on $U$, their extended union is defined as:

$$
\left(H_{h}, S, P\right)=\left(B_{b}, K, M\right) \sqcup_{E}\left(Y_{y}, L, N\right)
$$

where $H=B \cup_{E} Y, h=b \cup_{E} y, S=K \cup L$ and $P=\max (M, N)$, i.e., $\forall s_{j} \in S, u_{i}=U, u_{i}=$ $U$ with $s_{j}^{1} \in K$ and $s_{j}^{2} \in L$,

$$
H_{h}\left(s_{j}\right)= \begin{cases}B_{b}\left(s_{j}^{1}\right) & , \text { if } s_{j} \in K-L, \\ Y_{y}\left(s_{j}^{2}\right) & , \text { if } s_{j} \in L-K, \\ B_{b}\left(s_{j}^{1}\right) \sqcup_{R} Y_{y}\left(s_{j}^{2}\right) & , \text { if } s_{j} \in L \cap K .\end{cases}
$$

Example 8. Let $U=\left\{u_{1}, u_{2}, u_{3}\right\}, E=\left\{k_{1}, k_{2}, k_{3}, k_{4}\right\}$ and $K \wedge L \subseteq E$ such that $K=$ $\left\{k_{1}, k_{2}, k_{3}\right\}, L=\left\{k_{2}, k_{4}\right\}$. Then PBIVNSS are defined as follows:

$$
\begin{aligned}
\left(B_{b}, K, 7\right)=\{ & \left(k_{1},\left(\frac{\left(u_{1}, 1\right)}{[0.3,0.8],[0.3,0.7]}, \frac{\left(u_{2}, 5\right)}{[0.3,0.4],[0.1,0.5]}, \frac{\left(u_{3}, 2\right)}{[0.4,0.6],[0.3,0.5]}\right)\right), \\
& \left(k_{2},\left(\frac{\left(u_{1}, 4\right)}{[0.6,0.7],[0.5,0.7]}, \frac{\left(u_{2}, 0\right)}{[0.1,0.8],[0.7,0.9]}, \frac{\left(u_{3}, 6\right)}{[0.6,0.8],[0.4,0.7]}\right)\right), \\
& \left.\left(k_{3},\left(\frac{\left(u_{1}, 6\right)}{[0.1,0.2],[0.4,0.5]}, \frac{\left(u_{2}, 3\right)}{[0.8,0.9],[0.7,0.8]}, \frac{\left(u_{3}, 5\right)}{[0.8,0.9],[0.3,0.6]}\right)\right)\right\} .
\end{aligned}
$$




$$
\begin{aligned}
\left(Y_{y}, L, 6\right)=\{ & \left(k_{2},\left(\frac{\left(u_{1}, 2\right)}{[0.2,0.6],[0.4,0.5]}, \frac{\left(u_{2}, 1\right)}{[0.2,0.5],[0.1,0.7]}, \frac{\left(u_{3}, 4\right)}{[0.2,0.7],[0.3,0.7]}\right)\right), \\
& \left.\left(k_{4},\left(\frac{\left(u_{1}, 0\right)}{[0.6,0.9],[0.5,0.8]}, \frac{\left(u_{2}, 5\right)}{[0.5,0.7],[0.4,0.8]}, \frac{\left(u_{3}, 3\right)}{[0.3,0.9],[0.1,0.9]}\right)\right)\right\} .
\end{aligned}
$$

Then their restricted intersection is:

$$
\left(D_{d}, T, 6\right)=\left\{\left(k_{2},\left(\frac{\left(u_{1}, 2\right)}{[0.2,0.6],[0.4,0.5]}, \frac{\left(u_{2}, 0\right)}{[0.1,0.5],[0.1,0.7]}, \frac{\left(u_{3}, 4\right)}{[0.2,0.7],[0.3,0.7]}\right)\right)\right\} .
$$

their restricted union is:

$$
\left(E_{e}, T, 7\right)=\left\{\left(k_{2},\left(\frac{\left(u_{1}, 4\right)}{[0.6,0.7],[0.5,0.7]}, \frac{\left(u_{2}, 1\right)}{[0.2,0.8],[0.7,0.9]}, \frac{\left(u_{3}, 6\right)}{[0.6,0.8],[0.4,0.7]}\right)\right)\right\} .
$$

their extended intersection is

$$
\begin{aligned}
\left(F_{f}, S, 7\right)=\{ & \left(k_{1},\left(\frac{\left(u_{1}, 1\right)}{[0.3,0.8],[0.3,0.7]}, \frac{\left(u_{2}, 5\right)}{[0.3,0.4],[0.1,0.5]}, \frac{\left(u_{3}, 2\right)}{[0.4,0.6],[0.3,0.5]}\right)\right), \\
& \left(k_{2},\left(\frac{\left(u_{1}, 2\right)}{[0.2,0.6],[0.4,0.5]}, \frac{\left(u_{2}, 0\right)}{[0.1,0.5],[0.1,0.7]}, \frac{\left(u_{3}, 4\right)}{[0.2,0.7],[0.3,0.7]}\right)\right), \\
& \left(k_{3},\left(\frac{\left(u_{1}, 6\right)}{[0.1,0.2],[0.4,0.5]}, \frac{\left(u_{2}, 3\right)}{[0.8,0.9],[0.7,0.8]}, \frac{\left(u_{3}, 5\right)}{[0.8,0.9],[0.3,0.6]}\right)\right), \\
& \left.\left(k_{4},\left(\frac{\left(u_{1}, 0\right)}{[0.6,0.9],[0.5,0.8]}, \frac{\left(u_{2}, 5\right)}{[0.5,0.7],[0.4,0.8]}, \frac{\left(u_{3}, 3\right)}{[0.3,0.9],[0.1,0.9]}\right)\right)\right\} .
\end{aligned}
$$

their extended union is:

$$
\begin{aligned}
\left(H_{h}, S, 7\right)=\{ & \left(k_{1},\left(\frac{\left(u_{1}, 1\right)}{[0.3,0.8],[0.3,0.7]}, \frac{\left(u_{2}, 5\right)}{[0.3,0.4],[0.1,0.5]}, \frac{\left(u_{3}, 2\right)}{[0.4,0.6],[0.3,0.5]}\right)\right), \\
& \left(k_{2},\left(\frac{\left(u_{1}, 4\right)}{[0.6,0.7],[0.5,0.7]}, \frac{\left(u_{2}, 1\right)}{[0.2,0.8],[0.7,0.9]}, \frac{\left(u_{3}, 6\right)}{[0.6,0.8],[0.4,0.7]}\right)\right), \\
& \left(k_{3},\left(\frac{\left(u_{1}, 6\right)}{[0.1,0.2],[0.4,0.5]}, \frac{\left(u_{2}, 3\right)}{[0.8,0.9],[0.7,0.8]}, \frac{\left(u_{3}, 5\right)}{[0.8,0.9],[0.3,0.6]}\right)\right), \\
& \left.\left(k_{4},\left(\frac{\left(u_{1}, 0\right)}{[0.6,0.9],[0.5,0.8]}, \frac{\left(u_{2}, 5\right)}{[0.5,0.7],[0.4,0.8]}, \frac{\left(u_{3}, 3\right)}{[0.3,0.9],[0.1,0.9]}\right)\right)\right\} .
\end{aligned}
$$

Definition 22. Let $\left(B_{b}, K, N\right)$ be a PBIVNSS on a non-empty universe $U$. Then a weak possibility belief interval-valued complement is denoted by $\left(B_{b c}^{c}, K, N\right)$ where $B_{b c}^{c}\left(k_{j}\right) \cap B_{b}\left(k_{j}\right)=\Phi ; \forall k_{j} \in K$ and $B_{b^{c}}^{c}\left(k_{j}\right)$ is defined as:

$$
\begin{gathered}
B_{b^{c}}^{c}\left(k_{j}\right)=\left\{\frac{\left(u_{i}, r_{i j}\right)}{B I_{B^{c}\left(k_{j}\right)}\left(u_{i}, r_{i j}\right), B I_{b^{c}\left(k_{j}\right)}\left(u_{i}, r_{i j}\right)} \mid\left(u_{i}, r_{i j}\right) \in U \times R\right\}, \\
\text { where, } \quad B I_{B^{c}\left(k_{j}\right)}\left(u_{i}, r_{i j}\right)=\left[1-P l_{B\left(k_{j}\right)}\left(u_{i}, r_{i j}\right), 1-B e l_{B\left(k_{j}\right)}\left(u_{i}, r_{i j}\right)\right], \\
B I_{b^{c}\left(k_{j}\right)}\left(u_{i}, r_{i j}\right)=\left[1-P l_{b\left(k_{j}\right)}\left(u_{i}, r_{i j}\right), 1-B e l_{b\left(k_{j}\right)}\left(u_{i}, r_{i j}\right)\right] .
\end{gathered}
$$

Definition 23. For any PBIVNSS $\left(B_{b}, K, N\right)$ on $U$. The bottom weak possibility belief intervalvalued complement $\left(B_{b}^{\prec}, K, N\right)$ of $\left(B_{b}, K, N\right)$ is defined as: $\left(B_{b}^{\prec}, K, N\right)=B_{b}\left(k_{j}\right)=$

$$
\left\{\begin{array}{c}
\frac{\left(u_{i}, 0\right)}{B I_{B^{c}\left(k_{j}\right)}\left(u_{i}, r_{i j}\right), B I_{b^{c}\left(k_{j}\right)}\left(u_{i}, r_{i j}\right)}, \text { if } r_{i j}>0, \\
\frac{\left(u_{i}, N-1\right)}{B I_{B^{c}\left(k_{j}\right)}\left(u_{i}, r_{i j}\right), B I_{b^{c}\left(k_{j}\right)}\left(u_{i}, r_{i j}\right)}, \text { if } r_{i j}=0 .
\end{array}\right.
$$


Definition 24. For any PBIVNSS $\left(B_{b}, K, N\right)$ on $U$. The top weak possibility belief intervalvalued complement $\left(B_{b}^{\succ}, K, N\right)$ of $\left(B_{b}, K, N\right)$ is defined as:

$$
\left(B_{b}^{\succ}, K, N\right)=B_{b}\left(k_{j}\right)=\left\{\begin{array}{l}
\frac{\left(u_{i}, N-1\right)}{B I_{B^{c}\left(k_{j}\right)}\left(u_{i}, r_{i j}\right), B I_{b^{c}\left(k_{j}\right)}\left(u_{i}, r_{i j}\right)}, \text { if } r_{i j}<N-1, \\
\frac{\left(u_{i}, 0\right)}{B I_{B^{c}\left(k_{j}\right)}\left(u_{i}, r_{i j}\right), B I_{b^{c}\left(k_{j}\right)}\left(u_{i}, r_{i j}\right)}, \text { if } r_{i j}=N-1 .
\end{array}\right.
$$

Example 9. Consider $\left(B_{b}, K, 7\right)$ as described in Example 7 , then its weak possibility belief intervalvalued complement is:

$$
\begin{aligned}
\left(B_{b c}^{c}, K, 7\right)=\{ & \left(k_{1},\left(\frac{\left(u_{1}, 1\right)}{[0.2,0.7],[0.3,0.7]}, \frac{\left(u_{2}, 5\right)}{[0.6,0.7],[0.5,0.9]}, \frac{\left(u_{3}, 2\right)}{[0.4,0.6],[0.5,0.7]}\right)\right) \\
& \left(k_{2},\left(\frac{\left(u_{1}, 4\right)}{[0.3,0.4],[0.3,0.5]}, \frac{\left(u_{2}, 0\right)}{[0.2,0.9],[0.1,0.3]}, \frac{\left(u_{3}, 6\right)}{[0.2,0.4],[0.3,0.6]}\right)\right) \\
& \left.\left(k_{3},\left(\frac{\left(u_{1}, 6\right)}{[0.8,0.9],[0.5,0.6]}, \frac{\left(u_{2}, 3\right)}{[0.1,0.2],[0.2,0.3]}, \frac{\left(u_{3}, 5\right)}{[0.1,0.2],[0.4,0.7]}\right)\right)\right\}
\end{aligned}
$$

its bottom weak possibility belief interval-valued complement is:

$$
\begin{aligned}
\left(B_{b}^{\prec}, K, 7\right)=\{ & \left(k_{1},\left(\frac{\left(u_{1}, 0\right)}{[0.2,0.7],[0.3,0.7]}, \frac{\left(u_{2}, 0\right)}{[0.6,0.7],[0.5,0.9]}, \frac{\left(u_{3}, 0\right)}{[0.4,0.6],[0.5,0.7]}\right)\right), \\
& \left(k_{2},\left(\frac{\left(u_{1}, 0\right)}{[0.3,0.4],[0.3,0.5]}, \frac{\left(u_{2}, 6\right)}{[0.2,0.9],[0.1,0.3]}, \frac{\left(u_{3}, 0\right)}{[0.2,0.4],[0.3,0.6]}\right)\right), \\
& \left.\left(k_{3},\left(\frac{\left(u_{1}, 0\right)}{[0.8,0.9],[0.5,0.6]}, \frac{\left(u_{2}, 0\right)}{[0.1,0.2],[0.2,0.3]}, \frac{\left(u_{3}, 0\right)}{[0.1,0.2],[0.4,0.7]}\right)\right)\right\} .
\end{aligned}
$$

its top weak possibility belief interval-valued complement is:

$$
\begin{aligned}
\left(B_{b}^{\prec}, K, 7\right)=\{ & \left(k_{1},\left(\frac{\left(u_{1}, 6\right)}{[0.2,0.7],[0.3,0.7]}, \frac{\left(u_{2}, 6\right)}{[0.6,0.7],[0.5,0.9]}, \frac{\left(u_{3}, 6\right)}{[0.4,0.6],[0.5,0.7]}\right)\right), \\
& \left(k_{2},\left(\frac{\left(u_{1}, 6\right)}{[0.3,0.4],[0.3,0.5]}, \frac{\left(u_{2}, 6\right)}{[0.2,0.9],[0.1,0.3]}, \frac{\left(u_{3}, 0\right)}{[0.2,0.4],[0.3,0.6]}\right)\right), \\
& \left.\left(k_{3},\left(\frac{\left(u_{1}, 0\right)}{[0.8,0.9],[0.5,0.6]}, \frac{\left(u_{2}, 6\right)}{[0.1,0.2],[0.2,0.3]}, \frac{\left(u_{3}, 6 s\right)}{[0.1,0.2],[0.4,0.7]}\right)\right)\right\} .
\end{aligned}
$$

Definition 25. Soft max-AND operation of two PBIVNSS $\left(B_{b}, K, M\right)$ and $\left(Y_{y}, L, N\right)$ (where $B_{b}: K \rightarrow B I^{U \times R} \times B I^{U \times R}$ and $\left.Y_{y}: L \rightarrow B I^{U \times R} \times B I^{U \times R}\right)$ defined as:

$$
\left(B_{b}, K, M\right) \widehat{\wedge}\left(Y_{y}, L, N\right)=\left(G_{g}, K \times L, P\right),
$$

where $G_{g}: K \times L \rightarrow B I^{U \times R} \times B I^{U \times R} ; \forall\left(k_{s^{\prime}}, l_{t^{\prime}}\right) \in(K \times L), s^{\prime}, t^{\prime} \in \Lambda$, and $P=\max (M, N)$,

$$
\begin{gathered}
G_{g}\left(k_{s^{\prime}}, l_{t^{\prime}}\right) \in \frac{\left(u_{i}, r_{i\left(s^{\prime}, t^{\prime}\right)}\right)}{B I_{G\left(k_{s^{\prime}}, l_{t^{\prime}}\right)}\left(u_{i}, r_{i\left(s^{\prime}, t^{\prime}\right)}\right), B I_{g\left(k_{s^{\prime}}, l_{t^{\prime}}\right)}\left(u_{i}, r_{i\left(s^{\prime}, t^{\prime}\right)}\right)} \Leftrightarrow r_{i\left(s^{\prime}, t^{\prime}\right)}=\max \left(r_{i\left(s^{\prime}, t^{\prime}\right)}^{1}, r_{i\left(s^{\prime}, t^{\prime}\right)}^{2}\right), \\
B I_{G\left(k_{s^{\prime}}, l_{t^{\prime}}\right)}\left(u_{i}, r_{i\left(s^{\prime}, t^{\prime}\right)}\right)=\left[\operatorname{Bel}\left(k_{s^{\prime}}, l_{t^{\prime}}\right)\left(u_{i}, r_{i\left(s^{\prime}, t^{\prime}\right)}\right), P l_{G\left(k_{s^{\prime}}, l_{t^{\prime}}\right)}\left(u_{i}, r_{i\left(s^{\prime}, t^{\prime}\right)}\right)\right], \\
B I_{g\left(k_{s^{\prime}}, l_{t^{\prime}}\right)}\left(u_{i}, r_{i\left(s^{\prime}, t^{\prime}\right)}\right)=\left[B e l_{g\left(k_{s^{\prime}}, l_{t^{\prime}}\right)}\left(u_{i}, r_{i\left(s^{\prime}, t^{\prime}\right)}\right), P l_{g\left(k_{s^{\prime}}, l_{t^{\prime}}\right)}\left(u_{i}, r_{i\left(s^{\prime}, t^{\prime}\right)}\right)\right] .
\end{gathered}
$$


where,

$$
\begin{aligned}
{\left[\operatorname{BelG}\left(k_{s^{\prime}}, l_{t^{\prime}}\right)\left(u_{i}, r_{i\left(s^{\prime}, t^{\prime}\right)}\right),\right.} & \left.P l_{G\left(k_{s^{\prime}}, l_{t^{\prime}}\right)}\left(u_{i}, r_{i\left(s^{\prime}, t^{\prime}\right)}\right)\right]= \\
& {\left[\frac{2}{3}\left(\operatorname{Bel}_{B\left(k_{s^{\prime}}\right)}\left(u_{i}, r_{i\left(s^{\prime}, t^{\prime}\right)}\right)+\operatorname{Bel}_{Y\left(l_{t^{\prime}}\right)}\left(u_{i}, r_{i\left(s^{\prime}, t^{\prime}\right)}\right)\right)-\right.} \\
& \frac{1}{3} \max \left\{\operatorname{Bel}_{B\left(k_{s^{\prime}}\right)}\left(u_{i}, r_{i\left(s^{\prime}, t^{\prime}\right)}, \operatorname{Bel} l_{Y\left(l_{t^{\prime}}\right)}\left(u_{i}, r_{i\left(s^{\prime}, t^{\prime}\right)}\right)\right\},\right. \\
& \frac{2}{3}\left(P l_{B\left(k_{s^{\prime}}\right)}\left(u_{i}, r_{i\left(s^{\prime}, t^{\prime}\right)}\right)+P l_{Y\left(l_{t^{\prime}}\right)}\left(u_{i}, r_{i\left(s^{\prime}, t^{\prime}\right)}\right)\right)- \\
& \left.\frac{1}{3} \max \left\{P l_{B\left(k_{s^{\prime}}\right)}\left(u_{i}, r_{i\left(s^{\prime}, t^{\prime}\right)}\right), P l_{Y\left(l_{t^{\prime}}\right)}\left(u_{i}, r_{i\left(s^{\prime}, t^{\prime}\right)}\right)\right\}\right]
\end{aligned}
$$

and

$$
\begin{aligned}
{\left[\operatorname{Bel}_{g\left(k_{s^{\prime}}, l_{t^{\prime}}\right)}\left(u_{i}, r_{i\left(s^{\prime}, t^{\prime}\right)}\right), P l_{g\left(k_{s^{\prime}}, l_{t^{\prime}}\right)}\left(u_{i}, r_{i\left(s^{\prime}, t^{\prime}\right)}\right)\right]=} & \\
& {\left[\frac{2}{3}\left(\operatorname{Bel}_{b\left(k_{s^{\prime}}\right)}\left(u_{i}, r_{i\left(s^{\prime}, t^{\prime}\right)}\right)+\operatorname{Bel}_{y\left(l_{t^{\prime}}\right)}\left(u_{i}, r_{i\left(s^{\prime}, t^{\prime}\right)}\right)\right)-\right.} \\
& \frac{1}{3} \max \left\{B e l_{b\left(k_{s^{\prime}}\right)}\left(u_{i}, r_{i\left(s^{\prime}, t^{\prime}\right)}\right), B e l_{y\left(l_{t^{\prime}}\right)}\left(u_{i}, r_{i\left(s^{\prime}, t^{\prime}\right)}\right)\right\}, \\
& \frac{2}{3}\left(P l_{b\left(k_{s^{\prime}}\right)}\left(u_{i}, r_{i\left(s^{\prime}, t^{\prime}\right)}\right)+P l_{y\left(l_{t^{\prime}}\right)}\left(u_{i}, r_{i\left(s^{\prime}, t^{\prime}\right)}\right)\right)- \\
& \left.\frac{1}{3} \max \left\{P l_{b\left(k_{s^{\prime}}\right)}\left(u_{i}, r_{i\left(s^{\prime}, t^{\prime}\right)}\right), P l_{y\left(l_{t^{\prime}}\right)}\left(u_{i}, r_{i\left(s^{\prime}, t^{\prime}\right)}\right)\right\}\right]
\end{aligned}
$$

with $\left(u_{i}, r_{i\left(s^{\prime}, t^{\prime}\right)}^{1}\right) \in B_{b}(K)$ and $\left(u_{i}, r_{i\left(s^{\prime}, t^{\prime}\right)}^{2}\right) \in Y_{y}(L)$.

Definition 26. Soft min-OR operation of two PBIVNSS $\left(B_{b}, K, M\right)$ and $\left(Y_{y}, L, N\right)$ (where $B_{b}$ : $K \rightarrow B I^{U \times R} \times B I^{U \times R}$ and $\left.Y_{y}: L \rightarrow B I^{U \times R} \times B I^{U \times R}\right)$ defined as:

$$
\left(B_{b}, K, M\right) \widehat{\nabla}\left(Y_{y}, L, N\right)=\left(Q_{q}, K \times L, O\right),
$$

where $Q_{q}: K \times L \rightarrow B I^{U \times R} \times B I^{U \times R} ; \forall\left(k_{s^{\prime}}, l_{t^{\prime}}\right) \in(K \times L), s^{\prime}, t^{\prime} \in \Lambda$, and $O=\min (M, N)$,

$$
\begin{gathered}
Q_{q}\left(k_{s^{\prime}}, l_{t^{\prime}}\right) \in \frac{\left(u_{i}, r_{i\left(s^{\prime}, t^{\prime}\right)}\right)}{B I_{Q\left(k_{s^{\prime}}, l_{t^{\prime}}\right)}\left(u_{i}, r_{i\left(s^{\prime}, t^{\prime}\right)}\right), B I_{q\left(k_{s^{\prime}}, l_{t^{\prime}}\right)}\left(u_{i}, r_{i\left(s^{\prime}, t^{\prime}\right)}\right)} \Leftrightarrow r_{i\left(s^{\prime}, t^{\prime}\right)}=\min \left(r_{i\left(s^{\prime}, t^{\prime}\right)}^{1}, r_{i\left(s^{\prime}, t^{\prime}\right)}^{2}\right), \\
B I_{Q\left(k_{s^{\prime}}, l_{t^{\prime}}\right)}\left(u_{i}, r_{i\left(s^{\prime}, t^{\prime}\right)}\right)=\left[B e l_{Q\left(k_{s^{\prime}}, l_{t^{\prime}}\right)}\left(u_{i}, r_{i\left(s^{\prime}, t^{\prime}\right)}\right), P l_{Q\left(k_{s^{\prime}}, l_{t^{\prime}}\right)}\left(u_{i}, r_{i\left(s^{\prime}, t^{\prime}\right)}\right)\right], \\
B I_{q\left(k_{s^{\prime}}, l_{t^{\prime}}\right)}\left(u_{i}, r_{i\left(s^{\prime}, t^{\prime}\right)}\right)=\left[B e l_{q\left(k_{s^{\prime}}, l_{t^{\prime}}\right)}\left(u_{i}, r_{i\left(s^{\prime}, t^{\prime}\right)}\right), P l_{q\left(k_{s^{\prime}}, l_{t^{\prime}}\right)}\left(u_{i}, r_{i\left(s^{\prime}, t^{\prime}\right)}\right)\right] .
\end{gathered}
$$

where,

$$
\begin{aligned}
{\left[\operatorname{Bel}_{Q\left(k_{s^{\prime}}, l_{t^{\prime}}\right)}\left(u_{i}, r_{i\left(s^{\prime}, t^{\prime}\right)}\right),\right.} & \left.P l_{Q\left(k_{s^{\prime}}, l_{t^{\prime}}\right)}\left(u_{i}, r_{i\left(s^{\prime}, t^{\prime}\right)}\right)\right]= \\
& {\left[\frac{2}{3}\left(\operatorname{Bel}_{B\left(k_{s^{\prime}}\right)}\left(u_{i}, r_{i\left(s^{\prime}, t^{\prime}\right)}\right)+\operatorname{Bel}_{Y\left(l_{t^{\prime}}\right)}\left(u_{i}, r_{i\left(s^{\prime}, t^{\prime}\right)}\right)\right)-\right.} \\
& \frac{1}{3} \min \left\{B e l_{B\left(k_{s^{\prime}}\right)}\left(u_{i}, r_{i\left(s^{\prime}, t^{\prime}\right)}\right), \operatorname{Bel}_{Y\left(l_{t^{\prime}}\right)}\left(u_{i}, r_{i\left(s^{\prime}, t^{\prime}\right)}\right)\right\}, \\
& \frac{2}{3}\left(P l_{B\left(k_{s^{\prime}}\right)}\left(u_{i}, r_{i\left(s^{\prime}, t^{\prime}\right)}\right)+P l_{Y\left(l_{t^{\prime}}\right)}\left(u_{i}, r_{i\left(s^{\prime}, t^{\prime}\right)}\right)\right)- \\
& \left.\frac{1}{3} \min \left\{P l_{B\left(k_{s^{\prime}}\right)}\left(u_{i}, r_{i\left(s^{\prime}, t^{\prime}\right)}\right), P l_{Y\left(l_{t^{\prime}}\right)}\left(u_{i}, r_{i\left(s^{\prime}, t^{\prime}\right)}\right)\right\}\right]
\end{aligned}
$$


and

$$
\begin{aligned}
{\left[\operatorname{Bel}_{q\left(k_{s^{\prime}}, l_{t^{\prime}}\right)}\left(u_{i}, r_{i\left(s^{\prime}, t^{\prime}\right)}\right), P l_{q\left(k_{s^{\prime}}, l_{t^{\prime}}\right)}\left(u_{i}, r_{i\left(s^{\prime}, t^{\prime}\right)}\right)\right]=} & \\
& {\left[\frac{2}{3}\left(\operatorname{Bel}_{b\left(k_{s^{\prime}}\right)}\left(u_{i}, r_{i\left(s^{\prime}, t^{\prime}\right)}\right)+\operatorname{Bel}_{y\left(l_{t^{\prime}}\right)}\left(u_{i}, r_{i\left(s^{\prime}, t^{\prime}\right)}\right)\right)-\right.} \\
& \frac{1}{3} \min \left\{B e l_{b\left(k_{s^{\prime}}\right)}\left(u_{i}, r_{i\left(s^{\prime}, t^{\prime}\right)}\right), B e l_{y\left(l_{t^{\prime}}\right)}\left(u_{i}, r_{i\left(s^{\prime}, t^{\prime}\right)}\right)\right\}, \\
& \frac{2}{3}\left(P l_{b\left(k_{s^{\prime}}\right)}\left(u_{i}, r_{i\left(s^{\prime}, t^{\prime}\right)}\right)+P l_{y\left(l_{t^{\prime}}\right)}\left(u_{i}, r_{i\left(s^{\prime}, t^{\prime}\right)}\right)\right)- \\
& \left.\frac{1}{3} \min \left\{P l_{b\left(k_{s^{\prime}}\right)}\left(u_{i}, r_{i\left(s^{\prime}, t^{\prime}\right)}\right), P l_{y\left(l_{t^{\prime}}\right)}\left(u_{i}, r_{i\left(s^{\prime}, t^{\prime}\right)}\right)\right\}\right]
\end{aligned}
$$

with $\left(u_{i}, r_{i\left(s^{\prime}, t^{\prime}\right)}^{1}\right) \in B_{b}(K)$ and $\left(u_{i}, r_{i\left(s^{\prime}, t^{\prime}\right)}^{2}\right) \in Y_{y}(L)$.

Example 10. Consider $\left(B_{b}, K, 7\right)$ and $\left(Y_{y}, L, 6\right)$ as described in Example 8, then their soft maxAND is:

$$
\begin{aligned}
\left(G_{g}, K \times L, 7\right)=\{ & \left(\left(k_{1}, k_{2}\right),\left(\frac{\left(u_{1}, 2\right)}{[0.23,0.66],[0.33,0.56]}, \frac{\left(u_{2}, 5\right)}{[0.23,0.43],[0.10,0.56]}, \frac{\left(u_{3}, 4\right)}{[0.26,0.63],[0.30,0.56]}\right)\right), \\
& \left(\left(k_{1}, k_{4}\right),\left(\frac{\left(u_{1}, 1\right)}{[0.40,0.83],[0.36,0.73]}, \frac{\left(u_{2}, 5\right)}{[0.36,0.50],[0.20,0.60]}, \frac{\left(u_{3}, 3\right)}{[0.33,0.70],[0.16,0.63]}\right)\right), \\
& \left(\left(k_{2}, k_{2}\right),\left(\frac{\left(u_{1}, 4\right)}{[0.33,0.63],[0.43,0.56]}, \frac{\left(u_{2}, 1\right)}{[0.13,0.60],[0.30,0.76]}, \frac{\left(u_{3}, 6\right)}{[0.33,0.73],[0.33,0.70]}\right)\right), \\
& \left(\left(k_{2}, k_{4}\right),\left(\frac{\left(u_{1}, 4\right)}{[0.60,0.76],[0.50,0.73]}, \frac{\left(u_{2}, 5\right)}{[0.23,0.73],[0.50,0.83]}, \frac{\left(u_{3}, 6\right)}{[0.40,0.83],[0.20,0.76]}\right)\right), \\
& \left(\left(k_{3}, k_{2}\right),\left(\frac{\left(u_{1}, 6\right)}{[0.13,0.33],[0.40,0.50]}, \frac{\left(u_{2}, 3\right)}{[0.40,0.63],[0.30,0.73]}, \frac{\left(u_{3}, 5\right)}{[0.40,0.76],[0.30,0.63]}\right)\right), \\
& \left.\left(\left(k_{3}, k_{4}\right),\left(\frac{\left(u_{1}, 6\right)}{[0.26,0.43],[0.43,0.60]}, \frac{\left(u_{2}, 5\right)}{[0.60,0.76],[0.50,0.80]}, \frac{\left(u_{3}, 5\right)}{[0.46,0.90],[0.16,0.70]}\right)\right)\right\} .
\end{aligned}
$$

their soft min-OR is:

$$
\begin{aligned}
\left(Q_{q}, K \times L, 6\right)=\{ & \left(\left(k_{1}, k_{2}\right),\left(\frac{\left(u_{1}, 1\right)}{[0.26,0.73],[0.36,0.63]}, \frac{\left(u_{2}, 1\right)}{[0.26,0.46],[0.10,0.63]}, \frac{\left(u_{3}, 2\right)}{[0.33,0.66],[0.30,0.63]}\right)\right), \\
& \left(\left(k_{1}, k_{4}\right),\left(\frac{\left(u_{1}, 0\right)}{[0.50,0.86],[0.43,0.76]}, \frac{\left(u_{2}, 5\right)}{[0.43,0.60],[0.30,0.70]}, \frac{\left(u_{3}, 2\right)}{[0.36,0.80],[0.23,0.76]}\right)\right), \\
& \left(\left(k_{2}, k_{2}\right),\left(\frac{\left(u_{1}, 2\right)}{[0.46,0.66],[0.46,0.63]}, \frac{\left(u_{2}, 0\right)}{[0.16,0.70],[0.50,0.83]}, \frac{\left(u_{3}, 4\right)}{[0.46,0.76],[0.36,0.70]}\right)\right), \\
& \left(\left(k_{2}, k_{4}\right),\left(\frac{\left(u_{1}, 0\right)}{[0.60,0.83],[0.50,0.76]}, \frac{\left(u_{2}, 0\right)}{[0.36,0.76],[0.60,0.86]}, \frac{\left(u_{3}, 3\right)}{[0.50,0.86],[0.30,0.83]}\right)\right), \\
& \left(\left(k_{3}, k_{2}\right),\left(\frac{\left(u_{1}, 2\right)}{[0.16,0.46],[0.40,0.50]}, \frac{\left(u_{2}, 1\right)}{[0.60,0.76],[0.50,0.76]}, \frac{\left(u_{3}, 4\right)}{[0.60,0.83],[0.30,0.66]}\right)\right), \\
& \left.\left(\left(k_{3}, k_{4}\right),\left(\frac{\left(u_{1}, 0\right)}{[0.43,0.66],[0.46,0.70]}, \frac{\left(u_{2}, 3\right)}{[0.70,0.83],[0.60,0.80]}, \frac{\left(u_{3}, 3\right)}{[0.63,0.90],[0.23,0.80]}\right)\right)\right\} .
\end{aligned}
$$

Proposition 3. Given that $\left(B_{b}, K, M\right),\left(Y_{y}, L, N\right)$, and $\left(D_{d}, T, O\right)$ are any three BIVNSS on $U$, then the commutative and associative properties hold:

(1) $\left(B_{b}, K, M\right) \sqcap_{E}\left(B_{b}, K, M\right)=\left(B_{b}, K, M\right)$,

(2) $\left(B_{b}, K, M\right) \sqcap_{R}\left(B_{b}, K, M\right)=\left(B_{b}, K, M\right)$,

(3) $\left(B_{b}, K, M\right) \sqcup_{E}\left(B_{b}, K, M\right)=\left(B_{b}, K, M\right)$,

(4) $\left(B_{b}, K, M\right) \sqcup_{R}\left(B_{b}, K, M\right)=\left(B_{b}, K, M\right)$,

(5) $\left(B_{b}, K, M\right) \sqcap_{E}\left(Y_{y}, L, N\right)=\left(Y_{y}, L, N\right) \sqcap_{E}\left(B_{b}, K, M\right)$,

(6) $\left(B_{b}, K, M\right) \sqcap_{R}\left(Y_{y}, L, N\right)=\left(Y_{y}, L, N\right) \sqcap_{R}\left(B_{b}, K, M\right)$,

(7) $\left(B_{b}, K, M\right) \sqcup_{E}\left(Y_{y}, L, N\right)=\left(Y_{y}, L, N\right) \sqcup_{E}\left(B_{b}, K, M\right)$,

(8) $\left(B_{b}, K, M\right) \sqcup_{R}\left(Y_{y}, L, N\right)=\left(Y_{y}, L, N\right) \sqcup_{R}\left(B_{b}, K, M\right)$,

(9) $\left(B_{b}, K, M\right) \sqcap_{E}\left(\left(Y_{y}, L, N\right) \sqcap_{E}\left(D_{d}, T, O\right)\right)=\left(\left(B_{b}, K, M\right) \sqcap_{E}\left(Y_{y}, L, N\right)\right) \sqcap_{E}\left(D_{d}, T, O\right)$, 
(10) $\left(B_{b}, K, M\right) \sqcap_{R}\left(\left(Y_{y}, L, N\right) \sqcap_{R}\left(D_{d}, T, O\right)\right)=\left(\left(B_{b}, K, M\right) \sqcap_{R}\left(Y_{y}, L, N\right)\right) \sqcap_{R}\left(D_{d}, T, O\right)$,

(11) $\left(B_{b}, K, M\right) \sqcup_{E}\left(\left(Y_{y}, L, N\right) \sqcup_{E}\left(D_{d}, T, O\right)\right)=\left(\left(B_{b}, K, M\right) \sqcup_{E}\left(Y_{y}, L, N\right)\right) \sqcup_{E}\left(D_{d}, T, O\right)$,

(12) $\left(B_{b}, K, M\right) \sqcup_{R}\left(\left(Y_{y}, L, N\right) \sqcup_{R}\left(D_{d}, T, O\right)\right)=\left(\left(B_{b}, K, M\right) \sqcup_{R}\left(Y_{y}, L, N\right)\right) \sqcup_{R}\left(D_{d}, T, O\right)$.

Proof. (1)-(8) follows from definition. For (9), let $\left(Y_{y}, L, N\right)$ and $\left(D_{d}, T, O\right)$ (where $Y_{y}$ : $L \rightarrow B I^{U \times R} \times B I^{U \times R}$ and $\left.D_{d}: T \rightarrow B I^{U \times R} \times B I^{U \times R}\right)$ be two PBIVNSS by the definition of extended intersection we have $\left(S_{s}, V, Z\right.$ ) (where $S_{s}: V \rightarrow B I^{U \times R} \times B I^{U \times R}$ ) such that $\left(S_{s}, V, Z\right)=\left(Y_{y}, L, N\right) \cap_{E}\left(D_{d}, T, O\right)$, where $S_{s}=Y_{y} \sqcap_{E} D_{d}, V=L \cup T, Z=$ $\max (N, O) ; \forall v_{j} \in V$ with $v_{j}^{1} \in L$ and $v_{j}^{2} \in T$,

$$
S_{s}\left(v_{j}\right)= \begin{cases}\Upsilon_{y}\left(v_{j}^{1}\right) & , \text { if } v_{j} \in L-T, \\ D_{d}\left(v_{j}^{2}\right) & , \text { if } v_{j} \in T-L, \\ Y_{y}\left(v_{j}^{1}\right) \sqcap_{R} D_{d}\left(v_{j}^{2}\right) & , \text { if } v_{j} \in L \cap T .\end{cases}
$$

As, $\left(B_{b}, K, M\right) \sqcap_{E}\left(\left(Y_{y}, L, N\right) \sqcap_{E}\left(D_{d}, T, O\right)\right)=\left(B_{b}, K, M\right) \sqcap_{E}\left(S_{s}, V, Z\right)$. Suppose $\left(B_{b}, K, M\right)$ $\Pi_{E}\left(S_{S}, V, Z\right)=\left(G_{g}, W, X\right)$ such that $G_{g}: W \rightarrow B I^{U \times R} \times B I^{U \times R}$, where $G_{g}=B_{b} \sqcap_{E}$ $S_{s}, W=K \cup V=K \cup L \cup T, X=\max (M, Z) ; \forall w_{j} \in W$ with $w_{j}^{1} \in K, w_{j}^{2} \in L$ and

$$
w_{j}^{3} \in T, G_{g}\left(w_{j}\right)= \begin{cases}B_{b}\left(w_{j}^{1}\right) & , \text { if } w_{j} \in K-L-T, \\ Y_{y}\left(w_{j}^{2}\right) & , \text { if } w_{j} \in L-K-T, \\ D_{d}\left(w_{j}^{3}\right) & , \text { if } w_{j} \in T-K-L, \\ Y_{y}\left(w_{j}^{2}\right) \sqcap_{R} D_{d}\left(w_{j}^{3}\right) & , \text { if } w_{j} \in L \cap T-K, \\ B_{b}\left(w_{j}^{1}\right) \sqcap_{R} Y_{y}\left(w_{j}^{2}\right) & , \text { if } w_{j} \in K \cap L-T, \\ B_{b}\left(w_{j}^{1}\right) \sqcap_{R} D_{d}\left(w_{j}^{3}\right) & , \text { if } w_{j} \in K \cap T-L, \\ B_{b}\left(w_{j}^{1}\right) \sqcap_{R} Y_{y}\left(w_{j}^{2}\right) \sqcap_{R} D_{d}\left(w_{j}^{3}\right) & , \text { if } w_{j} \in K \cap L \cap T .\end{cases}
$$

Again, let $\left(B_{b}, K, M\right)$ and $\left(Y_{y}, L, N\right)$ (where $B_{b}: K \rightarrow B I^{U \times R} \times B I^{U \times R}$ and $Y_{y}: L \rightarrow$ $\left.B I^{U \times R} \times B I^{U \times R}\right)$ be two PBIVNSS by the definition of extended intersection we have $\left(F_{f}, S, P\right)$ (where $\left.F_{f}: S \rightarrow B I^{U \times R} \times B I^{U \times R}\right)$ such that $\left(F_{f}, S, P\right)=\left(B_{b}, K, M\right) \cap_{E}\left(Y_{y}, L, N\right)$, where $F_{f}=B_{b} \sqcap_{E} Y_{y}, S=K \cup L, P=\max (M, N) ; \forall s_{j} \in S$ with $s_{j}^{1} \in K$ and $s_{j}^{2} \in L$,

$$
F_{f}\left(s_{j}\right)= \begin{cases}B_{b}\left(s_{j}^{1}\right) & , \text { if } s_{j} \in K-L, \\ Y_{y}\left(s_{j}^{2}\right) & , \text { if } s_{j} \in L-K, \\ B_{b}\left(s_{j}^{1}\right) \sqcap_{R} Y_{y}\left(s_{j}^{2}\right) & , \text { if } s_{j} \in K \cap L .\end{cases}
$$

As, $\left(\left(B_{b}, K, M\right) \sqcap_{E}\left(Y_{y}, L, N\right)\right) \sqcap_{E}\left(D_{d}, T, O\right)=\left(F_{f}, S, P\right) \sqcap_{E}\left(D_{d}, T, O\right)$. Suppose $\left(F_{f}, S, P\right)$ $\Pi_{E}\left(D_{d}, T, O\right)=\left(I_{i}, W, X\right)$ such that $I_{i}: W \rightarrow B I^{U \times R} \times B I^{U \times R}$, where $I_{i}=F_{f} \sqcap_{E} D_{d}, W=$ $S \cup T=K \cup L \cup T, X=\max (P, O) ; \forall w_{j} \in W$ with $w_{j}^{1} \in K, w_{j}^{2} \in L$ and $w_{j}^{3} \in T$,

$$
I_{i}\left(w_{j}\right)= \begin{cases}B_{b}\left(w_{j}^{1}\right) & , \text { if } w_{j} \in K-L-T, \\ y_{y}\left(w_{j}^{2}\right) & , \text { if } w_{j} \in L-K-T, \\ D_{d}\left(w_{j}^{3}\right) & , \text { if } w_{j} \in T-K-L, \\ Y_{y}\left(w_{j}^{2}\right) \sqcap_{R} D_{d}\left(w_{j}^{3}\right) & , \text { if } w_{j} \in L \cap T-K, \\ B_{b}\left(w_{j}^{1}\right) \sqcap_{R} Y_{y}\left(w_{j}^{2}\right) & , \text { if } w_{j} \in K \cap L-T, \\ B_{b}\left(w_{j}^{1}\right) \sqcap_{R} D_{d}\left(w_{j}^{3}\right) & , \text { if } w_{j} \in K \cap T-L, \\ B_{b}\left(w_{j}^{1}\right) \sqcap_{R} Y_{y}\left(w_{j}^{2}\right) \sqcap_{R} D_{d}\left(w_{j}^{3}\right) & , \text { if } w_{j} \in K \cap L \cap T .\end{cases}
$$

Then, $G_{g}\left(w_{j}\right)=I_{i}\left(w_{j}\right), \forall w_{j} \in W$. Hence, (9) is hwld and (10)-(12) are similar to (9). 
Proposition 4. Given that $\left(B_{b}, K, M\right)$ and $\left(Y_{y}, L, N\right)$ are any two PBIVNSS on $U$, then the following results hold:

(1) $\left(\left(B^{c}\right)_{\left(b^{c}\right)^{c}}^{c}, K, M\right)=\left(B_{b}, K, M\right)$,

(2) $\left(B_{b^{c}}^{c}, K, M\right) \sqcup_{E}\left(Y_{y^{c}}^{c}, L, N\right)=\left(\left(B_{b} \sqcap_{E} Y_{y}\right)^{c},(K \cup L), \max (M, N)\right)$,

(3) $\left(B_{b^{c}}^{c}, K, M\right) \sqcap_{E}\left(Y_{y^{c}}^{c}, L, N\right)=\left(\left(B_{b} \sqcup_{E} Y_{y}\right)^{c},(K \cup L), \max (M, N)\right)$,

(4) $\left(B_{b^{c}}^{c}, K, M\right) \sqcup_{R}\left(Y_{y^{c}}^{c}, L, N\right)=\left(\left(B_{b} \sqcap_{R} Y_{y}\right)^{c},(K \cap L), \max (M, N)\right)$,

(5) $\left(B_{b^{c}}^{c}, K, M\right) \sqcap_{R}\left(Y_{y^{c}}^{c}, L, N\right)=\left(\left(B_{b} \sqcup_{R} Y_{y}\right)^{c},(K \cap L), \min (M, N)\right)$.

Proof. (1) is straight-forward. We start from (2). Let $\left(B_{b}, K, M\right)$ and $\left(Y_{y}, L, N\right)$ (where $B_{b}: K \rightarrow B I^{U \times R} \times B I^{U \times R}$ and $\left.Y_{y}: L \rightarrow B I^{U \times R} \times B I^{U \times R}\right)$ be two PBIVNSS on $U$. Then by the definition of weak possibility belief interval-valued complement and extended union we have,

$$
\left(B_{b^{c}}^{c}, K, M\right) \sqcup_{E}\left(Y_{y^{c}}^{c}, L, N\right)=\left(J_{j}, S, P\right),
$$

where $J_{j}=B_{b^{c}}^{c} \sqcup_{E} Y_{y^{c}}^{c}, S=K \cup L, P=\max (M, N) ; \forall s_{j} \in S$ with $s_{j}^{1} \in K$ and $s_{j}^{2} \in L$,

$$
J_{j}\left(s_{j}\right)= \begin{cases}B_{b^{c}}^{c}\left(s_{j}^{1}\right) & , \text { if } s_{j} \in K-L, \\ Y_{y^{c}}^{c}\left(s_{j}^{2}\right) & , \text { if } s_{j} \in L-K, \\ B_{b^{c}}^{c}\left(s_{j}^{1}\right) \sqcup_{E} Y_{y^{c}}^{c}\left(s_{j}^{2}\right) & , \text { if } s_{j} \in K \cap L .\end{cases}
$$

Again by the definition of extended intersection we have $\left(F_{f}, S, P\right)=\left(B_{b} \cap_{E} Y_{y}\right), S=$ $K \cup L, P=\max (M, N) ; \forall s_{j} \in S$ with $s_{j}^{1} \in K$ and $s_{j}^{2} \in L$,

$$
F_{f}\left(s_{j}\right)= \begin{cases}B_{b}\left(s_{j}^{1}\right) & , \text { if } s_{j} \in K-L, \\ y_{y}\left(s_{j}^{2}\right) & , \text { if } s_{j} \in L-K, \\ B_{b}\left(s_{j}^{1}\right) \sqcap_{E} Y_{y}\left(s_{j}^{2}\right) & , \text { if } s_{j} \in K \cap L .\end{cases}
$$

Thus, by the definition of weak possibility belief interval-valued complement we have,

$$
F_{f^{c}}^{c}\left(s_{j}\right)= \begin{cases}B_{b^{c}}^{c}\left(s_{j}^{1}\right) & , \text { if } s_{j} \in K-L, \\ Y_{y^{c}}^{c}\left(s_{j}^{2}\right) & , \text { if } s_{j} \in L-K, \\ B_{b^{c}}^{c}\left(s_{j}^{1}\right) \sqcup_{E} Y_{y^{c}}^{c}\left(s_{j}^{2}\right) & , \text { if } s_{j} \in K \cap L .\end{cases}
$$

Then, $J_{j}\left(s_{j}\right)=F_{f^{c}}^{c}\left(s_{j}\right) ; \forall s_{j} \in S$. Hence, (2) holds. Remaining (3)-(5) are similar to (2).

In Figures 1 and 2, we give the flow charts of Algorithms 1 and 2 respectively. 


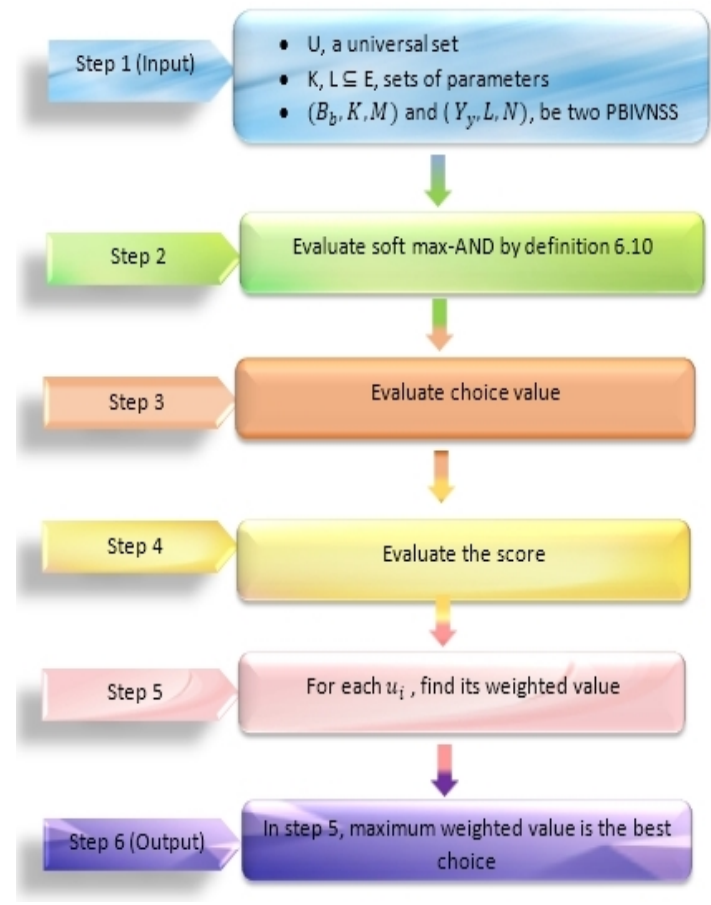

Figure 1. Flow chart of Algorithm 1.

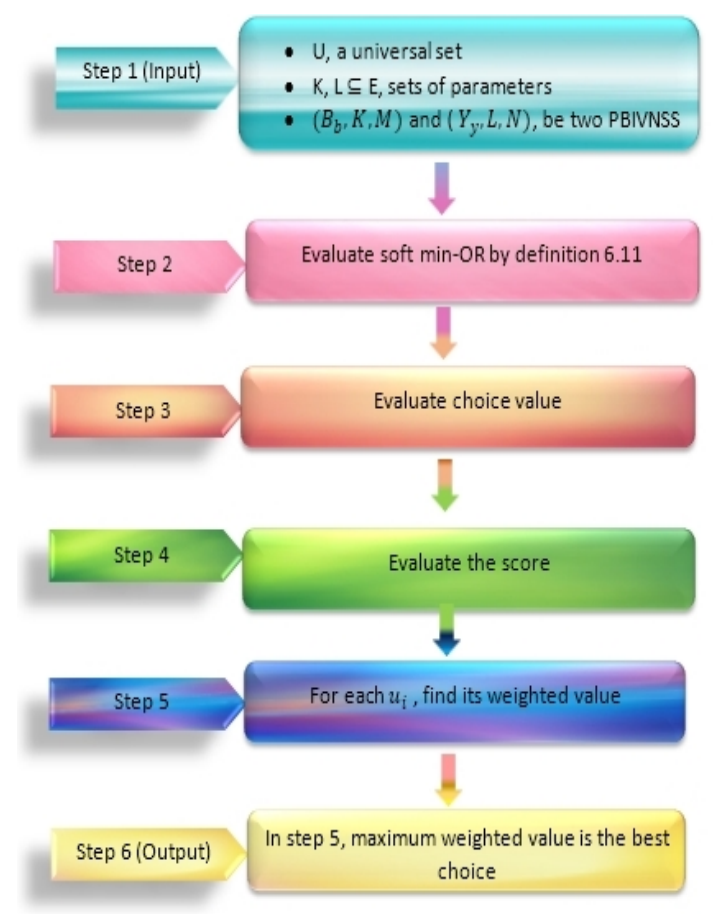

Figure 2. Flow chart of Algorithm 2.

\section{Algorithms}

In this section, we will present the algorithms on soft max-AND and soft min-OR operations on two possibility belief interval valued $\mathrm{N}$-soft sets for decision making. 


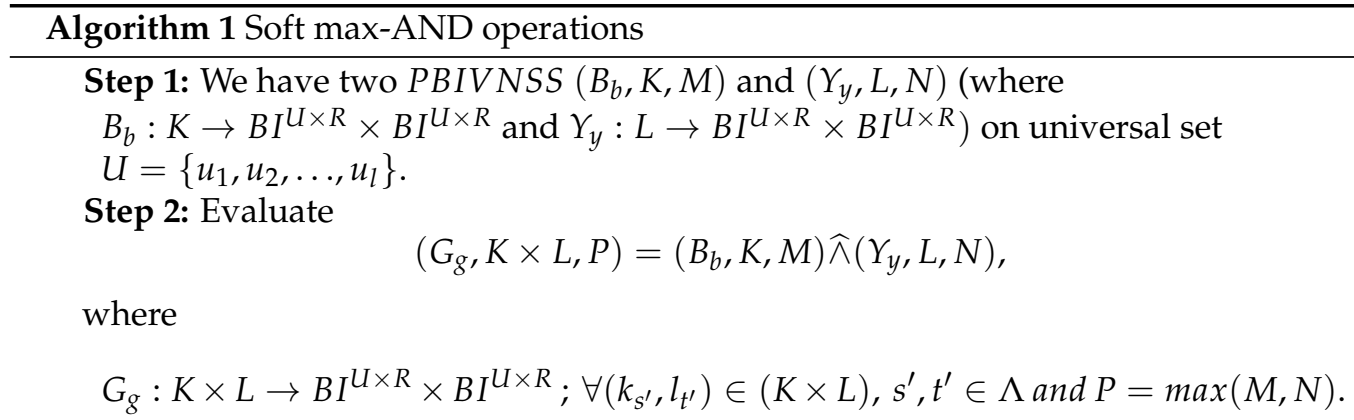

Step 3: Evaluate the choice value $\mathbb{C}\left(k_{s^{\prime}}, l_{t^{\prime}}\right)\left(u_{i}\right) ; \forall u_{i} \in U,\left(k_{s^{\prime}}, l_{t^{\prime}}\right) \in(K \times L), s^{\prime}, t^{\prime} \in \Lambda$ defined as:

$$
\mathbb{C}\left(k_{s^{\prime}}, l_{t^{\prime}}\right)\left(u_{i}\right)=\left\{\frac{r_{i\left(s^{\prime}, t^{\prime}\right)}}{I_{\mathbb{C}\left(k_{s^{\prime}}, l_{t^{\prime}}\right)}\left(u_{i}, r_{i\left(s^{\prime}, t^{\prime}\right)}\right)}\right\},
$$

where the interval of choice value is:

$$
\begin{aligned}
I_{\mathbb{C}\left(k_{s^{\prime}}, l_{t^{\prime}}\right)}\left(u_{i}, r_{i\left(s^{\prime}, t^{\prime}\right)}\right)=[ & B e l_{G\left(k_{s^{\prime}}, l_{t^{\prime}}\right)}\left(u_{i}, r_{i\left(s^{\prime}, t^{\prime}\right)}\right)+B e l_{g\left(k_{s^{\prime}}, l_{t^{\prime}}\right)}\left(u_{i}, r_{i\left(s^{\prime}, t^{\prime}\right)}\right), \\
& \left.P l_{G\left(k_{s^{\prime}}, l_{t^{\prime}}\right)}\left(u_{i}, r_{i\left(s^{\prime}, t^{\prime}\right)}\right)+P l_{g\left(k_{s^{\prime}}, l_{t^{\prime}}\right)}\left(u_{i}, r_{i\left(s^{\prime}, t^{\prime}\right)}\right)\right] .
\end{aligned}
$$

Step 4: Evaluate the score $\mathbb{S}\left(k_{s^{\prime}}, l_{t^{\prime}}\right)\left(u_{i}\right) ; \forall u_{i} \in U,\left(k_{s^{\prime}}, l_{t^{\prime}}\right) \in(K \times L), s^{\prime}, t^{\prime} \in \Lambda$ defined as:

$$
\mathbb{S}\left(k_{s^{\prime}}, l_{t^{\prime}}\right)\left(u_{i}\right)=\mathbb{S}_{1}\left(k_{s^{\prime}}, l_{t^{\prime}}\right)\left(u_{i}\right) \times \mathbb{S}_{2}\left(k_{s^{\prime}}, l_{t^{\prime}}\right)\left(u_{i}\right),
$$

where,

$$
\begin{array}{r}
\mathbb{S}_{1}\left(k_{s^{\prime}}, l_{t^{\prime}}\right)\left(u_{i}\right)=\sum_{t_{q} \in U}[ \\
{\left[\alpha_{1} I_{\mathbb{C}\left(k_{s^{\prime}}, l_{t^{\prime}}\right)}\left(u_{i}, r_{i\left(s^{\prime}, t^{\prime}\right)}\right)-\alpha_{1} I_{\mathbb{C}\left(k_{s^{\prime}}, l_{t^{\prime}}\right)}\left(t_{q}, r_{q\left(s^{\prime}, t^{\prime}\right)}\right)+\right.} \\
\left.\alpha_{2} I_{\mathbb{C}\left(k_{s^{\prime}}, l_{t^{\prime}}\right)}\left(u_{i}, r_{i\left(s^{\prime}, t^{\prime}\right)}\right)-\alpha_{2} I_{\mathbb{C}\left(k_{s^{\prime}}, l_{t^{\prime}}\right)}\left(t_{q}, r_{q\left(s^{\prime}, t^{\prime}\right)}\right)\right] .
\end{array}
$$

where $\alpha_{m}:[0,1] \rightarrow[0,1]$ is the $m$ th projection mapping such that $\alpha_{1}$ is the lower membership value and $\alpha_{2}$ is the upper membership value of choice interval and

$$
\mathbb{S}_{2}\left(k_{s^{\prime}}, l_{t^{\prime}}\right)\left(u_{i}\right)=\sum_{q=1}^{l} \max \left\{r_{i\left(s^{\prime}, t^{\prime}\right)}, r_{q\left(s^{\prime}, t^{\prime}\right)}\right\} .
$$

Step 5: For each $\mathbb{S}\left(u_{l}\right)$ it's weighted value is:

$$
\mathbb{S}\left(u_{l}\right)=\sum_{\left(k_{s^{\prime}}, l_{l^{\prime}}\right) \in(K \times L)} \mathbb{S}\left(k_{s^{\prime}}, l_{t^{\prime}}\right)\left(u_{l}\right) .
$$

Step 6: Now the optimal decision is:

$$
\mathfrak{X}=\arg \max \left\{\mathbb{S}\left(u_{1}\right), \mathbb{S}\left(u_{2}\right), \text { cdots, } \mathbb{S}\left(u_{l}\right)\right\} .
$$




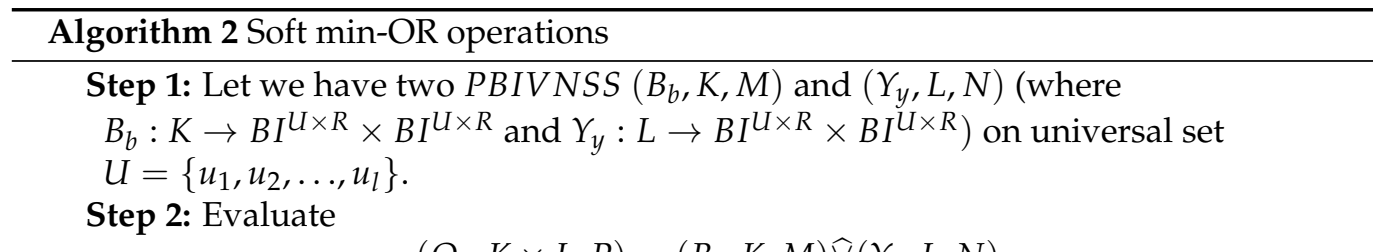

$$
\left(Q_{q}, K \times L, P\right)=\left(B_{b}, K, M\right) \widehat{V}\left(Y_{y}, L, N\right),
$$

where

$$
Q_{q}: K \times L \rightarrow B I^{U \times R} \times B I^{U \times R} ; \forall\left(k_{s^{\prime}}, l_{t^{\prime}}\right) \in(K \times L),
$$

$s^{\prime}, t^{\prime} \in \Lambda$ and $O=\min (M, N)$.

Step 3: Evaluate the choice value

$\mathbb{C}\left(k_{s^{\prime}}, l_{t^{\prime}}\right)\left(u_{i}\right) ; \forall u_{i} \in U,\left(k_{s^{\prime}}, l_{t^{\prime}}\right) \in(K \times L), s^{\prime}, t^{\prime} \in \Lambda$ defined as:

$$
\mathbb{C}\left(k_{s^{\prime}}, l_{t^{\prime}}\right)\left(u_{i}\right)=\left\{\frac{r_{i\left(s^{\prime}, t^{\prime}\right)}}{I_{\mathbb{C}\left(k_{s^{\prime}}, l_{t^{\prime}}\right)}\left(u_{i}, r_{i\left(s^{\prime}, t^{\prime}\right)}\right)}\right\},
$$

where the interval of choice value is:

$$
\begin{aligned}
I_{\mathbb{C}\left(k_{s^{\prime}}, l_{t^{\prime}}\right)}\left(u_{i}, r_{i\left(s^{\prime}, t^{\prime}\right)}\right)=[ & \operatorname{Bel}_{G\left(k_{s^{\prime}}, l_{t^{\prime}}\right)}\left(u_{i}, r_{i\left(s^{\prime}, t^{\prime}\right)}\right)+B e l_{g\left(k_{s^{\prime}}, l_{t^{\prime}}\right)}\left(u_{i}, r_{i\left(s^{\prime}, t^{\prime}\right)}\right), \\
& \left.P l_{G\left(k_{s^{\prime}}, l_{t^{\prime}}\right)}\left(u_{i}, r_{i\left(s^{\prime}, t^{\prime}\right)}\right)+P l_{g\left(k_{s^{\prime}}, l_{t^{\prime}}\right)}\left(u_{i}, r_{i\left(s^{\prime}, t^{\prime}\right)}\right)\right] .
\end{aligned}
$$

Step 4: Evaluate the score $\mathbb{S}\left(k_{s^{\prime}}, l_{t^{\prime}}\right)\left(u_{i}\right) ; \forall u_{i} \in U,\left(k_{s^{\prime}}, l_{t^{\prime}}\right) \in(K \times L), s^{\prime}, t^{\prime} \in \Lambda$ defined as:

$$
\mathbb{S}\left(k_{s^{\prime}}, l_{t^{\prime}}\right)\left(u_{i}\right)=\mathbb{S}_{1}\left(k_{s^{\prime}}, l_{t^{\prime}}\right)\left(u_{i}\right) \times \mathbb{S}_{2}\left(k_{s^{\prime}}, l_{t^{\prime}}\right)\left(u_{i}\right),
$$

where,

$$
\begin{array}{r}
\mathbb{S}_{1}\left(k_{s^{\prime}}, l_{t^{\prime}}\right)\left(u_{i}\right)=\sum_{t_{q} \in U}[ \\
{\left[\alpha_{1} I_{\mathbb{C}\left(k_{s^{\prime}}, l_{t^{\prime}}\right)}\left(u_{i}, r_{i\left(s^{\prime}, t^{\prime}\right)}\right)-\alpha_{1} I_{\mathbb{C}\left(k_{s^{\prime}}, l_{t^{\prime}}\right)}\left(t_{q}, r_{q\left(s^{\prime}, t^{\prime}\right)}\right)+\right.} \\
\left.\alpha_{2} I_{\mathbb{C}\left(k_{s^{\prime}}, l_{t^{\prime}}\right)}\left(u_{i}, r_{i\left(s^{\prime}, t^{\prime}\right)}\right)-\alpha_{2} I_{\mathbb{C}\left(k_{s^{\prime}}, l_{t^{\prime}}\right)}\left(t_{q}, r_{q\left(s^{\prime}, t^{\prime}\right)}\right)\right],
\end{array}
$$

where $\alpha_{m}:[0,1] \rightarrow[0,1]$ is the $m$ th projection mapping such that $\alpha_{1}$ is the lower membership value and $\alpha_{2}$ is the upper membership value of choice interval and

$$
\mathbb{S}_{2}\left(k_{s^{\prime}}, l_{t^{\prime}}\right)\left(u_{i}\right)=\sum_{q=1}^{l} \max \left\{r_{i\left(s^{\prime}, t^{\prime}\right)}, r_{q\left(s^{\prime}, t^{\prime}\right)}\right\}
$$

Step 5: For each $\mathbb{S}\left(u_{l}\right)$ it's weighted value is:

$$
\mathbb{S}\left(u_{l}\right)=\sum_{\left(k_{s^{\prime}}, l_{l^{\prime}}\right) \in(K \times L)} \mathbb{S}\left(k_{s^{\prime}}, l_{t^{\prime}}\right)\left(u_{l}\right) .
$$

Step 6: Now the optimal decision is:

$$
\mathfrak{X}=\arg \max \left\{\mathbb{S}\left(u_{1}\right), \mathbb{S}\left(u_{2}\right), \ldots, \mathbb{S}\left(u_{l}\right)\right\} .
$$

\section{Applications}

In this section, we give the application of our proposed sets.

Example 11. Let $M r$. $\mathcal{H}$ wants to buy a smartphone with particular features, and there are three smartphones that are under consideration. Let $U=\left\{u_{1}, u_{2}, u_{3}, u_{4}, u_{5}, u_{6}\right\}$ be the set of smartphones. Let $E$ be the set of parameters (particular features) for the evaluation of smartphone and $K, L \subseteq E$ such that $K=\left\{k_{1}=\right.$ Reasonable, $k_{3}=$ Expensive $\}$ and $L=\left\{k_{4}=\right.$ High resolution, $k_{5}=$ Good battery timing, $k_{6}=$ Guest mode $\}$. Assume that Mr. H wants to 
buy one such smartphone depending on two parameters only. Suppose there are two observations $\left(B_{b}, K, 4\right)$ and $\left(Y_{y}, L, 7\right)$ by two experts as follows:

$$
\begin{aligned}
& \left(B_{b}, K, 4\right)=\left\{\left(k_{1},\left(\frac{\left(u_{1}, 1\right)}{[0.1,0.6],[0.4,0.7]^{\prime}}, \frac{\left(u_{2}, 3\right)}{[0.8,0.9],[0.3,0.8]^{\prime}}, \frac{\left(u_{3}, 1\right)}{[0.2,0.4],[0.2,0.7]^{\prime}},\right.\right.\right. \\
& \left.\left.\frac{\left(u_{4}, 2\right)}{[0.7,0.8],[0.5,0.6]}, \frac{\left(u_{5}, 0\right)}{[0.5,0.9],[0.7,0.9]}, \frac{\left(u_{6}, 3\right)}{[0.6,0.8],[0.4,0.6]}\right)\right) \text {, } \\
& \left(k_{3},\left(\frac{\left(u_{1}, 0\right)}{[0.5,0.6],[0.2,0.8]}, \frac{\left(u_{2}, 3\right)}{[0.4,0.8],[0.7,0.9]}, \frac{\left(u_{3}, 2\right)}{[0.3,0.4],[0.4,0.5]},\right.\right. \\
& \left.\left.\left.\frac{\left(u_{4}, 2\right)}{[0.2,0.3],[0.5,0.7]}, \frac{\left(u_{5}, 1\right)}{[0.3,0.7],[0.4,0.8]}, \frac{\left(u_{6}, 1\right)}{[0.9,1.0],[0.1,0.7]}\right)\right)\right\} \text {. } \\
& \left(Y_{y}, L, 7\right)=\left\{\left(k_{4},\left(\frac{\left(u_{1}, 3\right)}{[0.6,0.9],[0.1,0.4]}, \frac{\left(u_{2}, 1\right)}{[0.2,0.9],[0.4,0.6]}, \frac{\left(u_{3}, 4\right)}{[0.7,0.8],[0.1,0.2]},\right.\right.\right. \\
& \left.\left.\frac{\left(u_{4}, 1\right)}{[0.2,0.9],[0.3,0.5]}, \frac{\left(u_{5}, 6\right)}{[0.4,0.6],[0.8,1.0]}, \frac{\left(u_{6}, 0\right)}{[0.1,0.9],[0.3,0.8]}\right)\right), \\
& \left(k_{5},\left(\frac{\left(u_{1}, 0\right)}{[0.1,0.7],[0.3,0.9]}, \frac{\left(u_{2}, 2\right)}{[0.7,0.9],[0.6,0.8]}, \frac{\left(u_{3}, 6\right)}{[0.5,0.9],[0.1,0.5]},\right.\right. \\
& \left(u_{4}, 5\right) \quad\left(u_{5}, 1\right) \quad\left(u_{6}, 3\right) \\
& \overline{[0.6,1.0],[0.4,0.5]}, \overline{[0.7,0.8],[0.3,0.4]}, \overline{[0.6,0.7],[0.6,0.8]})) \text {, } \\
& \left(k_{6},\left(\frac{\left(u_{1}, 1\right)}{[0.2,0.8],[0.3,0.4]}, \frac{\left(u_{2}, 5\right)}{[0.8,0.9],[0.2,0.3]}, \frac{\left(u_{3}, 0\right)}{[0.6,0.8],[0.4,0.8]}\right. \text {, }\right. \\
& \left.\left.\left.\frac{\left(u_{4}, 6\right)}{[0.1,0.4],[0.2,0.3]}, \frac{\left(u_{5}, 4\right)}{[0.6,0.7],[0.5,0.6]}, \frac{\left(u_{6}, 6\right)}{[0.2,0.9],[0.4,0.7]}\right)\right)\right\} \text {. }
\end{aligned}
$$

Firstly, we will evaluate the soft max-AND operation $\left(G_{g}, K \times L, 7\right)=\left(B_{b}, K, 4\right) \widehat{\wedge}\left(Y_{y}, L, 7\right)$ by using step 2 of Algorithm 1:

$$
\begin{aligned}
& \left(G_{g}, K \times L, 7\right)=\left\{\left(\left(k_{1}, k_{4}\right),\left(\frac{\left(u_{1}, 3\right)}{[0.26,0.70],[0.20,0.50]}, \frac{\left(u_{2}, 3\right)}{[0.40,0.90],[0.33,0.66]}, \frac{\left(u_{3}, 4\right)}{[0.36,0.53],[0.13,0.36]},\right.\right.\right. \\
& \left.\left.\frac{\left(u_{4}, 2\right)}{,}, \frac{\left(u_{5}, 6\right)}{[0.43,0.70],[0.73,0.93]}, \frac{\left(u_{6}, 3\right)}{[0.26,0.83],[0.33,0.66]}\right)\right) \\
& \left(\left(k_{1}, k_{5}\right),\left(\frac{\left(u_{1}, 1\right)}{[0.10,0.63],[0.33,0.76]}, \frac{\left(u_{2}, 3\right)}{[0.73,0.90],[0.40,0.80]}, \frac{\left(u_{3}, 6\right)}{[0.30,0.56],[0.13,0.56]},\right.\right. \\
& \left.\left.\frac{\left(u_{4}, 5\right)}{[0.63,0.86],[0.43,0.53]}, \frac{\left(u_{5}, 1\right)}{[0.56,0.83],[0.43,0.56]}, \frac{\left(u_{6}, 3\right)}{[0.60,0.73],[0.46,0.66]}\right)\right), \\
& \left(\left(k_{1}, k_{6}\right),\left(\frac{\left(u_{1}, 1\right)}{[0.13,0.66],[0.33,0.50]}, \frac{\left(u_{2}, 5\right)}{[0.80,0.90],[0.23,0.46]}, \frac{\left(u_{3}, 1\right)}{[0.33,0.53],[0.26,0.73]},\right.\right. \\
& \left.\left.\frac{\left(u_{4}, 6\right)}{[0.30,0.53],[0.30,0.40]}, \frac{\left(u_{5}, 4\right)}{[0.53,0.76],[0.56,0.70]}, \frac{\left(u_{6}, 6\right)}{[0.33,0.83],[0.40,0.63]}\right)\right), \\
& \left(\left(k_{3}, k_{4}\right),\left(\frac{\left(u_{1}, 3\right)}{[0.53,0.70],[0.13,0.53]}, \frac{\left(u_{2}, 3\right)}{[0.26,0.83],[0.50,0.70]}, \frac{\left(u_{3}, 4\right)}{[0.43,0.53],[0.20,0.30]},\right.\right. \\
& \left.\left.\frac{\left(u_{4}, 2\right)}{0.50],[0.36,0.56]}, \frac{\left(u_{5}, 6\right)}{[0.33,0.63],[0.53,0.86]}, \frac{\left(u_{6}, 1\right)}{[0.36,0.93],[0.16,0.73]}\right)\right) \\
& \left(\left(k_{3}, k_{5}\right),\left(\frac{\left(u_{1}, 0\right)}{[0.23,0.63],[0.23,0.83]}, \frac{\left(u_{2}, 3\right)}{[0.50,0.83],[0.63,0.83]}, \frac{\left(u_{3}, 6\right)}{[0.36,0.56],[0.20,0.50]},\right.\right. \\
& \left.\left.\frac{\left(u_{4}, 5\right)}{[0.33,0.53],[0.43,0.56]}, \frac{\left(u_{5}, 1\right)}{[0.43,0.73],[0.33,0.53]}, \frac{\left(u_{6}, 3\right)}{[0.70,0.80],[0.26,0.73]}\right)\right), \\
& \left(\left(k_{3}, k_{6}\right),\left(\frac{\left(u_{1}, 1\right)}{[0.30,0.66],[0.23,0.53]}, \frac{\left(u_{2}, 5\right)}{[0.53,0.83],[0.36,0.50]}, \frac{\left(u_{3}, 2\right)}{[0.40,0.53],[0.40,0.60]},\right.\right. \\
& \left.\left.\left.\frac{\left(u_{4}, 6\right)}{[0.13,0.33],[0.30,0.43]}, \frac{\left(u_{5}, 4\right)}{[0.40,0.70],[0.43,0.66]}, \frac{\left(u_{6}, 6\right)}{[0.43,0.93],[0.20,0.70]}\right)\right)\right\} .
\end{aligned}
$$

Then we will evaluate the choice value $\mathbb{C}\left(k_{s^{\prime}}, l_{t^{\prime}}\right)\left(u_{i}\right) ; \forall u_{i} \in U,\left(k_{s^{\prime}}, l_{t^{\prime}}\right) \in(K \times L), s^{\prime}=$ 1,3 and $t^{\prime}=4,5,6$ by using step 3 of Algorithm 1: 


\begin{tabular}{lclc}
\hline $\mathbb{C}\left(\boldsymbol{k}_{\boldsymbol{s}^{\prime}}, \boldsymbol{k}_{\boldsymbol{t}^{\prime}}\right)\left(u_{\boldsymbol{i}}\right)$ & $($ Grading, Interval Value $)$ & $\mathbb{C}\left(\boldsymbol{k}_{\boldsymbol{s}^{\prime}}, \boldsymbol{k}_{\boldsymbol{t}^{\prime}}\right)\left(u_{\boldsymbol{i}}\right)$ & (Grading, Interval Value $)$ \\
\hline $\mathbb{C}\left(k_{1}, k_{4}\right)\left(u_{1}\right)$ & $(3,[0.46,1.20])$ & $\mathbb{C}\left(k_{3}, k_{4}\right)\left(u_{1}\right)$ & $(3,[0.66,1.23])$ \\
\hline $\mathbb{C}\left(k_{1}, k_{4}\right)\left(u_{2}\right)$ & $(3,[0.73,1.56])$ & $\mathbb{C}\left(k_{3}, k_{4}\right)\left(u_{2}\right)$ & $(3,[0.76,1.53])$ \\
\hline $\mathbb{C}\left(k_{1}, k_{4}\right)\left(u_{3}\right)$ & $(4,[0.50,0.90])$ & $\mathbb{C}\left(k_{3}, k_{4}\right)\left(u_{3}\right)$ & $(4,[0.63,0.83])$ \\
\hline $\mathbb{C}\left(k_{1}, k_{4}\right)\left(u_{4}\right)$ & $(2,[0.73,1.36])$ & $\mathbb{C}\left(k_{3}, k_{4}\right)\left(u_{4}\right)$ & $(2,[0.56,1.06])$ \\
\hline $\mathbb{C}\left(k_{1}, k_{4}\right)\left(u_{5}\right)$ & $(6,[1.16,1.63])$ & $\mathbb{C}\left(k_{3}, k_{4}\right)\left(u_{5}\right)$ & $(6,[0.86,1.50])$ \\
\hline $\mathbb{C}\left(k_{1}, k_{4}\right)\left(u_{6}\right)$ & $(3,[0.60,1.50])$ & $\mathbb{C}\left(k_{3}, k_{4}\right)\left(u_{6}\right)$ & $(1,[0.53,1.66])$ \\
\hline $\mathbb{C}\left(k_{1}, k_{5}\right)\left(u_{1}\right)$ & $(1,[0.43,1.40])$ & $\mathbb{C}\left(k_{3}, k_{5}\right)\left(u_{1}\right)$ & $(0,[0.46,1.46])$ \\
\hline $\mathbb{C}\left(k_{1}, k_{5}\right)\left(u_{2}\right)$ & $(3,[1.13,1.70])$ & $\mathbb{C}\left(k_{3}, k_{5}\right)\left(u_{2}\right)$ & $(3,[1.13,1.66])$ \\
\hline $\mathbb{C}\left(k_{1}, k_{5}\right)\left(u_{3}\right)$ & $(6,[0.43,1.13])$ & $\mathbb{C}\left(k_{3}, k_{5}\right)\left(u_{3}\right)$ & $(6,[0.56,1.06])$ \\
\hline $\mathbb{C}\left(k_{1}, k_{5}\right)\left(u_{4}\right)$ & $(5,[1.06,1.40])$ & $\mathbb{C}\left(k_{3}, k_{5}\right)\left(u_{4}\right)$ & $(5,[0.76,1.10])$ \\
\hline $\mathbb{C}\left(k_{1}, k_{5}\right)\left(u_{5}\right)$ & $(1,[1.00,1.40])$ & $\mathbb{C}\left(k_{3}, k_{5}\right)\left(u_{5}\right)$ & $(1,[0.76,1.26])$ \\
\hline $\mathbb{C}\left(k_{1}, k_{5}\right)\left(u_{6}\right)$ & $(3,[1.06,1.40])$ & $\mathbb{C}\left(k_{3}, k_{5}\right)\left(u_{6}\right)$ & $(3,[0.96,1.53])$ \\
\hline $\mathbb{C}\left(k_{1}, k_{6}\right)\left(u_{1}\right)$ & $(1,[0.46,1.16])$ & $\mathbb{C}\left(k_{3}, k_{6}\right)\left(u_{1}\right)$ & $(1,[0.53,1.20])$ \\
\hline $\mathbb{C}\left(k_{1}, k_{6}\right)\left(u_{2}\right)$ & $(5,[1.03,1.36])$ & $\mathbb{C}\left(k_{3}, k_{6}\right)\left(u_{2}\right)$ & $(5,[0.90,1.33])$ \\
\hline $\mathbb{C}\left(k_{1}, k_{6}\right)\left(u_{3}\right)$ & $(1,[0.60,1.26])$ & $\mathbb{C}\left(k_{3}, k_{6}\right)\left(u_{3}\right)$ & $(2,[0.80,1.13])$ \\
\hline $\mathbb{C}\left(k_{1}, k_{6}\right)\left(u_{4}\right)$ & $(6,[0.60,0.93])$ & $\mathbb{C}\left(k_{3}, k_{6}\right)\left(u_{4}\right)$ & $(6,[0.43,0.76])$ \\
\hline $\mathbb{C}\left(k_{1}, k_{6}\right)\left(u_{5}\right)$ & $(4,[1.10,1.46])$ & $\mathbb{C}\left(k_{3}, k_{6}\right)\left(u_{5}\right)$ & $(4,[0.83,1.36])$ \\
\hline $\mathbb{C}\left(k_{1}, k_{6}\right)\left(u_{6}\right)$ & $(6,[0.73,1.46])$ & $\mathbb{C}\left(k_{3}, k_{6}\right)\left(u_{6}\right)$ & $(6,[0.63,1.63])$ \\
\hline & & & \\
& & & \\
\hline
\end{tabular}

Now we will evaluate the Score $\mathbb{S}\left(k_{s^{\prime}}, l_{t^{\prime}}\right)\left(u_{i}\right) ; \forall u_{i} \in U,\left(k_{s^{\prime}}, l_{t^{\prime}}\right) \in(K \times L), \quad s^{\prime}=$ 1,3 and $t^{\prime}=4,5,6$ by using step 4 of Algorithm 1 .

\begin{tabular}{lccc}
\hline $\mathbb{S}\left(k_{s^{\prime}}, k_{t^{\prime}}\right)\left(u_{i}\right)$ & The Score & $\mathbb{S}\left(k_{s^{\prime}}, k_{t^{\prime}}\right)\left(u_{i}\right)$ & The Score \\
\hline $\mathbb{S}\left(k_{1}, k_{4}\right)\left(u_{1}\right)$ & -52.14 & $\mathbb{S}\left(k_{3}, k_{4}\right)\left(u_{1}\right)$ & -10.34 \\
\hline $\mathbb{S}\left(k_{1}, k_{4}\right)\left(u_{2}\right)$ & 31.02 & $\mathbb{S}\left(k_{3}, k_{4}\right)\left(u_{2}\right)$ & 42.46 \\
\hline $\mathbb{S}\left(k_{1}, k_{4}\right)\left(u_{3}\right)$ & -102.18 & $\mathbb{S}\left(k_{3}, k_{4}\right)\left(u_{3}\right)$ & -79.3 \\
\hline $\mathbb{S}\left(k_{1}, k_{4}\right)\left(u_{4}\right)$ & 4.41 & $\mathbb{S}\left(k_{3}, k_{4}\right)\left(u_{4}\right)$ & -41.8 \\
\hline $\mathbb{S}\left(k_{1}, k_{4}\right)\left(u_{5}\right)$ & 158.76 & $\mathbb{S}\left(k_{3}, k_{4}\right)\left(u_{5}\right)$ & 84.6 \\
\hline $\mathbb{S}\left(k_{1}, k_{4}\right)\left(u_{6}\right)$ & 5.94 & $\mathbb{S}\left(k_{3}, k_{4}\right)\left(u_{6}\right)$ & 25.27 \\
\hline $\mathbb{S}\left(k_{1}, k_{5}\right)\left(u_{1}\right)$ & -48.64 & $\mathbb{S}\left(k_{3}, k_{5}\right)\left(u_{1}\right)$ & -21.24 \\
\hline $\mathbb{S}\left(k_{1}, k_{5}\right)\left(u_{2}\right)$ & 79.12 & $\mathbb{S}\left(k_{3}, k_{5}\right)\left(u_{2}\right)$ & 92.92 \\
\hline $\mathbb{S}\left(k_{1}, k_{5}\right)\left(u_{3}\right)$ & -150.48 & $\mathbb{S}\left(k_{3}, k_{5}\right)\left(u_{3}\right)$ & -107.28 \\
\hline $\mathbb{S}\left(k_{1}, k_{5}\right)\left(u_{4}\right)$ & 37.82 & $\mathbb{S}\left(k_{3}, k_{5}\right)\left(u_{4}\right)$ & -47.74 \\
\hline $\mathbb{S}\left(k_{1}, k_{5}\right)\left(u_{5}\right)$ & $\mathbb{S}\left(k_{3}, k_{5}\right)\left(u_{5}\right)$ & -11.02 \\
\hline $\mathbb{S}\left(k_{1}, k_{5}\right)\left(u_{6}\right)$ & 16.34 & $\mathbb{S}\left(k_{3}, k_{5}\right)\left(u_{6}\right)$ & 51.52 \\
\hline $\mathbb{S}\left(k_{1}, k_{6}\right)\left(u_{1}\right)$ & 28.06 & $\mathbb{S}\left(k_{3}, k_{6}\right)\left(u_{1}\right)$ & -27.6 \\
\hline $\mathbb{S}\left(k_{1}, k_{6}\right)\left(u_{2}\right)$ & -55.89 & $\mathbb{S}\left(k_{3}, k_{6}\right)\left(u_{2}\right)$ & 59.2 \\
\hline
\end{tabular}




\begin{tabular}{lccc}
\hline $\mathbb{S}\left(k_{s^{\prime}}, k_{t^{\prime}}\right)\left(u_{i}\right)$ & The Score & $\mathbb{S}\left(k_{s^{\prime}}, k_{t^{\prime}}\right)\left(u_{i}\right)$ & The Score \\
\hline $\mathbb{S}\left(k_{1}, k_{6}\right)\left(u_{3}\right)$ & -22.77 & $\mathbb{S}\left(k_{3}, k_{6}\right)\left(u_{3}\right)$ & 1.25 \\
\hline $\mathbb{S}\left(k_{1}, k_{6}\right)\left(u_{4}\right)$ & -106.92 & $\mathbb{S}\left(k_{3}, k_{6}\right)\left(u_{4}\right)$ & -158.04 \\
\hline $\mathbb{S}\left(k_{1}, k_{6}\right)\left(u_{5}\right)$ & 93.09 & $\mathbb{S}\left(k_{3}, k_{6}\right)\left(u_{5}\right)$ & 46.69 \\
\hline $\mathbb{S}\left(k_{1}, k_{6}\right)\left(u_{6}\right)$ & 35.64 & $\mathbb{S}\left(k_{3}, k_{6}\right)\left(u_{6}\right)$ & 73.08 \\
\hline
\end{tabular}

By using step 5 of Algorithm 1 the weighted values for each $\mathbb{S}\left(u_{l}\right)$ are:

$$
\begin{aligned}
& \mathbb{S}\left(u_{1}\right)=-215.85, \mathbb{S}\left(u_{2}\right)=374.8, \mathbb{S}\left(u_{3}\right)=-460.76, \\
& \mathbb{S}\left(u_{4}\right)=-312.27, \mathbb{S}\left(u_{5}\right)=388.46, \mathbb{S}\left(u_{6}\right)=219.51 .
\end{aligned}
$$

Here the optimal decision by using step 6 of Algorithm 1 is:

$$
\mathfrak{X}=\arg \max \left\{\mathbb{S}\left(u_{1}\right), \mathbb{S}\left(u_{2}\right), \mathbb{S}\left(u_{3}\right), \mathbb{S}\left(u_{4}\right), \mathbb{S}\left(u_{5}\right), \mathbb{S}\left(u_{6}\right)\right\} .
$$

Thus, $u_{5}$ is the best choice. Hence Mr. $\mathcal{H}$ will buy the $u_{5}$ smartphone.

In Figures 3 and 4, we give the graphical behavior of score values of Examples 11 and 12 by means of Algorithms 1 and 2 respectively.

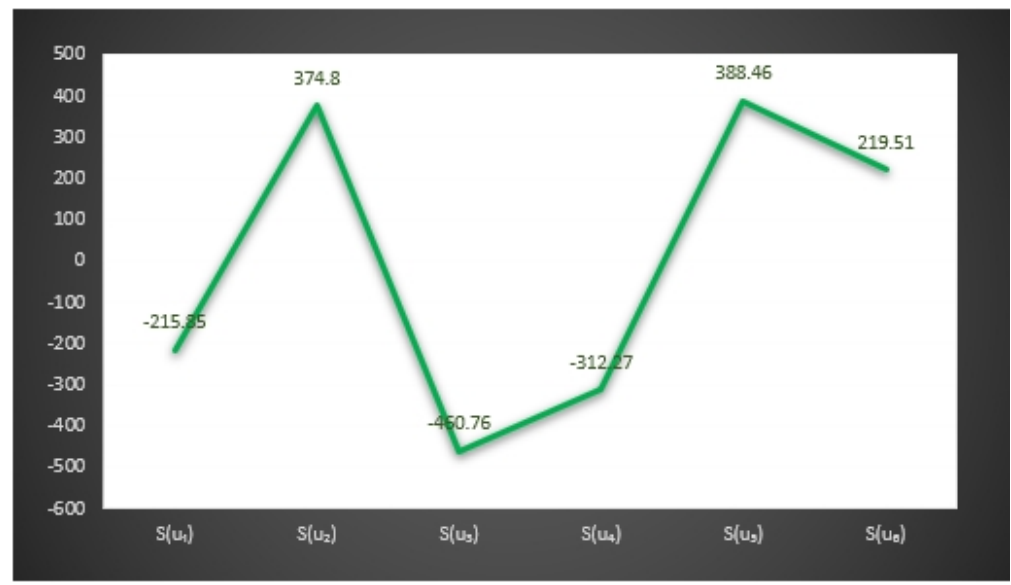

Figure 3. Graphical behavior of score values of Example 11 by means of Algorithm 1.

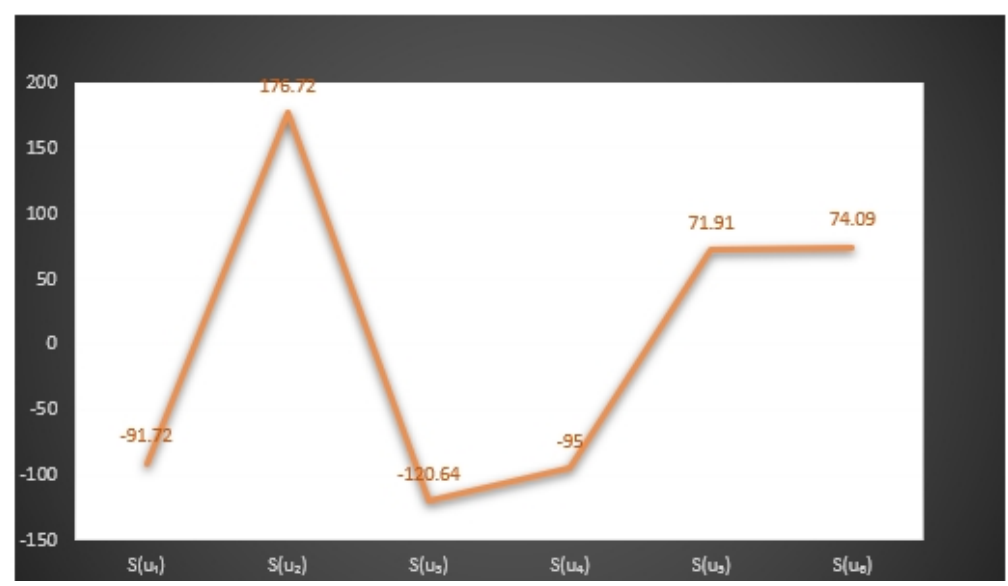

Figure 4. Graphical behavior of score values of Example 12 by means of Algorithm 2.

Example 12. Consider the Example 11 and assume that Mr. H wants to buy one such smartphone depending on one of two parameters only. 
Firstly, we will evaluate the soft min-OR operation $\left(Q_{q}, K \times L, 4\right)=\left(B_{b}, K, 4\right) \widehat{\nabla}\left(Y_{y}, L, 7\right)$ by using step 2 of Algorithm 2:

$$
\begin{aligned}
& \left(Q_{q}, K \times L, 4\right)=\left\{\left(\left(k_{1}, k_{4}\right),\left(\frac{\left(u_{1}, 1\right)}{[0.43,0.80],[0.30,0.60]}, \frac{\left(u_{2}, 1\right)}{[0.60,0.90],[0.36,0.73]}, \frac{\left(u_{3}, 1\right)}{[0.53,0.66],[0.16,0.53]},\right.\right.\right. \\
& \left.\left.\frac{\left(u_{4}, 1\right)}{[0.53,0.86],[0.43,0.56]}, \frac{\left(u_{5}, 0\right)}{[0.46,0.80],[0.76,0.96]}, \frac{\left(u_{6}, 0\right)}{[0.43,0.86],[0.36,0.73]}\right)\right), \\
& \left(\left(k_{1}, k_{5}\right),\left(\frac{\left(u_{1}, 0\right)}{[0.10,0.66],[0.36,0.83]}, \frac{\left(u_{2}, 2\right)}{[0.76,0.90],[0.50,0.80]}, \frac{\left(u_{3}, 1\right)}{[0.40,0.73],[0.16,0.63]},\right.\right. \\
& \left.\left.\frac{\left(u_{4}, 2\right)}{[0.66,0.93],[0.46,0.56]}, \frac{\left(u_{5}, 0\right)}{[0.63,0.86],[0.56,0.73]}, \frac{\left(u_{6}, 3\right)}{[0.60,0.76],[0.53,0.73]}\right)\right), \\
& \left(\left(k_{1}, k_{6}\right),\left(\frac{\left(u_{1}, 1\right)}{[0.16,0.73],[0.36,0.60]}, \frac{\left(u_{2}, 3\right)}{[0.80,0.90],[0.26,0.63]}, \frac{\left(u_{3}, 0\right)}{[0.46,0.66],[0.33,0.76]},\right.\right. \\
& \left.\left.\frac{\left(u_{4}, 2\right)}{0.66],[0.40,0.50]}, \frac{\left(u_{5}, 0\right)}{[0.56,0.83],[0.63,0.80]}, \frac{\left(u_{6}, 3\right)}{[0.46,0.86],[0.40,0.66]}\right)\right) \text {, } \\
& \left(\left(k_{3}, k_{4}\right),\left(\frac{\left(u_{1}, 0\right)}{[0.56,0.80],[0.16,0.66]}, \frac{\left(u_{2}, 1\right)}{[0.33,0.86],[0.60,0.80]}, \frac{\left(u_{3}, 2\right)}{[0.56,0.66],[0.30,0.40]},\right.\right. \\
& \left.\left.\frac{\left(u_{4}, 1\right)}{0.70],[0.43,0.63]}, \frac{\left(u_{5}, 1\right)}{[0.36,0.66],[0.66,0.93]}, \frac{\left(u_{6}, 0\right)}{[0.63,0.96],[0.23,0.76]}\right)\right), \\
& \left(\left(k_{3}, k_{5}\right),\left(\frac{\left(u_{1}, 0\right)}{[0.36,0.66],[0.26,0.86]}, \frac{\left(u_{2}, 2\right)}{[0.60,0.86],[0.66,0.86]}, \frac{\left(u_{3}, 2\right)}{[0.43,0.73],[0.30,0.50]},\right.\right. \\
& \left.\left.\frac{\left(u_{4}, 2\right)}{0.76],[0.46,0.63]}, \frac{\left(u_{5}, 1\right)}{[0.56,0.76],[0.36,0.66]}, \frac{\left(u_{6}, 1\right)}{[0.80,0.90],[0.43,0.76]}\right)\right), \\
& \left(\left(k_{3}, k_{6}\right),\left(\frac{\left(u_{1}, 0\right)}{[0.40,0.73],[0.26,0.66]}, \frac{\left(u_{2}, 3\right)}{[0.66,0.86],[0.53,0.70]}, \frac{\left(u_{3}, 0\right)}{[0.50,0.66],[0.40,0.70]},\right.\right. \\
& \left.\left.\left.\frac{\left(u_{4}, 2\right)}{36],[0.40,0.56]}, \frac{\left(u_{5}, 1\right)}{[0.50,0.70],[0.46,0.73]}, \frac{\left(u_{6}, 1\right)}{[0.66,0.96],[0.30,0.70]}\right)\right)\right\} \text {. }
\end{aligned}
$$

Then we will evaluate the choice value $\mathbb{C}\left(k_{s^{\prime}}, l_{t^{\prime}}\right)\left(u_{i}\right) ; \forall u_{i} \in U,\left(k_{s^{\prime}}, l_{t^{\prime}}\right) \in(K \times L), s^{\prime}=$ 1,3 and $t^{\prime}=4,5,6$ by using step 3 of Algorithm 2:

\begin{tabular}{lclc}
\hline $\mathbb{C}\left(k_{s^{\prime}}, k_{t^{\prime}}\right)\left(u_{i}\right)$ & (Grading, Interval Value $)$ & $\mathbb{C}\left(k_{s^{\prime}}, k_{t^{\prime}}\right)\left(u_{i}\right)$ & (Grading, Interval Value $)$ \\
\hline $\mathbb{C}\left(k_{1}, k_{4}\right)\left(u_{1}\right)$ & $(1,[0.73,1.40])$ & $\mathbb{C}\left(k_{3}, k_{4}\right)\left(u_{1}\right)$ & $(0,[0.73,1.46])$ \\
\hline $\mathbb{C}\left(k_{1}, k_{4}\right)\left(u_{2}\right)$ & $(1,[0.96,1.63])$ & $\mathbb{C}\left(k_{3}, k_{4}\right)\left(u_{2}\right)$ & $(1,[0.93,1.66])$ \\
\hline $\mathbb{C}\left(k_{1}, k_{4}\right)\left(u_{3}\right)$ & $(1,[0.70,1.20])$ & $\mathbb{C}\left(k_{3}, k_{4}\right)\left(u_{3}\right)$ & $(2,[0.86,1.06])$ \\
\hline $\mathbb{C}\left(k_{1}, k_{4}\right)\left(u_{4}\right)$ & $(1,[0.96,1.43])$ & $\mathbb{C}\left(k_{3}, k_{4}\right)\left(u_{4}\right)$ & $(1,[0.63,1.33])$ \\
\hline $\mathbb{C}\left(k_{1}, k_{4}\right)\left(u_{5}\right)$ & $(0,[1.23,1.76])$ & $\mathbb{C}\left(k_{3}, k_{4}\right)\left(u_{5}\right)$ & $(1,[1.03,1.60])$ \\
\hline $\mathbb{C}\left(k_{1}, k_{4}\right)\left(u_{6}\right)$ & $(0,[0.80,1.60])$ & $\mathbb{C}\left(k_{3}, k_{4}\right)\left(u_{6}\right)$ & $(0,[0.86,1.73])$ \\
\hline $\mathbb{C}\left(k_{1}, k_{5}\right)\left(u_{1}\right)$ & $(0,[0.46,1.50])$ & $\mathbb{C}\left(k_{3}, k_{5}\right)\left(u_{1}\right)$ & $(0,[0.63,1.53])$ \\
\hline $\mathbb{C}\left(k_{1}, k_{5}\right)\left(u_{2}\right)$ & $(2,[1.26,1.70])$ & $\mathbb{C}\left(k_{3}, k_{5}\right)\left(u_{2}\right)$ & $(2,[1.26,1.73])$ \\
\hline $\mathbb{C}\left(k_{1}, k_{5}\right)\left(u_{3}\right)$ & $(1,[0.56,1.36])$ & $\mathbb{C}\left(k_{3}, k_{5}\right)\left(u_{3}\right)$ & $(2,[0.73,1.23])$ \\
\hline $\mathbb{C}\left(k_{1}, k_{5}\right)\left(u_{4}\right)$ & $(2,[1.13,1.50])$ & $\mathbb{C}\left(k_{3}, k_{5}\right)\left(u_{4}\right)$ & $(2,[0.93,1.40])$ \\
\hline $\mathbb{C}\left(k_{1}, k_{5}\right)\left(u_{5}\right)$ & $(0,[1.20,1.60])$ & $\mathbb{C}\left(k_{3}, k_{5}\right)\left(u_{5}\right)$ & $(1,[0.93,1.43])$ \\
\hline $\mathbb{C}\left(k_{1}, k_{5}\right)\left(u_{6}\right)$ & $(3,[1.13,1.50])$ & $\mathbb{C}\left(k_{3}, k_{5}\right)\left(u_{6}\right)$ & $(1,[1.23,1.66])$ \\
\hline $\mathbb{C}\left(k_{1}, k_{6}\right)\left(u_{1}\right)$ & $(1,[0.53,1.33])$ & $\mathbb{C}\left(k_{3}, k_{6}\right)\left(u_{1}\right)$ & $(0,[0.66,1.40])$ \\
\hline $\mathbb{C}\left(k_{1}, k_{6}\right)\left(u_{2}\right)$ & $(3,[1.06,1.53])$ & $\mathbb{C}\left(k_{3}, k_{6}\right)\left(u_{2}\right)$ & $(3,[1.20,1.56])$ \\
\hline $\mathbb{C}\left(k_{1}, k_{6}\right)\left(u_{3}\right)$ & $(0,[0.80,1.43])$ & $\mathbb{C}\left(k_{3}, k_{6}\right)\left(u_{3}\right)$ & $(0,[0.90,1.36])$ \\
\hline
\end{tabular}




\begin{tabular}{lccc}
\hline $\mathbb{C}\left(\boldsymbol{k}_{\boldsymbol{s}^{\prime}}, \boldsymbol{k}_{\boldsymbol{t}^{\prime}}\right)\left(u_{i}\right)$ & (Grading, Interval Value $)$ & $\mathbb{C}\left(\boldsymbol{k}_{\boldsymbol{s}^{\prime}}, \boldsymbol{k}_{\boldsymbol{t}^{\prime}}\right)\left(\boldsymbol{u}_{\boldsymbol{i}}\right)$ & (Grading, Interval Value) \\
\hline $\mathbb{C}\left(k_{1}, k_{6}\right)\left(u_{4}\right)$ & $(2,[0.90,1.16])$ & $\mathbb{C}\left(k_{3}, k_{6}\right)\left(u_{4}\right)$ & $(2,[0.56,0.93])$ \\
\hline $\mathbb{C}\left(k_{1}, k_{6}\right)\left(u_{5}\right)$ & $(0,[1.20,1.63])$ & $\mathbb{C}\left(k_{3}, k_{6}\right)\left(u_{5}\right)$ & $(1,[0.96,1.43])$ \\
\hline $\mathbb{C}\left(k_{1}, k_{6}\right)\left(u_{6}\right)$ & $(3,[0.86,1.53])$ & $\mathbb{C}\left(k_{3}, k_{6}\right)\left(u_{6}\right)$ & $(1,[0.96,1.66])$ \\
\hline
\end{tabular}

Now we will evaluate the Score $\mathbb{S}\left(k_{s^{\prime}}, l_{t^{\prime}}\right)\left(u_{i}\right) ; \forall u_{i} \in U,\left(k_{s^{\prime}}, l_{t^{\prime}}\right) \in(K \times L), \quad s^{\prime}=$ 1,3 and $t^{\prime}=4,5,6$ by using step 4 of Algorithm 2 .

\begin{tabular}{lccc}
\hline $\mathbb{S}\left(k_{s^{\prime}}, k_{t^{\prime}}\right)\left(u_{i}\right)$ & The Score & $\mathbb{S}\left(k_{s^{\prime}}, k_{t^{\prime}}\right)\left(u_{i}\right)$ & The Score \\
\hline $\mathbb{S}\left(k_{1}, k_{4}\right)\left(u_{1}\right)$ & -9.72 & $\mathbb{S}\left(k_{3}, k_{4}\right)\left(u_{1}\right)$ & -3.7 \\
\hline $\mathbb{S}\left(k_{1}, k_{4}\right)\left(u_{2}\right)$ & 6.84 & $\mathbb{S}\left(k_{3}, k_{4}\right)\left(u_{2}\right)$ & 11.62 \\
\hline $\mathbb{S}\left(k_{1}, k_{4}\right)\left(u_{3}\right)$ & -18 & $\mathbb{S}\left(k_{3}, k_{4}\right)\left(u_{3}\right)$ & -28.32 \\
\hline $\mathbb{S}\left(k_{1}, k_{4}\right)\left(u_{4}\right)$ & -0.36 & $\mathbb{S}\left(k_{3}, k_{4}\right)\left(u_{4}\right)$ & -14.84 \\
\hline $\mathbb{S}\left(k_{1}, k_{4}\right)\left(u_{5}\right)$ & 14.16 & $\mathbb{S}\left(k_{3}, k_{4}\right)\left(u_{5}\right)$ & 13.3 \\
\hline $\mathbb{S}\left(k_{1}, k_{4}\right)\left(u_{6}\right)$ & 0 & $\mathbb{S}\left(k_{3}, k_{4}\right)\left(u_{6}\right)$ & 8.3 \\
\hline $\mathbb{S}\left(k_{1}, k_{5}\right)\left(u_{1}\right)$ & -25.12 & $\mathbb{S}\left(k_{3}, k_{5}\right)\left(u_{1}\right)$ & -13.84 \\
\hline $\mathbb{S}\left(k_{1}, k_{5}\right)\left(u_{2}\right)$ & 37.18 & $\mathbb{S}\left(k_{3}, k_{5}\right)\left(u_{2}\right)$ & 39 \\
\hline $\mathbb{S}\left(k_{1}, k_{5}\right)\left(u_{3}\right)$ & -33.8 & $\mathbb{S}\left(k_{3}, k_{5}\right)\left(u_{3}\right)$ & -35.16 \\
\hline $\mathbb{S}\left(k_{1}, k_{5}\right)\left(u_{4}\right)$ & $\left.11.44, k_{5}\right)\left(u_{4}\right)$ & -4.77 \\
\hline $\mathbb{S}\left(k_{1}, k_{5}\right)\left(u_{5}\right)$ & 15.2 & $\mathbb{S}\left(k_{3}, k_{5}\right)\left(u_{5}\right)$ & 23.85 \\
\hline $\mathbb{S}\left(k_{1}, k_{5}\right)\left(u_{6}\right)$ & 15.84 & $\mathbb{S}\left(k_{3}, k_{5}\right)\left(u_{6}, k_{6}\right)\left(u_{1}\right)$ & -8.54 \\
\hline $\mathbb{S}\left(k_{1}, k_{6}\right)\left(u_{1}\right)$ & -30.8 & $\mathbb{S}\left(k_{3}, k_{6}\right)\left(u_{2}\right)$ & 53.64 \\
\hline $\mathbb{S}\left(k_{1}, k_{6}\right)\left(u_{2}\right)$ & $\mathbb{S}\left(k_{3}, k_{6}\right)\left(u_{3}\right)$ & -0.14 \\
\hline $\mathbb{S}\left(k_{1}, k_{6}\right)\left(u_{3}\right)$ & 28.44 & $\mathbb{S}\left(k_{3}, k_{6}\right)\left(u_{4}\right)$ & -60.32 \\
\hline $\mathbb{S}\left(k_{1}, k_{6}\right)\left(u_{4}\right)$ & -5.22 & $\mathbb{S}\left(k_{3}, k_{6}\right)\left(u_{5}\right)$ & 6.84 \\
\hline $\mathbb{S}\left(k_{1}, k_{6}\right)\left(u_{5}\right)$ & -22.4 & $\mathbb{S}\left(k_{3}, k_{6}\right)\left(u_{6}\right)$ & 19.26 \\
\hline $\mathbb{S}\left(k_{1}, k_{6}\right)\left(u_{6}\right)$ & 27.18 & & \\
\hline & 6.84 & &
\end{tabular}

By using step 5 of Algorithm 2 the weighted values for each $\mathbb{S}\left(u_{l}\right)$ is:

$$
\begin{gathered}
\mathbb{S}\left(u_{1}\right)=-91.72, \mathbb{S}\left(u_{2}\right)=1776.72, \mathbb{S}\left(u_{3}\right)=-120.64 \\
\mathbb{S}\left(u_{4}\right)=-95, \mathbb{S}\left(u_{5}\right)=71.91, \mathbb{S}\left(u_{6}\right)=74.09
\end{gathered}
$$

Here the optimal decision by using step 6 of Algorithm 2 is:

$$
\mathfrak{X}=\arg \max \left\{\mathbb{S}\left(u_{1}\right), \mathbb{S}\left(u_{2}\right), \mathbb{S}\left(u_{3}\right), \mathbb{S}\left(u_{4}\right), \mathbb{S}\left(u_{5}\right), \mathbb{S}\left(u_{6}\right)\right\} .
$$

Thus, $u_{2}$ is the best choice. Hence Mr. $\mathcal{H}$ will buy the $u_{2}$ smartphone.

In Figure 5, we observe that the following relations between the score values of Examples 11 and 12 by means of Algorithms 1 and 2, respectively.

$$
\begin{aligned}
& \mathbb{S}\left(u_{3}\right) \preceq \mathbb{S}\left(u_{4}\right) \preceq \mathbb{S}\left(u_{1}\right) \preceq \mathbb{S}\left(u_{6}\right) \preceq \mathbb{S}\left(u_{2}\right) \preceq \mathbb{S}\left(u_{5}\right), \\
& \mathbb{S}\left(u_{3}\right) \preceq \mathbb{S}\left(u_{4}\right) \preceq \mathbb{S}\left(u_{1}\right) \preceq \mathbb{S}\left(u_{5}\right) \preceq \mathbb{S}\left(u_{6}\right) \preceq \mathbb{S}\left(u_{2}\right) .
\end{aligned}
$$




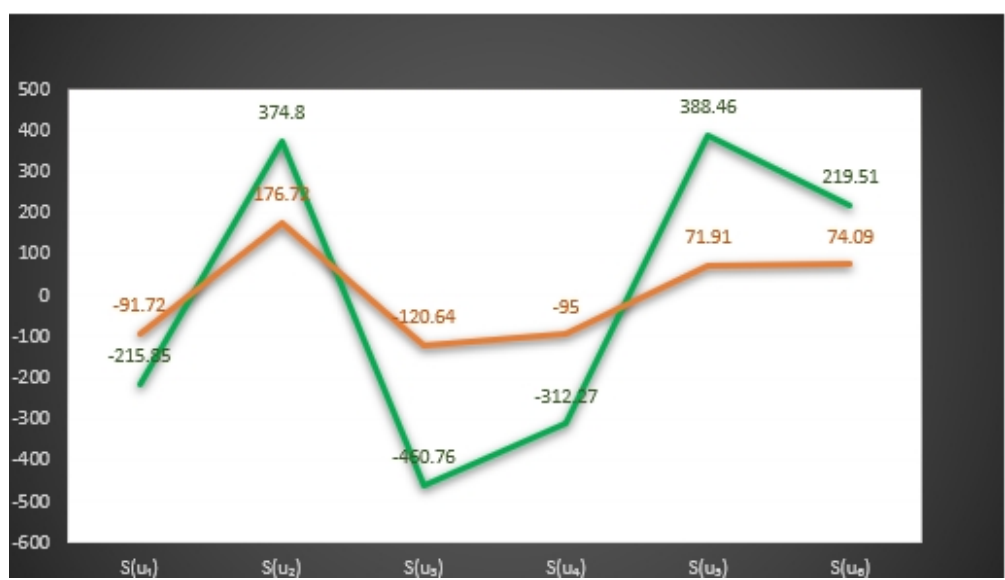

Figure 5. Comparison of score values of Examples 11 and 12 by means of Algorithms 1 and 2.

Example 13. Mr. X wants to select a personal secretary with remarkable qualities, and there are three persons under consideration. Let $U=\left\{u_{1}, u_{2}, u_{3}, u_{4}, u_{5}, u_{6}\right\}$ be the set of persons. Let $E=$ $\left\{k_{1}=\right.$ communication and planning skills, $k_{2}=$ multitasking skills, $k_{3}=$ time management, $k_{4}=$ judgement ability,$k_{5}=$ hardworking, $k_{6}=$ confident, $k_{7}=$ technology skills $\}$ be the set of parameters (remarkable qualities) for the selection of personal secretary and $K, L \subseteq E$ such that $K=\left\{k_{1}, k_{3}, k_{5}\right\}$ and $L=\left\{k_{4}, k_{5}, k_{7}\right\}$. Assume that $M r$. X wants to select one such person depending on two parameters only. Suppose there are two observations $\left(B_{b}, K, 5\right)$ and $\left(Y_{y}, L, 8\right)$ by two experts as follows:

$$
\begin{aligned}
& \left(B_{b}, K, 5\right)=\left\{\left(k_{1},\left(\frac{\left(u_{1}, 1\right)}{[0.5,0.8],[0.3,0.5]}, \frac{\left(u_{2}, 3\right)}{[0.2,0.9],[0.3,0.7]}, \frac{\left(u_{3}, 0\right)}{[0.1,0.3],[0.1,0.4]},\right.\right.\right. \\
& \begin{array}{lll}
\left(u_{4}, 2\right) & \left(u_{5}, 4\right) & \left(u_{6}, 3\right)
\end{array} \\
& \overline{[0.1,0.3],[0.5,0.6]}, \overline{[0.4,0.7],[0.3,0.9]}, \overline{[0.6,0.7],[0.3,0.6]})), \\
& \left(k_{3},\left(\frac{\left(u_{1}, 0\right)}{[0.4,0.9],[0.3,0.6]}, \frac{\left(u_{2}, 4\right)}{[0.4,0.7],[0.5,0.9]}, \frac{\left(u_{3}, 1\right)}{[0.8,0.9],[0.4,0.9]},\right.\right. \\
& \left.\left.\frac{\left(u_{4}, 3\right)}{[0.3,0.5],[0.4,0.6]}, \frac{\left(u_{5}, 2\right)}{[0.8,0.9],[0.3,0.7]}, \frac{\left(u_{6}, 4\right)}{[0.2,0.4],[0.3,0.5]}\right)\right), \\
& \left(k_{5},\left(\frac{\left(u_{1}, 4\right)}{[0.3,0.7],[0.2,0.5]}, \frac{\left(u_{2}, 3\right)}{[0.3,0.8],[0.2,0.9]}, \frac{\left(u_{3}, 2\right)}{[0.7,0.9],[0.5,0.8]},\right.\right. \\
& \left.\left.\left.\frac{\left(u_{4}, 3\right)}{[0.7,0.8],[0.5,0.6]}, \frac{\left(u_{5}, 0\right)}{[0.1,0.7],[0.3,0.4]}, \frac{\left(u_{6}, 1\right)}{[0.7,1.0],[0.5,0.8]}\right)\right)\right\} \text {. } \\
& \left(Y_{y}, L, 8\right)=\left\{\left(k_{4},\left(\frac{\left(u_{1}, 5\right)}{[0.1,0.7],[0.3,0.8]}, \frac{\left(u_{2}, 6\right)}{[0.5,0.7],[0.5,0.7]}, \frac{\left(u_{3}, 3\right)}{[0.3,0.4],[0.5,0.6]},\right.\right.\right. \\
& \left.\left.\frac{\left(u_{4}, 7\right)}{[0.1,0.6],[0.2,0.4]}, \frac{\left(u_{5}, 4\right)}{[0.5,0.6],[0.4,1.0]}, \frac{\left(u_{6}, 2\right)}{[0.3,0.6],[0.2,0.8]}\right)\right), \\
& \left(k_{5},\left(\frac{\left(u_{1}, 2\right)}{[0.3,0.5],[0.6,0.7]}, \frac{\left(u_{2}, 1\right)}{[0.3,0.9],[0.3,0.6]}, \frac{\left(u_{3}, 0\right)}{[0.2,0.3],[0.3,0.5]},\right.\right. \\
& \left.\left.\frac{\left(u_{4}, 5\right)}{[0.5,0.8],[0.6,0.7]}, \frac{\left(u_{5}, 4\right)}{[0.6,0.9],[0.7,0.8]}, \frac{\left(u_{6}, 3\right)}{[0.3,0.5],[0.2,0.6]}\right)\right), \\
& \left(k_{7},\left(\frac{\left(u_{1}, 7\right)}{[0.8,0.9],[0.4,0.5]}, \frac{\left(u_{2}, 4\right)}{[0.6,0.8],[0.4,0.7]}, \frac{\left(u_{3}, 5\right)}{[0.1,0.9],[0.1,0.8]},\right.\right. \\
& \left.\left.\left.\frac{\left(u_{4}, 3\right)}{[0.1,0.5],[0.2,0.7]}, \frac{\left(u_{5}, 2\right)}{[0.2,0.3],[0.4,0.5]}, \frac{\left(u_{6}, 6\right)}{[0.4,0.7],[0.5,0.6]}\right)\right)\right\} \text {. }
\end{aligned}
$$


Firstly, we will evaluate the soft max-AND operation $\left(G_{g}, K \times L, 8\right)=\left(B_{b}, K, 5\right) \widehat{\wedge}\left(Y_{y}, L, 8\right)$ by using step 2 of Algorithm 1:

$$
\begin{aligned}
& \left(G_{g}, K \times L, 8\right)=\left\{\left(\left(k_{1}, k_{4}\right),\left(\frac{\left(u_{1}, 5\right)}{[0.36,0.76],[0.30,0.70]}, \frac{\left(u_{2}, 6\right)}{[0.40,0.83],[0.43,0.70]}, \frac{\left(u_{3}, 3\right)}{[0.23,0.36],[0.36,0.53]},\right.\right.\right. \\
& \left.\left.\frac{\left(u_{4}, 7\right)}{[0.10,0.50],[0.40,0.53]}, \frac{\left(u_{5}, 4\right)}{[0.46,0.66],[0.36,0.96]}, \frac{\left(u_{6}, 3\right)}{[0.50,0.66],[0.26,0.73]}\right)\right) \text {, } \\
& \left(\left(k_{1}, k_{5}\right),\left(\frac{\left(u_{1}, 2\right)}{[0.43,0.70],[0.50,0.63]}, \frac{\left(u_{2}, 3\right)}{[0.26,0.90],[0.30,0.66]}, \frac{\left(u_{3}, 0\right)}{[0.16,0.30],[0.23,0.46]},\right.\right. \\
& \left.\left.\frac{\left(u_{4}, 5\right)}{[0.36,0.63],[0.56,0.66]}, \frac{\left(u_{5}, 4\right)}{[0.53,0.83],[0.56,0.86]}, \frac{\left(u_{6}, 3\right)}{[0.50,0.63],[0.26,0.60]}\right)\right), \\
& \left(\left(k_{1}, k_{7}\right),\left(\frac{\left(u_{1}, 7\right)}{[0.70,0.86],[0.36,0.50]}, \frac{\left(u_{2}, 4\right)}{[0.46,0.86],[0.36,0.70]}, \frac{\left(u_{3}, 5\right)}{[0.10,0.70],[0.10,0.66]},\right.\right. \\
& \left.\left.\frac{\left(u_{4}, 3\right)}{0.43],[0.40,0.66]}, \frac{\left(u_{5}, 4\right)}{[0.33,0.56],[0.36,0.76]}, \frac{\left(u_{6}, 6\right)}{[0.53,0.70],[0.43,0.60]}\right)\right) \text {, } \\
& \left(\left(k_{3}, k_{4}\right),\left(\frac{\left(u_{1}, 5\right)}{[0.30,0.83],[0.30,0.73]}, \frac{\left(u_{2}, 6\right)}{[0.46,0.70],[0.50,0.83]}, \frac{\left(u_{3}, 3\right)}{[0.63,0.73],[0.46,0.80]},\right.\right. \\
& \left.\left.\frac{\left(u_{4}, 7\right)}{0.56],[0.33,0.53]}, \frac{\left(u_{5}, 4\right)}{[0.70,0.80],[0.36,0.90]}, \frac{\left(u_{6}, 4\right)}{[0.26,0.53],[0.26,0.70]}\right)\right) \text {, } \\
& \left(\left(k_{3}, k_{5}\right),\left(\frac{\left(u_{1}, 2\right)}{[0.36,0.76],[0.50,0.66]}, \frac{\left(u_{2}, 4\right)}{[0.36,0.83],[0.43,0.80]}, \frac{\left(u_{3}, 1\right)}{[0.60,0.70],[0.36,0.76]},\right.\right. \\
& \left.\left.\frac{\left(u_{4}, 5\right)}{[0.43,0.70],[0.53,0.66]}, \frac{\left(u_{5}, 4\right)}{[0.73,0.90],[0.56,0.76]}, \frac{\left(u_{6}, 4\right)}{[0.26,0.46],[0.26,0.56]}\right)\right), \\
& \left(\left(k_{3}, k_{7}\right),\left(\frac{\left(u_{1}, 7\right)}{[0.66,0.90],[0.36,0.56]}, \frac{\left(u_{2}, 4\right)}{[0.53,0.76],[0.46,0.83]}, \frac{\left(u_{3}, 5\right)}{[0.56,0.90],[0.30,0.86]},\right.\right. \\
& \left.\left.\frac{\left(u_{4}, 3\right)}{[0.23,0.50],[0.33,0.66]}, \frac{\left(u_{5}, 2\right)}{[0.60,0.70],[0.36,0.63]}, \frac{\left(u_{6}, 6\right)}{[0.33,0.60],[0.43,0.56]}\right)\right), \\
& \left(\left(k_{5}, k_{4}\right),\left(\frac{\left(u_{1}, 5\right)}{[0.23,0.70],[0.26,0.70]}, \frac{\left(u_{2}, 6\right)}{[0.43,0.76],[0.40,0.83]}, \frac{\left(u_{3}, 3\right)}{[0.56,0.73],[0.50,0.73]},\right.\right. \\
& \left.\left.\frac{\left(u_{4}, 7\right)}{0.73],[0.40,0.53]}, \frac{\left(u_{5}, 4\right)}{[0.36,0.66],[0.36,0.80]}, \frac{\left(u_{6}, 2\right)}{[0.56,0.86],[0.40,0.80]}\right)\right), \\
& \left(\left(k_{5}, k_{5}\right),\left(\frac{\left(u_{1}, 4\right)}{[0.30,0.63],[0.46,0.63]}, \frac{\left(u_{2}, 3\right)}{[0.30,0.86],[0.26,0.80]}, \frac{\left(u_{3}, 2\right)}{[0.53,0.70],[0.43,0.70]},\right.\right. \\
& \left.\left.\frac{\left(u_{4}, 5\right)}{0.80],[0.56,0.66]}, \frac{\left(u_{5}, 4\right)}{[0.43,0.83],[0.56,0.66]}, \frac{\left(u_{6}, 3\right)}{[0.56,0.83],[0.40,0.73]}\right)\right), \\
& \left(\left(k_{5}, k_{7}\right),\left(\frac{\left(u_{1}, 7\right)}{[0.63,0.83],[0.33,0.50]}, \frac{\left(u_{2}, 4\right)}{[0.50,0.80],[0.33,0.83]}, \frac{\left(u_{3}, 5\right)}{[0.50,0.90],[0.36,0.80]},\right.\right. \\
& \left.\left.\left.\frac{\left(u_{4}, 3\right)}{[0.50,0.70],[0.40,0.66]}, \frac{\left(u_{5}, 2\right)}{[0.16,0.56],[0.36,0.46]}, \frac{\left(u_{6}, 6\right)}{[0.60,0.90],[0.50,0.73]}\right)\right)\right\} \text {. }
\end{aligned}
$$

Then we will evaluate the choice value $\mathbb{C}\left(k_{s^{\prime}}, l_{t^{\prime}}\right)\left(u_{i}\right) ; \forall u_{i} \in U,\left(k_{s^{\prime}}, l_{t^{\prime}}\right) \in(K \times L), s^{\prime}=$ $1,3,5$ and $t^{\prime}=4,5,7$ by using step 3 of Algorithm 1:

\begin{tabular}{lccc}
\hline $\mathbb{C}\left(\boldsymbol{k}_{\boldsymbol{s}^{\prime}}, \boldsymbol{k}_{\boldsymbol{t}^{\prime}}\right)\left(u_{i}\right)$ & $($ Grading, Interval Value $)$ & $\mathbb{C}\left(\boldsymbol{k}_{\boldsymbol{s}^{\prime}}, \boldsymbol{k}_{\boldsymbol{t}^{\prime}}\right)\left(u_{\boldsymbol{i}}\right)$ & (Grading, Interval Value $)$ \\
\hline $\mathbb{C}\left(k_{1}, k_{4}\right)\left(u_{1}\right)$ & $(5,[0.53,1.33])$ & $\mathbb{C}\left(k_{3}, k_{5}\right)\left(u_{4}\right)$ & $(5,[0.83,1.23])$ \\
\hline $\mathbb{C}\left(k_{1}, k_{4}\right)\left(u_{2}\right)$ & $(6,[0.66,1.46])$ & $\mathbb{C}\left(k_{3}, k_{5}\right)\left(u_{5}\right)$ & $(4,[1.10,1.63])$ \\
\hline $\mathbb{C}\left(k_{1}, k_{4}\right)\left(u_{3}\right)$ & $(3,[0.40,0.80])$ & $\mathbb{C}\left(k_{3}, k_{5}\right)\left(u_{6}\right)$ & $(4,[0.46,0.96])$ \\
\hline $\mathbb{C}\left(k_{1}, k_{4}\right)\left(u_{4}\right)$ & $(7,[0.40,0.86])$ & $\mathbb{C}\left(k_{3}, k_{7}\right)\left(u_{1}\right)$ & $(7,[0.86,1.43])$ \\
\hline $\mathbb{C}\left(k_{1}, k_{4}\right)\left(u_{5}\right)$ & $(4,[0.76,1.56])$ & $\mathbb{C}\left(k_{3}, k_{7}\right)\left(u_{2}\right)$ & $(4,[0.90,1.50])$ \\
\hline $\mathbb{C}\left(k_{1}, k_{4}\right)\left(u_{6}\right)$ & $(3,[0.63,1.30])$ & $\mathbb{C}\left(k_{3}, k_{7}\right)\left(u_{3}\right)$ & $(5,[0.53,1.73])$ \\
\hline $\mathbb{C}\left(k_{1}, k_{5}\right)\left(u_{1}\right)$ & $(2,[0.76,1.16])$ & $\mathbb{C}\left(k_{3}, k_{7}\right)\left(u_{4}\right)$ & $(3,[0.43,1.13])$ \\
\hline
\end{tabular}




\begin{tabular}{|c|c|c|c|}
\hline $\mathbb{C}\left(k_{s^{\prime}}, k_{t^{\prime}}\right)\left(u_{i}\right)$ & (Grading, Interval Value) & $\mathbb{C}\left(k_{s^{\prime}}, k_{t^{\prime}}\right)\left(u_{i}\right)$ & (Grading, Interval Value) \\
\hline $\mathbb{C}\left(k_{1}, k_{5}\right)\left(u_{2}\right)$ & $(3,[0.53,1.53])$ & $\mathbb{C}\left(k_{3}, k_{7}\right)\left(u_{5}\right)$ & $(2,[0.73,1.06])$ \\
\hline $\mathbb{C}\left(k_{1}, k_{5}\right)\left(u_{3}\right)$ & $(0,[0.30,1.73])$ & $\mathbb{C}\left(k_{3}, k_{7}\right)\left(u_{6}\right)$ & $(6,[0.63,1.03])$ \\
\hline $\mathbb{C}\left(k_{1}, k_{5}\right)\left(u_{4}\right)$ & $(5,[0.76,1.10])$ & $\mathbb{C}\left(k_{5}, k_{4}\right)\left(u_{1}\right)$ & $(5,[0.40,1.30])$ \\
\hline $\mathbb{C}\left(k_{1}, k_{5}\right)\left(u_{5}\right)$ & $(4,[0.90,1.60])$ & $\mathbb{C}\left(k_{5}, k_{4}\right)\left(u_{2}\right)$ & $(6,[0.67,1.50])$ \\
\hline $\mathbb{C}\left(k_{1}, k_{5}\right)\left(u_{6}\right)$ & $(3,[0.63,1.16])$ & $\mathbb{C}\left(k_{5}, k_{4}\right)\left(u_{3}\right)$ & $(3,[0.93,1.23])$ \\
\hline $\mathbb{C}\left(k_{1}, k_{7}\right)\left(u_{1}\right)$ & $(7,[0.93,1.33])$ & $\mathbb{C}\left(k_{5}, k_{4}\right)\left(u_{4}\right)$ & $(7,[0.60,1.13])$ \\
\hline $\mathbb{C}\left(k_{1}, k_{7}\right)\left(u_{2}\right)$ & $(4,[0.66,1.53])$ & $\mathbb{C}\left(k_{5}, k_{4}\right)\left(u_{5}\right)$ & $(4,[0.56,1.23])$ \\
\hline $\mathbb{C}\left(k_{1}, k_{7}\right)\left(u_{3}\right)$ & $(5,[0.20,1.03])$ & $\mathbb{C}\left(k_{5}, k_{4}\right)\left(u_{6}\right)$ & $(2,[0.73,1.53])$ \\
\hline $\mathbb{C}\left(k_{1}, k_{7}\right)\left(u_{4}\right)$ & $(3,[0.40,1.00])$ & $\mathbb{C}\left(k_{5}, k_{5}\right)\left(u_{1}\right)$ & $(4,[0.63,1.13])$ \\
\hline $\mathbb{C}\left(k_{1}, k_{7}\right)\left(u_{5}\right)$ & $(4,[0.60,1.06])$ & $\mathbb{C}\left(k_{5}, k_{5}\right)\left(u_{2}\right)$ & $(3,[0.53,1.53])$ \\
\hline $\mathbb{C}\left(k_{1}, k_{7}\right)\left(u_{6}\right)$ & $(6,[0.83,1.30])$ & $\mathbb{C}\left(k_{5}, k_{5}\right)\left(u_{3}\right)$ & $(2,[0.73,1.10])$ \\
\hline $\mathbb{C}\left(k_{3}, k_{4}\right)\left(u_{1}\right)$ & $(5,[0.50,1.43])$ & $\mathbb{C}\left(k_{5}, k_{5}\right)\left(u_{4}\right)$ & $(5,[1.10,1.43])$ \\
\hline $\mathbb{C}\left(k_{3}, k_{4}\right)\left(u_{2}\right)$ & $(6,[0.93,1.46])$ & $\mathbb{C}\left(k_{5}, k_{5}\right)\left(u_{5}\right)$ & $(4,[0.70,1.30])$ \\
\hline $\mathbb{C}\left(k_{3}, k_{4}\right)\left(u_{3}\right)$ & $(3,[1.90,1.26])$ & $\mathbb{C}\left(k_{5}, k_{5}\right)\left(u_{6}\right)$ & $(3,[0.73,1.33])$ \\
\hline $\mathbb{C}\left(k_{3}, k_{4}\right)\left(u_{4}\right)$ & $(7,[0.43,1.00])$ & $\mathbb{C}\left(k_{5}, k_{7}\right)\left(u_{1}\right)$ & $(7,[0.73,1.26])$ \\
\hline $\mathbb{C}\left(k_{3}, k_{4}\right)\left(u_{5}\right)$ & $(4,[0.93,1.50])$ & $\mathbb{C}\left(k_{5}, k_{7}\right)\left(u_{2}\right)$ & $(4,[0.66,1.56])$ \\
\hline $\mathbb{C}\left(k_{3}, k_{4}\right)\left(u_{6}\right)$ & $(4,[0.46,1.06])$ & $\mathbb{C}\left(k_{5}, k_{7}\right)\left(u_{3}\right)$ & $(5,[0.53,1.70])$ \\
\hline $\mathbb{C}\left(k_{3}, k_{5}\right)\left(u_{1}\right)$ & $(2,[0.73,1.26])$ & $\mathbb{C}\left(k_{5}, k_{7}\right)\left(u_{4}\right)$ & $(3,[0.60,1.23])$ \\
\hline $\mathbb{C}\left(k_{3}, k_{5}\right)\left(u_{2}\right)$ & $(4,[0.70,1.46])$ & $\mathbb{C}\left(k_{5}, k_{7}\right)\left(u_{5}\right)$ & $(2,[0.46,0.86])$ \\
\hline $\mathbb{C}\left(k_{3}, k_{5}\right)\left(u_{3}\right)$ & $(1,[0.73,1.13])$ & $\mathbb{C}\left(k_{5}, k_{7}\right)\left(u_{6}\right)$ & $(6,[1.00,1.46])$ \\
\hline
\end{tabular}

Now we will evaluate the Score $\mathbb{S}\left(k_{s^{\prime}}, l_{t^{\prime}}\right)\left(u_{i}\right) ; \forall u_{i} \in U,\left(k_{s^{\prime}}, l_{t^{\prime}}\right) \in(K \times L), \quad s^{\prime}=$ $1,3,5$ and $t^{\prime}=4,5,7$ by using step 4 of Algorithm 1 .

\begin{tabular}{lccc}
\hline $\mathbb{S}\left(k_{s^{\prime}}, k_{t^{\prime}}\right)\left(u_{i}\right)$ & The Score & $\mathbb{S}\left(k_{s^{\prime}}, k_{t^{\prime}}\right)\left(u_{i}\right)$ & The Score \\
\hline $\mathbb{S}\left(k_{1}, k_{4}\right)\left(u_{1}\right)$ & 15.51 & $\mathbb{S}\left(k_{3}, k_{5}\right)\left(u_{4}\right)$ & 4.2 \\
\hline $\mathbb{S}\left(k_{1}, k_{4}\right)\left(u_{2}\right)$ & 75.11 & $\mathbb{S}\left(k_{3}, k_{5}\right)\left(u_{5}\right)$ & 104 \\
\hline $\mathbb{S}\left(k_{1}, k_{4}\right)\left(u_{3}\right)$ & -97.72 & $\mathbb{S}\left(k_{3}, k_{5}\right)\left(u_{6}\right)$ & -92.5 \\
\hline $\mathbb{S}\left(k_{1}, k_{4}\right)\left(u_{4}\right)$ & -131.46 & $\mathbb{S}\left(k_{3}, k_{7}\right)\left(u_{1}\right)$ & 74.76 \\
\hline $\mathbb{S}\left(k_{1}, k_{4}\right)\left(u_{5}\right)$ & 96.9 & $\mathbb{S}\left(k_{3}, k_{7}\right)\left(u_{2}\right)$ & 73.2 \\
\hline $\mathbb{S}\left(k_{1}, k_{4}\right)\left(u_{6}\right)$ & 24.92 & $\mathbb{S}\left(k_{3}, k_{7}\right)\left(u_{3}\right)$ & 52.8 \\
\hline $\mathbb{S}\left(k_{1}, k_{5}\right)\left(u_{1}\right)$ & -12.16 & $\mathbb{S}\left(k_{3}, k_{7}\right)\left(u_{4}\right)$ & -72.8 \\
\hline $\mathbb{S}\left(k_{1}, k_{5}\right)\left(u_{2}\right)$ & 4.2 & $\mathbb{S}\left(k_{3}, k_{7}\right)\left(u_{5}\right)$ & -32.94 \\
\hline $\mathbb{S}\left(k_{1}, k_{5}\right)\left(u_{3}\right)$ & 0.34 & $\mathbb{S}\left(k_{3}, k_{7}\right)\left(u_{6}\right)$ & -74 \\
\hline $\mathbb{S}\left(k_{1}, k_{5}\right)\left(u_{4}\right)$ & -30 & $\mathbb{S}\left(k_{5}, k_{4}\right)\left(u_{1}\right)$ & -53.13 \\
\hline $\mathbb{S}\left(k_{1}, k_{5}\right)\left(u_{5}\right)$ & 71 & $\mathbb{S}\left(k_{5}, k_{4}\right)\left(u_{2}\right)$ & 44.77 \\
\hline $\mathbb{S}\left(k_{1}, k_{5}\right)\left(u_{6}\right)$ & -29.82 & $\mathbb{S}\left(k_{5}, k_{4}\right)\left(u_{3}\right)$ & 32.2 \\
\hline
\end{tabular}




\begin{tabular}{lccc}
\hline $\mathbb{S}\left(k_{s^{\prime}}, k_{t^{\prime}}\right)\left(u_{i}\right)$ & The Score & $\mathbb{S}\left(k_{s^{\prime}}, k_{t^{\prime}}\right)\left(u_{i}\right)$ & The Score \\
\hline $\mathbb{S}\left(k_{1}, k_{7}\right)\left(u_{1}\right)$ & 112.98 & $\mathbb{S}\left(k_{5}, k_{4}\right)\left(u_{4}\right)$ & -60.06 \\
\hline $\mathbb{S}\left(k_{1}, k_{7}\right)\left(u_{2}\right)$ & 68.1 & $\mathbb{S}\left(k_{5}, k_{4}\right)\left(u_{5}\right)$ & -32.1 \\
\hline $\mathbb{S}\left(k_{1}, k_{7}\right)\left(u_{3}\right)$ & -115.17 & $\mathbb{S}\left(k_{5}, k_{4}\right)\left(u_{6}\right)$ & 47.25 \\
\hline $\mathbb{S}\left(k_{1}, k_{7}\right)\left(u_{4}\right)$ & -71.63 & $\mathbb{S}\left(k_{5}, k_{5}\right)\left(u_{1}\right)$ & -42 \\
\hline $\mathbb{S}\left(k_{1}, k_{7}\right)\left(u_{5}\right)$ & -27.3 & $\mathbb{S}\left(k_{5}, k_{5}\right)\left(u_{2}\right)$ & 2.64 \\
\hline $\mathbb{S}\left(k_{1}, k_{7}\right)\left(u_{6}\right)$ & 70.67 & $\mathbb{S}\left(k_{5}, k_{5}\right)\left(u_{3}\right)$ & -26.46 \\
\hline $\mathbb{S}\left(k_{3}, k_{4}\right)\left(u_{1}\right)$ & -9.24 & $\mathbb{S}\left(k_{5}, k_{5}\right)\left(u_{4}\right)$ & 88.2 \\
\hline $\mathbb{S}\left(k_{3}, k_{4}\right)\left(u_{2}\right)$ & 91.76 & $\mathbb{S}\left(k_{5}, k_{5}\right)\left(u_{5}\right)$ & -6 \\
\hline $\mathbb{S}\left(k_{3}, k_{4}\right)\left(u_{3}\right)$ & 31.9 & $\mathbb{S}\left(k_{5}, k_{5}\right)\left(u_{6}\right)$ & 2.64 \\
\hline $\mathbb{S}\left(k_{3}, k_{4}\right)\left(u_{4}\right)$ & $\mathbb{S}\left(k_{5}, k_{7}\right)\left(u_{1}\right)$ & -4.62 \\
\hline $\mathbb{S}\left(k_{3}, k_{4}\right)\left(u_{5}\right)$ & $\mathbb{S}\left(k_{5}, k_{7}\right)\left(u_{2}\right)$ & 38.1 \\
\hline $\mathbb{S}\left(k_{3}, k_{4}\right)\left(u_{6}\right)$ & 81.6 & $\mathbb{S}\left(k_{5}, k_{7}\right)\left(u_{3}\right)$ & -29.96 \\
\hline $\mathbb{S}\left(k_{3}, k_{5}\right)\left(u_{1}\right)$ & -82.2 & $\mathbb{S}\left(k_{5}, k_{7}\right)\left(u_{4}\right)$ & -111.51 \\
\hline $\mathbb{S}\left(k_{3}, k_{5}\right)\left(u_{2}\right)$ & -5.88 & $\mathbb{S}\left(k_{5}, k_{7}\right)\left(u_{5}\right)$ & 100.27 \\
\hline $\mathbb{S}\left(k_{3}, k_{5}\right)\left(u_{3}\right)$ & 18.5 & $\mathbb{S}\left(k_{5}, k_{7}\right)\left(u_{6}\right)$ &
\end{tabular}

By using step 5 of Algorithm 1 the weighted values for each $\mathbb{S}\left(u_{l}\right)$ is:

$$
\begin{gathered}
\mathbb{S}\left(u_{1}\right)=76.22, \quad \mathbb{S}\left(u_{2}\right)=416.38, \mathbb{S}\left(u_{3}\right)=-99.42, \\
\mathbb{S}\left(u_{4}\right)=-441.27, \mathbb{S}\left(u_{5}\right)=143.65, \mathbb{S}\left(u_{6}\right)=-32.77 .
\end{gathered}
$$

Here the optimal decision by using step 6 of Algorithm 1 is:

$$
\mathfrak{X}=\arg \max \left\{\mathbb{S}\left(u_{1}\right), \mathbb{S}\left(u_{2}\right), \mathbb{S}\left(u_{3}\right), \mathbb{S}\left(u_{4}\right), \mathbb{S}\left(u_{5}\right), \mathbb{S}\left(u_{6}\right)\right\} .
$$

Thus, $u_{2}$ is the best choice.

Hence $M r$. X will select $u_{2}$ person as a personal secretary.

In Figures 6 and 7, we give the graphical behavior of score values of Examples 13 and 14 by means of Algorithms 1 and 2 respectively.

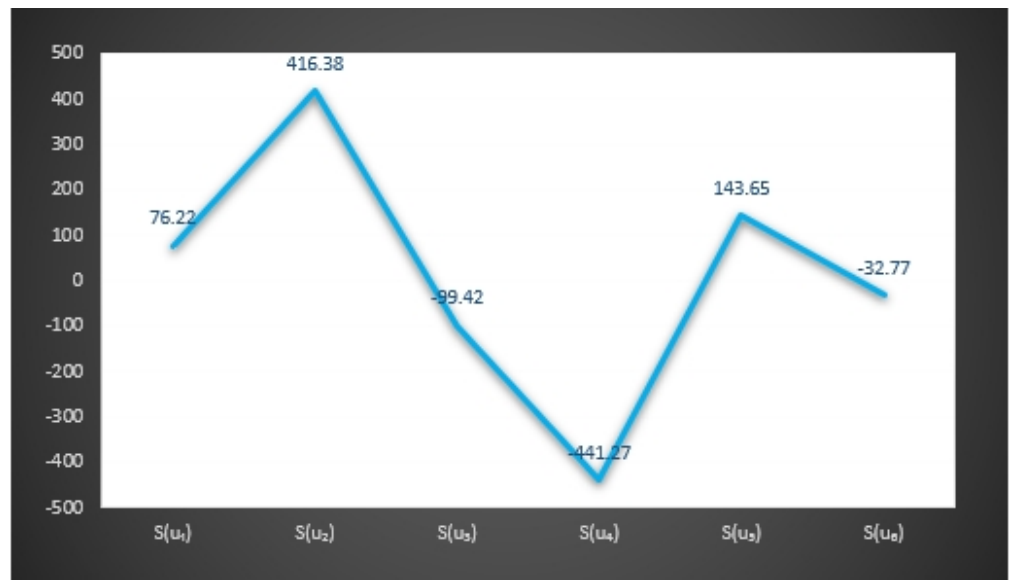

Figure 6. Graphical behavior of score values of Example 13 by means of Algorithm 1. 


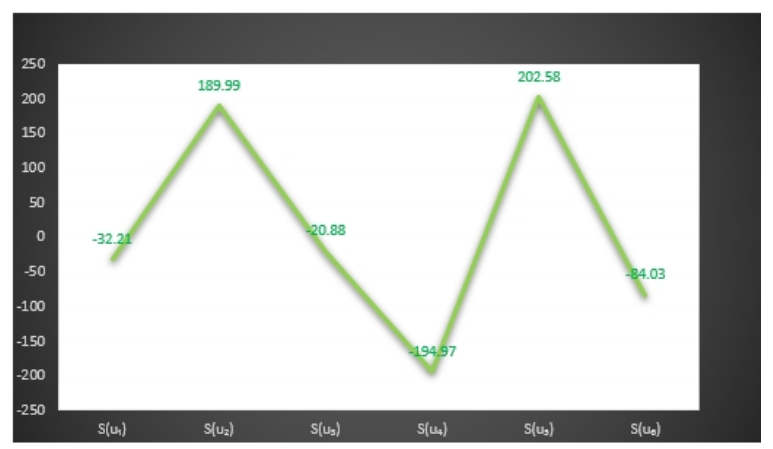

Figure 7. Graphical behavior of score values of Example 14 by means of Algorithm 2.

Example 14. Consider the Example 13 and assume that Mr. X wants to select one such person depending on one of two parameters only. Suppose there are two observations $\left(B_{b}, K, 5\right)$ and $\left(Y_{y}, L, 8\right)$ by two experts as follows:

Firstly, we will evaluate the soft min-OR operation $\left(Q_{q}, K \times L, 5\right)=\left(B_{b}, K, 5\right) \widehat{V}\left(Y_{y}, L, 8\right)$ by using step 2 of Algorithm 2:

$$
\begin{aligned}
& \left(Q_{q}, K \times L, 5\right)=\left\{\left(\left(k_{1}, k_{4}\right),\left(\frac{\left(u_{1}, 1\right)}{[0.36,0.76],[0.30,0.70]}, \frac{\left(u_{2}, 3\right)}{[0.40,0.83],[0.43,0.70]}, \frac{\left(u_{3}, 0\right)}{[0.23,0.36],[0.36,0.53]},\right.\right.\right. \\
& \left(u_{4}, 2\right) \quad\left(u_{5}, 4\right) \quad\left(u_{6}, 2\right) \\
& \overline{[0.10,0.50],[0.40,0.53]}, \overline{[0.46,0.66],[0.36,0.96]}, \overline{[0.50,0.66],[0.26,0.73]})), \\
& \left(\left(k_{1}, k_{5}\right),\left(\frac{\left(u_{1}, 1\right)}{[0.43,0.70],[0.50,0.63]}, \frac{\left(u_{2}, 1\right)}{[0.26,0.90],[0.30,0.66]}, \frac{\left(u_{3}, 0\right)}{[0.16,0.30],[0.23,0.46]},\right.\right. \\
& \left.\left.\frac{\left(u_{4}, 2\right)}{[0.36,0.63],[0.56,0.66]}, \frac{\left(u_{5}, 4\right)}{[0.53,0.83],[0.56,0.86]}, \frac{\left(u_{6}, 3\right)}{[0.50,0.63],[0.26,0.60]}\right)\right), \\
& \left(\left(k_{1}, k_{7}\right),\left(\frac{\left(u_{1}, 1\right)}{[0.70,0.86],[0.36,0.50]}, \frac{\left(u_{2}, 3\right)}{[0.46,0.86],[0.36,0.70]}, \frac{\left(u_{3}, 0\right)}{[0.10,0.70],[0.10,0.66]},\right.\right. \\
& \left.\left.\frac{\left(u_{4}, 2\right)}{[0.10,0.43],[0.40,0.66]}, \frac{\left(u_{5}, 2\right)}{[0.33,0.56],[0.36,0.76]}, \frac{\left(u_{6}, 3\right)}{[0.53,0.70],[0.43,0.60]}\right)\right), \\
& \left(\left(k_{3}, k_{4}\right),\left(\frac{\left(u_{1}, 0\right)}{[0.30,0.83],[0.30,0.73]}, \frac{\left(u_{2}, 4\right)}{[0.46,0.70],[0.50,0.83]}, \frac{\left(u_{3}, 1\right)}{[0.63,0.73],[0.46,0.80]},\right.\right. \\
& \left.\left.\frac{\left(u_{4}, 3\right)}{0.56],[0.33,0.53]}, \frac{\left(u_{5}, 2\right)}{[0.70,0.80],[0.36,0.90]}, \frac{\left(u_{6}, 2\right)}{[0.26,0.53],[0.26,0.70]}\right)\right), \\
& \left(\left(k_{3}, k_{5}\right),\left(\frac{\left(u_{1}, 0\right)}{[0.36,0.76],[0.50,0.66]}, \frac{\left(u_{2}, 1\right)}{[0.36,0.83],[0.43,0.80]}, \frac{\left(u_{3}, 0\right)}{[0.60,0.70],[0.36,0.76]},\right.\right. \\
& \left.\left.\frac{\left(u_{4}, 3\right)}{[0.43,0.70],[0.53,0.66]}, \frac{\left(u_{5}, 2\right)}{[0.73,0.90],[0.56,0.76]}, \frac{\left(u_{6}, 3\right)}{[0.26,0.46],[0.26,0.56]}\right)\right), \\
& \left(\left(k_{3}, k_{7}\right),\left(\frac{\left(u_{1}, 0\right)}{[0.66,0.90],[0.36,0.56]}, \frac{\left(u_{2}, 4\right)}{[0.53,0.76],[0.46,0.83]}, \frac{\left(u_{3}, 1\right)}{[0.56,0.90],[0.30,0.86]},\right.\right. \\
& \left.\left.\frac{\left(u_{4}, 3\right)}{[0.23,0.50],[0.33,0.66]}, \frac{\left(u_{5}, 2\right)}{[0.60,0.70],[0.36,0.63]}, \frac{\left(u_{6}, 4\right)}{[0.33,0.60],[0.43,0.56]}\right)\right), \\
& \left(\left(k_{5}, k_{4}\right),\left(\frac{\left(u_{1}, 4\right)}{[0.23,0.70],[0.26,0.70]}, \frac{\left(u_{2}, 3\right)}{[0.43,0.76],[0.40,0.83]}, \frac{\left(u_{3}, 2\right)}{[0.56,0.73],[0.50,0.73]},\right.\right. \\
& \left(u_{4}, 3\right) \quad\left(u_{5}, 0\right) \quad\left(u_{6}, 1\right) \\
& \overline{[0.50,0.73],[0.40,0.53]}, \overline{[0.36,0.66],[0.36,0.80]}, \overline{[0.56,0.86],[0.40,0.80]})), \\
& \left(\left(k_{5}, k_{5}\right),\left(\frac{\left(u_{1}, 2\right)}{[0.30,0.63],[0.46,0.63]}, \frac{\left(u_{2}, 1\right)}{[0.30,0.86],[0.26,0.80]}, \frac{\left(u_{3}, 0\right)}{[0.53,0.70],[0.43,0.70]},\right.\right. \\
& \left.\left.\frac{\left(u_{4}, 3\right)}{[0.63,0.80],[0.56,0.66]}, \frac{\left(u_{5}, 0\right)}{[0.43,0.83],[0.56,0.66]}, \frac{\left(u_{6}, 1\right)}{[0.56,0.83],[0.40,0.73]}\right)\right), \\
& \left(\left(k_{5}, k_{7}\right),\left(\frac{\left(u_{1}, 4\right)}{[0.63,0.83],[0.33,0.50]}, \frac{\left(u_{2}, 3\right)}{[0.50,0.80],[0.33,0.83]}, \frac{\left(u_{3}, 2\right)}{[0.50,0.90],[0.36,0.80]},\right.\right. \\
& \left.\left.\left.\frac{\left(u_{4}, 3\right)}{[0.50,0.70],[0.40,0.66]}, \frac{\left(u_{5}, 0\right)}{[0.16,0.56],[0.36,0.46]}, \frac{\left(u_{6}, 1\right)}{[0.60,0.90],[0.50,0.73]}\right)\right)\right\} \text {. }
\end{aligned}
$$


Then we will evaluate the choice value $\mathbb{C}\left(k_{s^{\prime}}, l_{t^{\prime}}\right)\left(u_{i}\right) ; \forall u_{i} \in U,\left(k_{s^{\prime}}, l_{t^{\prime}}\right) \in(K \times L), s^{\prime}=$ $1,3,5$ and $t^{\prime}=4,5,7$ by using step 3 of Algorithm 2:

\begin{tabular}{|c|c|c|c|}
\hline $\mathbb{C}\left(k_{s^{\prime}}, k_{t^{\prime}}\right)\left(u_{i}\right)$ & (Grading, Interval Value) & $\mathbb{C}\left(k_{s^{\prime}}, k_{t^{\prime}}\right)\left(u_{i}\right)$ & (Grading, Interval Value) \\
\hline $\mathbb{C}\left(k_{1}, k_{4}\right)\left(u_{1}\right)$ & $(1,[0.66,1.46])$ & $\mathbb{C}\left(k_{3}, k_{5}\right)\left(u_{4}\right)$ & $(3,[0.96,1.36])$ \\
\hline $\mathbb{C}\left(k_{1}, k_{4}\right)\left(u_{2}\right)$ & $(3,[0.83,1.53])$ & $\mathbb{C}\left(k_{3}, k_{5}\right)\left(u_{5}\right)$ & $(2,[1.30,1.66])$ \\
\hline $\mathbb{C}\left(k_{1}, k_{4}\right)\left(u_{3}\right)$ & $(0,[0.60,0.90])$ & $\mathbb{C}\left(k_{3}, k_{5}\right)\left(u_{6}\right)$ & $(3,[0.53,1.03])$ \\
\hline $\mathbb{C}\left(k_{1}, k_{4}\right)\left(u_{4}\right)$ & $(2,[0.50,1.03])$ & $\mathbb{C}\left(k_{3}, k_{7}\right)\left(u_{1}\right)$ & $(0,[1.03,1.46])$ \\
\hline $\mathbb{C}\left(k_{1}, k_{4}\right)\left(u_{5}\right)$ & $(4,[0.83,1.63])$ & $\mathbb{C}\left(k_{3}, k_{7}\right)\left(u_{2}\right)$ & $(4,[1.00,1.60])$ \\
\hline $\mathbb{C}\left(k_{1}, k_{4}\right)\left(u_{6}\right)$ & $(2,[0.76,1.40])$ & $\mathbb{C}\left(k_{3}, k_{7}\right)\left(u_{3}\right)$ & $(1,[0.86,1.76])$ \\
\hline $\mathbb{C}\left(k_{1}, k_{5}\right)\left(u_{1}\right)$ & $(1,[0.93,1.33])$ & $\mathbb{C}\left(k_{3}, k_{7}\right)\left(u_{4}\right)$ & $(3,[0.56,1.16])$ \\
\hline $\mathbb{C}\left(k_{1}, k_{5}\right)\left(u_{2}\right)$ & $(1,[0.56,1.56])$ & $\mathbb{C}\left(k_{3}, k_{7}\right)\left(u_{5}\right)$ & $(2,[0.96,1.33])$ \\
\hline $\mathbb{C}\left(k_{1}, k_{5}\right)\left(u_{3}\right)$ & $(0,[0.40,0.76])$ & $\mathbb{C}\left(k_{3}, k_{7}\right)\left(u_{6}\right)$ & $(4,[0.76,1.16])$ \\
\hline $\mathbb{C}\left(k_{1}, k_{5}\right)\left(u_{4}\right)$ & $(2,[0.93,1.30])$ & $\mathbb{C}\left(k_{5}, k_{4}\right)\left(u_{1}\right)$ & $(4,[0.50,1.40])$ \\
\hline $\mathbb{C}\left(k_{1}, k_{5}\right)\left(u_{5}\right)$ & $(4,[1.10,1.70])$ & $\mathbb{C}\left(k_{5}, k_{4}\right)\left(u_{2}\right)$ & $(3,[0.83,1.60])$ \\
\hline $\mathbb{C}\left(k_{1}, k_{5}\right)\left(u_{6}\right)$ & $(3,[0.76,1.23])$ & $\mathbb{C}\left(k_{5}, k_{4}\right)\left(u_{3}\right)$ & $(2,[1.06,1.46])$ \\
\hline $\mathbb{C}\left(k_{1}, k_{7}\right)\left(u_{1}\right)$ & $(1,[1.06,1.36])$ & $\mathbb{C}\left(k_{5}, k_{4}\right)\left(u_{4}\right)$ & $(3,[0.90,1.26])$ \\
\hline $\mathbb{C}\left(k_{1}, k_{7}\right)\left(u_{2}\right)$ & $(3,[0.83,1.56])$ & $\mathbb{C}\left(k_{5}, k_{4}\right)\left(u_{5}\right)$ & $(0,[0.73,1.46])$ \\
\hline $\mathbb{C}\left(k_{1}, k_{7}\right)\left(u_{3}\right)$ & $(0,[0.20,1.36])$ & $\mathbb{C}\left(k_{5}, k_{4}\right)\left(u_{6}\right)$ & $(1,[0.96,1.66])$ \\
\hline $\mathbb{C}\left(k_{1}, k_{7}\right)\left(u_{4}\right)$ & $(2,[0.50,1.10])$ & $\mathbb{C}\left(k_{5}, k_{5}\right)\left(u_{1}\right)$ & $(2,[0.76,1.26])$ \\
\hline $\mathbb{C}\left(k_{1}, k_{7}\right)\left(u_{5}\right)$ & $(2,[0.70,1.33])$ & $\mathbb{C}\left(k_{5}, k_{5}\right)\left(u_{2}\right)$ & $(1,[0.56,1.66])$ \\
\hline $\mathbb{C}\left(k_{1}, k_{7}\right)\left(u_{6}\right)$ & $(3,[0.96,1.30])$ & $\mathbb{C}\left(k_{5}, k_{5}\right)\left(u_{3}\right)$ & $(0,[0.96,1.40])$ \\
\hline $\mathbb{C}\left(k_{3}, k_{4}\right)\left(u_{1}\right)$ & $(0,[0.60,1.56])$ & $\mathbb{C}\left(k_{5}, k_{5}\right)\left(u_{4}\right)$ & $(3,[1.20,1.46])$ \\
\hline $\mathbb{C}\left(k_{3}, k_{4}\right)\left(u_{2}\right)$ & $(4,[0.96,1.53])$ & $\mathbb{C}\left(k_{5}, k_{5}\right)\left(u_{5}\right)$ & $(0,[1.00,1.50])$ \\
\hline $\mathbb{C}\left(k_{3}, k_{4}\right)\left(u_{3}\right)$ & $(1,[1.10,1.53])$ & $\mathbb{C}\left(k_{5}, k_{5}\right)\left(u_{6}\right)$ & $(1,[0.96,1.56])$ \\
\hline $\mathbb{C}\left(k_{3}, k_{4}\right)\left(u_{4}\right)$ & $(3,[0.56,1.10])$ & $\mathbb{C}\left(k_{5}, k_{7}\right)\left(u_{1}\right)$ & $(4,[0.96,1.33])$ \\
\hline $\mathbb{C}\left(k_{3}, k_{4}\right)\left(u_{5}\right)$ & $(2,[1.06,1.70])$ & $\mathbb{C}\left(k_{5}, k_{7}\right)\left(u_{2}\right)$ & $(3,[0.83,1.63])$ \\
\hline $\mathbb{C}\left(k_{3}, k_{4}\right)\left(u_{6}\right)$ & $(2,[0.53,1.23])$ & $\mathbb{C}\left(k_{5}, k_{7}\right)\left(u_{3}\right)$ & $(2,[0.86,1.70])$ \\
\hline $\mathbb{C}\left(k_{3}, k_{5}\right)\left(u_{1}\right)$ & $(0,[0.86,1.43])$ & $\mathbb{C}\left(k_{5}, k_{7}\right)\left(u_{4}\right)$ & $(3,[0.90,1.36])$ \\
\hline $\mathbb{C}\left(k_{3}, k_{5}\right)\left(u_{2}\right)$ & $(1,[0.80,1.63])$ & $\mathbb{C}\left(k_{5}, k_{7}\right)\left(u_{5}\right)$ & $(0,[0.53,1.03])$ \\
\hline $\mathbb{C}\left(k_{3}, k_{5}\right)\left(u_{3}\right)$ & $(0,[0.96,1.46])$ & $\mathbb{C}\left(k_{5}, k_{7}\right)\left(u_{6}\right)$ & $(1,[1.10,1.63])$ \\
\hline
\end{tabular}

Now we will evaluate the Score $\mathbb{S}\left(k_{s^{\prime}}, l_{t^{\prime}}\right)\left(u_{i}\right) ; \forall u_{i} \in U,\left(k_{s^{\prime}}, l_{t^{\prime}}\right) \in(K \times L), s^{\prime}=$ $1,3,5$ and $t^{\prime}=4,5,7$ by using step 4 of Algorithm 2 .

\begin{tabular}{lccc}
\hline $\mathbb{S}\left(k_{s^{\prime}}, k_{t^{\prime}}\right)\left(u_{i}\right)$ & The Score & $\mathbb{S}\left(k_{s^{\prime}}, k_{t^{\prime}}\right)\left(u_{i}\right)$ & The Score \\
\hline $\mathbb{S}\left(k_{1}, k_{4}\right)\left(u_{1}\right)$ & 7.67 & $\mathbb{S}\left(k_{3}, k_{5}\right)\left(u_{4}\right)$ & -1.08 \\
\hline $\mathbb{S}\left(k_{1}, k_{4}\right)\left(u_{2}\right)$ & 38.57 & $\mathbb{S}\left(k_{3}, k_{5}\right)\left(u_{5}\right)$ & 52.92 \\
\hline $\mathbb{S}\left(k_{1}, k_{4}\right)\left(u_{3}\right)$ & -37.56 & $\mathbb{S}\left(k_{3}, k_{5}\right)\left(u_{6}\right)$ & -83.16 \\
\hline $\mathbb{S}\left(k_{1}, k_{4}\right)\left(u_{4}\right)$ & -44.25 & $\mathbb{S}\left(k_{3}, k_{7}\right)\left(u_{1}\right)$ & 18.2 \\
\hline
\end{tabular}




\begin{tabular}{|c|c|c|c|}
\hline $\mathbb{S}\left(k_{s^{\prime}}, k_{t^{\prime}}\right)\left(u_{i}\right)$ & The Score & $\mathbb{S}\left(k_{s^{\prime}}, k_{t^{\prime}}\right)\left(u_{i}\right)$ & The Score \\
\hline $\mathbb{S}\left(k_{1}, k_{4}\right)\left(u_{5}\right)$ & 63.12 & $\mathbb{S}\left(k_{3}, k_{7}\right)\left(u_{2}\right)$ & 47.04 \\
\hline $\mathbb{S}\left(k_{1}, k_{4}\right)\left(u_{6}\right)$ & 12.45 & $\mathbb{S}\left(k_{3}, k_{7}\right)\left(u_{3}\right)$ & 31.2 \\
\hline $\mathbb{S}\left(k_{1}, k_{5}\right)\left(u_{1}\right)$ & 12 & $\mathbb{S}\left(k_{3}, k_{7}\right)\left(u_{4}\right)$ & -66.4 \\
\hline $\mathbb{S}\left(k_{1}, k_{5}\right)\left(u_{2}\right)$ & 1.92 & $\mathbb{S}\left(k_{3}, k_{7}\right)\left(u_{5}\right)$ & 1.7 \\
\hline $\mathbb{S}\left(k_{1}, k_{5}\right)\left(u_{3}\right)$ & -61.6 & $\mathbb{S}\left(k_{3}, k_{7}\right)\left(u_{6}\right)$ & -50.88 \\
\hline $\mathbb{S}\left(k_{1}, k_{5}\right)\left(u_{4}\right)$ & 12.3 & $\mathbb{S}\left(k_{5}, k_{4}\right)\left(u_{1}\right)$ & -58.08 \\
\hline $\mathbb{S}\left(k_{1}, k_{5}\right)\left(u_{5}\right)$ & 101.76 & $\mathbb{S}\left(k_{5}, k_{4}\right)\left(u_{2}\right)$ & 14.44 \\
\hline $\mathbb{S}\left(k_{1}, k_{5}\right)\left(u_{6}\right)$ & -11.78 & $\mathbb{S}\left(k_{5}, k_{4}\right)\left(u_{3}\right)$ & 20.8 \\
\hline $\mathbb{S}\left(k_{1}, k_{7}\right)\left(u_{1}\right)$ & 27.12 & $\mathbb{S}\left(k_{5}, k_{4}\right)\left(u_{4}\right)$ & -16.34 \\
\hline $\mathbb{S}\left(k_{1}, k_{7}\right)\left(u_{2}\right)$ & 37.44 & $\mathbb{S}\left(k_{5}, k_{4}\right)\left(u_{5}\right)$ & -8.84 \\
\hline $\mathbb{S}\left(k_{1}, k_{7}\right)\left(u_{3}\right)$ & -31.9 & $\mathbb{S}\left(k_{5}, k_{4}\right)\left(u_{6}\right)$ & 26.6 \\
\hline $\mathbb{S}\left(k_{1}, k_{7}\right)\left(u_{4}\right)$ & -37.24 & $\mathbb{S}\left(k_{5}, k_{5}\right)\left(u_{1}\right)$ & -28.08 \\
\hline $\mathbb{S}\left(k_{1}, k_{7}\right)\left(u_{5}\right)$ & -1.12 & $\mathbb{S}\left(k_{5}, k_{5}\right)\left(u_{2}\right)$ & -8.64 \\
\hline $\mathbb{S}\left(k_{1}, k_{7}\right)\left(u_{6}\right)$ & 23.4 & $\mathbb{S}\left(k_{5}, k_{5}\right)\left(u_{3}\right)$ & -0.84 \\
\hline $\mathbb{S}\left(k_{3}, k_{4}\right)\left(u_{1}\right)$ & -6 & $\mathbb{S}\left(k_{5}, k_{5}\right)\left(u_{4}\right)$ & 30.24 \\
\hline $\mathbb{S}\left(k_{3}, k_{4}\right)\left(u_{2}\right)$ & 35.52 & $\mathbb{S}\left(k_{5}, k_{5}\right)\left(u_{5}\right)$ & 5.04 \\
\hline $\mathbb{S}\left(k_{3}, k_{4}\right)\left(u_{3}\right)$ & 30.16 & $\mathbb{S}\left(k_{5}, k_{5}\right)\left(u_{6}\right)$ & 7.56 \\
\hline $\mathbb{S}\left(k_{3}, k_{4}\right)\left(u_{4}\right)$ & -66.5 & $\mathbb{S}\left(k_{5}, k_{7}\right)\left(u_{1}\right)$ & -2.88 \\
\hline $\mathbb{S}\left(k_{3}, k_{4}\right)\left(u_{5}\right)$ & 46.5 & $\mathbb{S}\left(k_{5}, k_{7}\right)\left(u_{2}\right)$ & 17.1 \\
\hline $\mathbb{S}\left(k_{3}, k_{4}\right)\left(u_{6}\right)$ & -43.5 & $\mathbb{S}\left(k_{5}, k_{7}\right)\left(u_{3}\right)$ & 24 \\
\hline $\mathbb{S}\left(k_{3}, k_{5}\right)\left(u_{1}\right)$ & -2.16 & $\mathbb{S}\left(k_{5}, k_{7}\right)\left(u_{4}\right)$ & -5.7 \\
\hline $\mathbb{S}\left(k_{3}, k_{5}\right)\left(u_{2}\right)$ & 6.6 & $\mathbb{S}\left(k_{5}, k_{7}\right)\left(u_{5}\right)$ & -58.5 \\
\hline $\mathbb{S}\left(k_{3}, k_{5}\right)\left(u_{3}\right)$ & 4.86 & $\mathbb{S}\left(k_{5}, k_{7}\right)\left(u_{6}\right)$ & 35.28 \\
\hline
\end{tabular}

By using step 5 of Algorithm 2 the weighted values for each $\mathbb{S}\left(u_{l}\right)$ is:

$$
\begin{gathered}
\mathbb{S}\left(u_{1}\right)=-32.21, \mathbb{S}\left(u_{2}\right)=189.99, \mathbb{S}\left(u_{3}\right)=-20.88, \\
\mathbb{S}\left(u_{4}\right)=-194.97, \mathbb{S}\left(u_{5}\right)=202.58, \mathbb{S}\left(u_{6}\right)=-84.03 .
\end{gathered}
$$

Here the optimal decision by using step 6 of Algorithm 2 is:

$$
\mathfrak{X}=\arg \max \left\{\mathbb{S}\left(u_{1}\right), \mathbb{S}\left(u_{2}\right), \mathbb{S}\left(u_{3}\right), \mathbb{S}\left(u_{4}\right), \mathbb{S}\left(u_{5}\right), \mathbb{S}\left(u_{6}\right)\right\} .
$$

Thus, $u_{5}$ is the best choice.

Hence $M r$. X will select $u_{5}$ person as a personal secretary.

In Figure 8, we observe the following relations between the score values of Examples 13 and 14 by means of Algorithms 1 and 2, respectively.

$$
\begin{aligned}
& \mathbb{S}\left(u_{4}\right) \preceq \mathbb{S}\left(u_{3}\right) \preceq \mathbb{S}\left(u_{6}\right) \preceq \mathbb{S}\left(u_{1}\right) \preceq \mathbb{S}\left(u_{5}\right) \preceq \mathbb{S}\left(u_{2}\right), \\
& \mathbb{S}\left(u_{4}\right) \preceq \mathbb{S}\left(u_{6}\right) \preceq \mathbb{S}\left(u_{1}\right) \preceq \mathbb{S}\left(u_{3}\right) \preceq \mathbb{S}\left(u_{2}\right) \preceq \mathbb{S}\left(u_{5}\right) .
\end{aligned}
$$




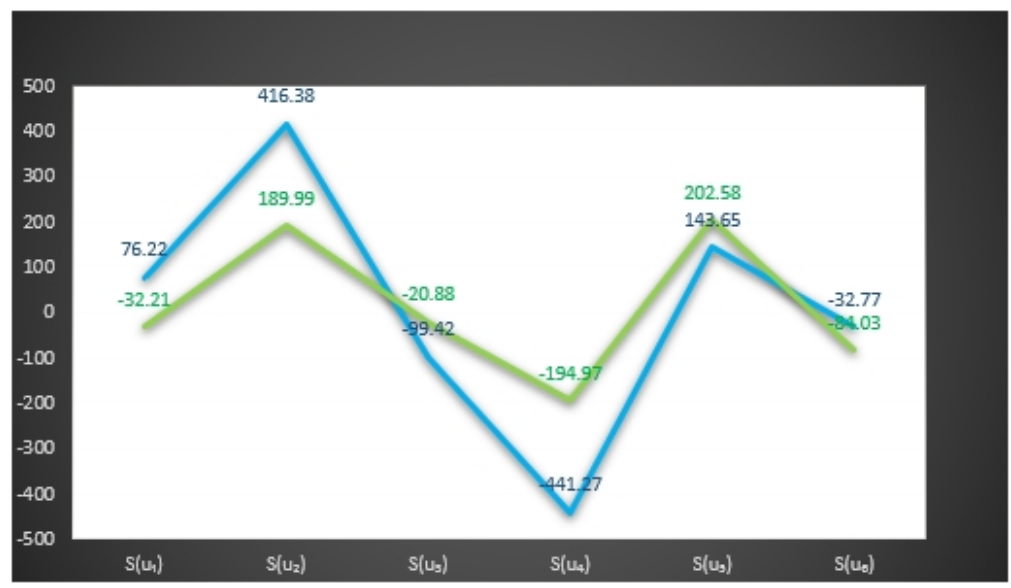

Figure 8. Comparison of score values of Examples 13 and 14 by means of Algorithms 2 and 2.

\section{Conclusions}

The main intent of this work is to present a possibility belief interval-valued N-soft set by consolidating a belief interval value and possibility with $\mathrm{N}$-soft set and its applications for solving the complicated decision-making problems in various fields of life. There are great applications for the belief interval in many different fields of life, while the other tool N-soft set theory is arising as a prosperous mathematical approach for manipulating ambivalent information. First, we defined basic theory and definitions of important sets in a very clear way. Then we discussed the BIVNSS, various algebraic operations, and their fundamental properties. Then we defined PBIVNSS, its algebraic operations, its elemental properties, and also its applications for decision-making problems. We have also provided algorithms for these decision-making methods and showed how decision-making methods are applied successfully in the problems of real life. In further work, this idea can be seen in many other algebraic expressions and topological structures.

Author Contributions: Conceptualization, S.A. and D.P.; methodology, Q.X.; software, M.S.H.; validation, Q.X. and S.A.; formal analysis, S.A.; investigation, M.K.; resources, M.S.H.; data curation, D.P.; writing-original draft preparation, Q.X.; writing—review and editing, R.F.; visualization, R.F.; supervision, S.A.; project administration, R.F. All authors have read and agreed to the published version of the manuscript.

Funding: This research received no external funding.

Data Availability Statement: No data were used to support this study.

Conflicts of Interest: The authors declare no conflict of interest.

\section{References}

1. Xiao, F.; Zhang, Z.; Abawajy, J. Workflow scheduling in distributed systems under fuzzy environment. J. Intell. Fuzzy Syst. 2019, 37, 5323-5333. [CrossRef]

2. Cao, Z.; Lin, C.T.; Lai, K.L.; Ko, L.W.; King, J.T.; Liao, K.K.; Fuh, J.L.; Wang, S.J. Extraction of SSVEPs-based inherent fuzzy entropy using a wearable headband EEG in migraine patients. IEEE Trans. Fuzzy Syst. 2019, 28, 14-27. [CrossRef]

3. Liu, F.; Gao, X.; Zhao, J.; Deng, Y. Generalized belief entropy and its application in identifying conflict evidence. IEEE Access 2019, 7, 126625-126633. [CrossRef]

4. Seiti, H.; Hafezalkotob, A.; Najafi, S.E.; Khalaj, M. A risk-based fuzzy evidential framework for FMEA analysis under uncertainty: An interval-valued DS approach. J. Intell. Fuzzy Syst. 2018, 35, 1419-1430. [CrossRef]

5. Wang, Q.; Li, Y.; Liu, X. Analysis of feature fatigue EEG signals based on wavelet entropy. Int. J. Pattern Recognit. Artif. Intell. 2018, 32, 1854023. [CrossRef]

6. Jiang, W.; Cao, Y.; Deng, X. A novel Z-network model based on Bayesian network and Z-number. IEEE Trans. Fuzzy Syst. 2019, 28, 1585-1599. [CrossRef]

7. Fu, C.; Chang, W.; Xue, M.; Yang, S. Multiple criteria group decision making with belief distributions and distributed preference relations. Eur. J. Oper. Res. 2019, 273, 623-633. [CrossRef]

8. Gao, X.; Deng, Y. Quantum model of mass function. Int. J. Intell. Syst. 2020, 35, 267-282. [CrossRef] 
9. Luo, Z.; Deng, Y. A vector and geometry interpretation of basic probability assignment in Dempster-Shafer theory. Int. J. Intell. Syst. 2020, 35, 944-962. [CrossRef]

10. Zadeh, L.A. Information and control. Fuzzy Sets 1965, 8, 338-353.

11. Fei, L. On interval-valued fuzzy decision-making using soft likelihood functions. Int. J. Intell. Syst. 2019, 34, 1631-1652. [CrossRef]

12. Song, Y.; Wang, X.; Quan, W.; Huang, W. A new approach to construct similarity measure for intuitionistic fuzzy sets. Soft Comput. 2019, 23, 1985-1998. [CrossRef]

13. Xue, Y.; Deng, Y. Entailment for intuitionistic fuzzy sets based on generalized belief structures. Int. J. Intell. Syst. 2020, 35, 963-982. [CrossRef]

14. Zhou, D.; Al-Durra, A.; Zhang, K.; Ravey, A.; Gao, F. A robust prognostic indicator for renewable energy technologies: A novel error correction grey prediction model. IEEE Trans. Ind. Electron. 2019, 66, 9312-9325. [CrossRef]

15. Molodtsov, D. Soft set theory-First results. Comput. Math. Appl. 1999, 37, 19-31. [CrossRef]

16. Feng, F.; Cho, J.; Pedrycz, W.; Fujita, H.; Herawan, T. Soft set based association rule mining. Knowl.-Based Syst. 2016, 111, 268-282. [CrossRef]

17. Feng, F.; Li, Y. Soft subsets and soft product operations. Inf. Sci. 2013, 232, 44-57. [CrossRef]

18. Xu, W.; Ma, J.; Wang, S.; Hao, G. Vague soft sets and their properties. Comput. Math. Appl. 2010, 59, 787-794. [CrossRef]

19. Maji, P.K.; Biswas, R.; Roy, A.R. Soft set theory. Comput. Math. Appl. 2003, 45, 555-562. [CrossRef]

20. Majumdar, P.; Samanta, S.K. Generalised fuzzy soft sets. Comput. Math. Appl. 2010, 59, 1425-1432. [CrossRef]

21. Maji, P.K.; Biswas, R.; Roy, A.R. Intuitionistic fuzzy soft sets. J. Fuzzy Math. 2001, 9, 677-692.

22. Maji, P.K. More on intuitionistic fuzzy soft sets. In International Workshop on Rough Sets, Fuzzy Sets, Data Mining, and Granular-Soft Computing; Springer: Berlin/Heidelberg, Germany, 2009; pp. 231-240.

23. Dinda, B.; Bera, T.; Samanta, T.K. Generalised intuitionistic fuzzy soft sets and its application in decision making. arXiv 2010, arXiv:1010.2468.

24. Dempster, A.P. Upper and lower probabilities induced by a multivalued mapping. In Classic Works of the Dempster-Shafer Theory of Belief Functions; Springer: Berlin/Heidelberg, Germany, 2008; pp. 57-72.

25. Cheng, C.; Cao, Z.; Xiao, F. A generalized belief interval-valued soft set with applications in decision making. Soft Comput. 2020, 24, 9339-9350. [CrossRef]

26. Jiang, Y.; Tang, Y.; Chen, Q.; Liu, H.; Tang, J. Interval-valued intuitionistic fuzzy soft sets and their properties. Comput. Math. Appl. 2010, 60, 906-918. [CrossRef]

27. Khalil, A.M.; Li, S.G.; Garg, H.; Li, H.; Ma, S. New operations on interval-valued picture fuzzy set, interval-valued picture fuzzy soft set and their applications. IEEE Access 2019, 7, 51236-51253. [CrossRef]

28. Deli, I. Interval-valued neutrosophic soft sets and its decision making. Int. J. Mach. Learn. Cybern. 2017, 8, 665-676. [CrossRef]

29. Khan, M.J.; Kumam, P.; Ashraf, S.; Kumam, W. Generalized picture fuzzy soft sets and their application in decision support systems. Symmetry 2019, 11, 415. [CrossRef]

30. Alkhazaleh, S.; Salleh, A.R.; Hassan, N. Possibility fuzzy soft set. Adv. Decis. Sci. 2011, 2011, 479756. [CrossRef]

31. Khalil, A.M.; Li, S.G.; Li, H.X.; Ma, S.Q. Possibility m-polar fuzzy soft sets and its application in decision-making problems. J. Intell. Fuzzy Syst. 2019, 37, 929-940. [CrossRef]

32. Jia-hua, D.; Zhang, H.; He, Y. Possibility Pythagorean fuzzy soft set and its application. J. Intell. Fuzzy Syst. 2019, 36, 413-421. [CrossRef]

33. Karaaslan, F. Possibility neutrosophic soft sets and PNS-decision making method. Appl. Soft Comput. 2017, 54, 403-414. [CrossRef]

34. Zhang, H.D.; Shu, L. Possibility multi-fuzzy soft set and its application in decision making. J. Intell. Fuzzy Syst. 2014, 27, 2115-2125. [CrossRef]

35. Shafer, G. A Mathematical Theory of Evidence; Princeton University Press: Princeton, NJ, USA, 1976; Volume 42.

36. Shenoy, P.P. Using Dempster-Shafer's belief-function theory in expert systems. In Applications of Artificial Intelligence X: KnowledgeBased Systems; International Society for Optics and Photonics: Bellingham, WA, USA, March 1992; Volume 1707, pp. 2-14.

37. Huang, Z.; Yang, L.; Jiang, W. Uncertainty measurement with belief entropy on the interference effect in the quantum-like Bayesian Networks. Appl. Math. Comput. 2019, 347, 417-428. [CrossRef]

38. Zhou, M.; Liu, X.B.; Chen, Y.W.; Yang, J.B. Evidential reasoning rule for MADM with both weights and reliabilities in group decision making. Knowl.-Based Syst. 2018, 143, 142-161. [CrossRef]

39. Zhou, M.; Liu, X.B.; Yang, J.B.; Chen, Y.W.; Wu, J. Evidential reasoning approach with multiple kinds of attributes and entropybased weight assignment. Knowl.-Based Syst. 2019, 163, 358-375. [CrossRef]

40. Kang, B.; Zhang, P.; Gao, Z.; Chhipi-Shrestha, G.; Hewage, K.; Sadiq, R. Environmental assessment under uncertainty using Dempster-Shafer theory and Z-numbers. J. Ambient. Intell. Humaniz. Comput. 2020, 11, 2041-2060. [CrossRef]

41. He, Z.; Jiang, W. An evidential dynamical model to predict the interference effect of categorization on decision making results. Knowl.-Based Syst. 2018, 150, 139-149. [CrossRef]

42. Zhou, M.; Liu, X.; Yang, J. Evidential reasoning approach for MADM based on incomplete interval value. J. Intell. Fuzzy Syst. 2017, 33, 3707-3721. [CrossRef]

43. Liu, Z.G.; Pan, Q.; Dezert, J.; Martin, A. Combination of classifiers with optimal weight based on evidential reasoning. IEEE Trans. Fuzzy Syst. 2017, 26, 1217-1230. [CrossRef] 
44. Pan, L.; Deng, Y. An association coefficient of a belief function and its application in a target recognition system. Int. J. Intell. Syst. 2020, 35, 85-104. [CrossRef]

45. Fatimah, F.; Rosadi, D.; Hakim, R.F.; Alcantud, J.C.R. N-soft sets and their decision making algorithms. Soft Comput. 2018, 22, 3829-3842. [CrossRef]

46. Akram, M.; Adeel, A.; Alcantud, J.C.R. Group decision-making methods based on hesitant N-soft sets. Expert Syst. Appl. 2019, 115, 95-105. [CrossRef]

47. Akram, M.; Adeel, A.; Alcantud, J.C.R. Fuzzy N-soft sets: A novel model with applications. J. Intell. Fuzzy Syst. 2018, 35, 4757-4771. [CrossRef]

48. Zeng, S.; Shoaib, M.; Ali, S.; Smarache, F.; Rashmanlou, H.; Mofidnakhaei, F. Certain properties of singlevalued neutrosophic graph with application in food and agriculture organization. Int. J. Comput. Intell. Syst. 2021, 14, 1516-1540. [CrossRef]

49. Gulfam, M.; Mahmood, M.K.; Smarandache, F.; Ali, S. New Dombi aggregation operators on bipolar neutrosophic set with application in multi-attribute decision-making problems. J. Intell. Fuzzy Syst. 2021, 40, 5043-5060. [CrossRef]

50. Hameed, M.S.; Mukhtar, S.; Khan, H.N.; Ali, S.; Mateen, M.H.; Gulzar, M. Pythagorean fuzzy N-Soft groups. Int. J. Electr. Comput. Eng. 2021, 21, 1030-1038.

51. Liu, J.B.; Ali, S.; Mahmood, M.K.; Mateen, M.H. On m-polar diophantine fuzzy Nsoft set with applications. Comb. Chem. High Throughput Screen. 2020, 23. [CrossRef] [PubMed]

52. Zeng, S.; Shoaib, M.; Ali, S.; Abbas, Q.; Nadeem, M.S. Complex vague graphs and their application in decision-making problems. IEEE Access 2020, 8, 174094-174104. [CrossRef]

53. Mahmood, M.K.; Zeng, S.; Gulfam, M.; Ali, S.; Jin, Y. Bipolar neutrosophic dombi aggregation operators with application in multi-attribute decision making problems. IEEE Access 2020, 8, 156600-156614. [CrossRef]

54. Akram, M.; Amjad, U.; Davvaz, B. Decision-making analysis based on bipolar fuzzy N-soft information. Comput. Appl. Math 2021, 40, 182. [CrossRef]

55. Akram, M.; Luqman, A.; Kahraman, C.Hesitant Pythagorean fuzzy ELECTRE-II method for multi-criteria decision-making problems. Appl. Soft Comput. 2021, 108, 107479. [CrossRef]

56. Adeel, A.; Akram, M.; Cagman, N. Decision-making analysis based on hesitant fuzzy N-Soft ELECTRE-I approach. Soft Comput. 2021. [CrossRef]

57. Akram, M.; Kahraman, C.; Zahid, K. Extension of TOPSIS model to the decision-making under complex spherical fuzzy information. Soft Comput. 2021, 25, 10771-10795. [CrossRef]

58. Akram, M.; Alsulami, S.; Zahid, K. A hybrid method for complex pythagorean fuzzy decision making. Math. Probl. Eng. 2021, 2021, 9915432. [CrossRef]

59. Liu, Y.T.; Pal, N.R.; Marathe, A.R.; Lin, C.T. Weighted fuzzy Dempster-Shafer framework for multimodal information integration. IEEE Trans. Fuzzy Syst. 2017, 26, 338-352. [CrossRef]

60. Gong, Y.; Su, X.; Qian, H.; Yang, N. Research on fault diagnosis methods for the reactor coolant system of nuclear power plant based on DS evidence theory. Ann. Nucl. Energy 2018, 112, 395-399. [CrossRef]

61. Mo, H.; Deng, Y. Identifying node importance based on evidence theory in complex networks. Phys. A Stat. Mech. Its Appl. 2019, 529, 121538. [CrossRef]

62. Seiti, H.; Hafezalkotob, A. Developing pessimistic-optimistic risk-based methods for multi-sensor fusion: An interval-valued evidence theory approach. Appl. Soft Comput. 2018, 72, 609-623. [CrossRef]

63. Deng, X.; Jiang, W.; Wang, Z. Zero-sum polymatrix games with link uncertainty: A Dempster-Shafer theory solution. Appl. Math. Comput. 2019, 340, 101-112. [CrossRef]

64. Xiao, F. A new divergence measure for belief functions in D-S evidence theory for multisensor data fusion. Inf. Sci. 2020, 514, 462-483. [CrossRef]

65. Xiao, F. EFMCDM: Evidential fuzzy multicriteria decision making based on belief entropy. IEEE Trans. Fuzzy Syst. 2019, 28, 1477-1491. [CrossRef]

66. Cui, H.; Liu, Q.; Zhang, J.; Kang, B. An improved deng entropy and its application in pattern recognition. IEEE Access 2019, 7, 18284-18292. [CrossRef]

67. Ozkan, K. Comparing Shannon entropy with Deng entropy and improved Deng entropy for measuring biodiversity when a priori data is not clear. Forestist 2018, 68, 136-140.

68. Xiao, F. Generalization of Dempster-Shafer theory: A complex belief function. arXiv 2019, arXiv:1906.11409.

69. Jiang, W. A correlation coefficient for belief functions. Int. J. Approx. Reason. 2018, 103, 94-106. [CrossRef] 